\title{
The New Heavy Mesons: A Status Report
}

\author{
Eric S. Swanson* \\ Department of Physics and Astronomy, University of Pittsburgh \\ Pittsburgh, PA 15260, USA
}

\begin{abstract}
A survey of the experimental, phenomenological, and theoretical status of the new heavy mesons is presented. States discussed are the $B_{c}, h_{c}, \eta_{c}^{\prime}, D_{s}(2317), D_{s}(2460), X(3872), X(3940), Y(3940)$, $Z(3930)$, and $Y(4260)$. Quark models for spectra, strong decays, and hadronic interactions are reviewed and used to interpret the new states. New results for strong decay models, bound state decays, mesonic molecules, properties of the $\mathrm{X}(3872)$, and the chiral doublet model are also presented.
\end{abstract}

\section{Contents}

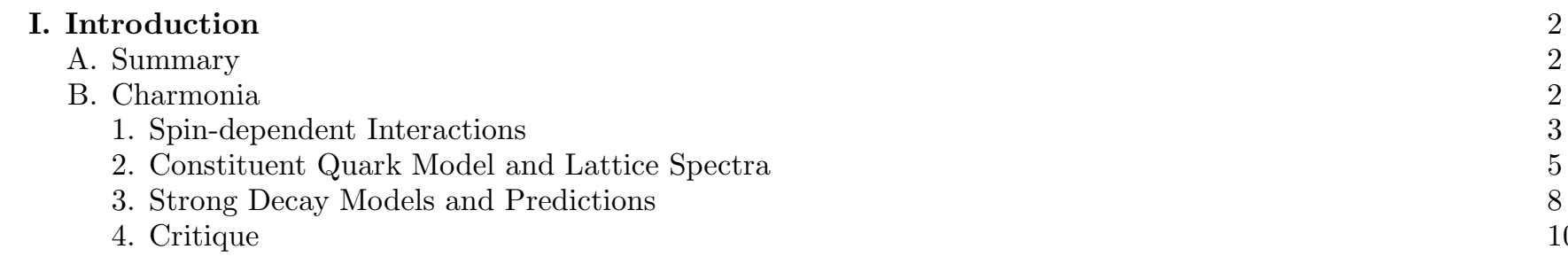

\begin{tabular}{|lr} 
II. The X(3872) & 12
\end{tabular}

A. Experiment 12

B. Models 15

1. Charmonium 15

2. Tetraquarks 16

3. Additional Models 17

4. Molecular Interpretation of the $X$

C. Effective Field Theorv 21

D. Microscopic Decav Models 24

1. Strong Decavs 24

2. Radiative Decavs 25

E. New Data and Further Analvsis 26

F. Summary 28

G. Other Molecules 28

III. Other New Charmonia 30

\begin{tabular}{ll} 
A. $h$ d & 30 \\
\hline
\end{tabular}

B. $\eta^{\prime} \quad 30$

C. $X(3940)$

D. $Y(3940)$

E. $Z(3930)$

F. $Y(4260)] 35$

IV. New Open Charm States $\quad 37$

A. Heavv Quark Svmmetrv and the Quark Model 237

1. Notation 37

2. Spin Splitting and Multiplet Structure $\quad 37$

3. Decavs $\quad 38$

4. Spin Splitting with Continuum Mixing 40

5. D-waves 41

* swansone@pitt.edu 
B. Chiral Svmmetrv Breaking and Restoration in Heavv-Light Mesons 42

1. Effective Field Theorv 42

2. An Explicit Chiral Doublet Model 43

C. $D_{s}(2317)$ and $D_{s}(2460)$

1. Experiment

2. Models 47

3. Summary 49

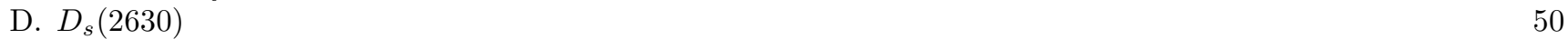

$\begin{array}{ll}\text { E. P-wave } B \text { Mesons } & 51\end{array}$

$\begin{array}{ll}\text { F. } B_{d} & 52\end{array}$

$\begin{array}{ll}\text { V. Conclusions } & 53\end{array}$

$\begin{array}{ll}\text { Acknowledgments } & 54\end{array}$

$\begin{array}{ll}\text { References } & 55\end{array}$

$\begin{array}{ll}\text { A. Bound State Decav Formalism } & 58\end{array}$

$\begin{array}{ll}\text { B. Pion Exchange Potentials } & 60\end{array}$

\section{INTRODUCTION}

More than 30 years after the November Revolution, charmonium spectroscopy continues to surprise and challenge. A new era began in April of 2003 when BaBar announced the discovery of the enigmatic $D_{s}(2317)$. Since then CLEO, Belle, Fermilab, and BES have joined the scrum, and the number of new states has risen to the double digits.

The formerly comfortable world of heavy quark spectroscopy is being severely tested by new experiment [1]. This report summarises the experimental status of eleven new open and hidden charm mesons ${ }^{1}$, the challenges these make to our understanding of strong QCD, and the theoretical attempts to meet these challenges.

This report begins with a summary of the new eleven and a review of what is expected in charmonium spectroscopy. For the latter, several models (new and old) are compared to the data, and three models of strong decays are examined. This serves to illustrate what is known (and not known) before examining the new charmonia states. A similar introduction to heavy-light systems is made in Sections IV.A and IV.B.

\section{A. Summary}

Table【summarises the contents of this report for the impatient. A selection of interpretations and theoretical ideas are listed in the 'comments' column. Theory and more experimental detail can be found in the main text.

\section{B. Charmonia}

This section describes the current status of charmonium and strong decay (mostly constituent quark and lattice gauge theory). This is in an effort to set the stage for the new hadrons to be described in subsequent sections and to establish the degree of reliability which we may attach to those expectations.

The utility of the nonrelativistic limit in describing charmonia has been recognised since the days of the November Revolution [2, 3]. The subsequent advent of the operator product [4], nonrelativistic [5], and potential nonrelativistic QCD [6] formalisms has considerably strengthened the connection of heavy quark phenomenology with $\mathrm{QCD}^{2}$. For example, the hierarchy of scales $m_{c}>>m_{c} \alpha_{s}\left(m_{c}\right)>>m_{c} \alpha_{s}^{2}\left(m_{c}\right)$ permits one to choose a cutoff which removes transverse gluons from the effective description of a meson. Thus an efficient potential description and Fock space expansion of charmonium is feasible 7 .

\footnotetext{
1 States not discussed here include the X(1835), putative glueballs, hybrids $\pi_{1}(1400), \pi_{1}(1600)$, and $\pi(1800)$, and baryons

${ }^{2}$ It also reveals that the charm quark is uncomfortably light.
} 
TABLE I: New Heavy Mesons

\begin{tabular}{|c|c|c|c|c|c|}
\hline state & mass $(\mathrm{MeV})$ & width $(\mathrm{MeV})$ & production/decay mode & comments & ref \\
\hline$h_{c}$ & $3524.4 \pm 0.6 \pm 0.4$ & - & 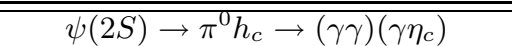 & ב $\approx$ CQM / tests spin dependence & $\overline{\mathrm{CLEO}[87]}$ \\
\hline$\eta_{c}^{\prime}$ & $3654 \pm 6 \pm 8$ & $<55$ & $B \rightarrow K \eta_{c}^{\prime} \rightarrow K K_{S} K \pi$ & $\approx$ CQM / tests hyperfine splitting & Belle[90] \\
\hline & $3642.9 \pm 3.1 \pm 1.5$ & $6.3 \pm 12.4 \pm 4.0$ & $e^{+} e^{-} \rightarrow \eta_{c}^{\prime} J / \psi$ & & CLEO [93] \\
\hline & $3630.8 \pm 3.4 \pm 1.0$ & $17.0 \pm 8.0 \pm 2.5$ & $\gamma \gamma \rightarrow \eta_{c}^{\prime} \rightarrow K_{S} K \pi$ & & $\mathrm{BaBar}[94]$ \\
\hline \multirow[t]{6}{*}{$X(3872)$} & $3872.0 \pm 0.6 \pm 0.5$ & $<2.395 \%$ C.L. & $B \rightarrow K X \rightarrow K \pi \pi J / \psi$ & molecule, cusp, tetraquark & Belle[32] \\
\hline & $3873.4 \pm 1.4$ & - & $B \rightarrow K X \rightarrow K \pi \pi J / \psi$ & & BaBar[35] \\
\hline & - & - & $B \rightarrow X \rightarrow \pi \pi \pi J / \psi$ & & Belle[43] \\
\hline & - & - & $B \rightarrow X \rightarrow \gamma J / \psi$ & & Belle[43] \\
\hline & $3871.3 \pm 0.7 \pm 0.4$ & - & $p \bar{p} \rightarrow X \rightarrow \pi \pi J / \psi$ & & $\mathrm{CDF}[33]$ \\
\hline & $3871.8 \pm 3.1 \pm 3.0$ & - & $p \bar{p} \rightarrow X \rightarrow \pi \pi J / \psi$ & & $\mathrm{D} \varnothing[34]$ \\
\hline$X(3940)$ & $\begin{array}{c}\text { avg }=3871.9 \pm 0.5 \\
3943 \pm 6 \pm 6\end{array}$ & $<52$ & $e^{+} e^{-} \rightarrow J / \psi X \rightarrow J / \psi D \bar{D}^{*}$ & $\chi_{c 1}^{\prime}, \eta_{c}^{\prime \prime} /$ needs confirm & Belle[96] \\
\hline$Y(3940)$ & $3943 \pm 11 \pm 13$ & $87 \pm 22 \pm 26$ & $B \rightarrow K Y \rightarrow K \pi \pi \pi J / \psi$ & needs confirmation & Belle[105] \\
\hline$Z(3930)$ & $3931 \pm 4 \pm 2$ & $20 \pm 8 \pm 3$ & $\gamma \gamma \rightarrow Z \rightarrow D \bar{D}$ & $\chi_{c 2}^{\prime} / \approx \mathrm{CQM}$ & Belle[107] \\
\hline$Y(4260)$ & $4259 \pm 8 \pm 4$ & $88 \pm 23 \pm 5$ & $e^{+} e^{-} \rightarrow \gamma_{I S R} Y \rightarrow \gamma_{I S R} J / \psi \pi \pi$ & hybrid?/ needs confirmation & $\mathrm{BaBar}[109]$ \\
\hline \multirow[t]{3}{*}{$\overline{D_{s}(2317)}$} & $2317.3 \pm 0.4 \pm 0.8$ & $<10$ & $e^{+} e^{-} \rightarrow D_{s}(2317) \rightarrow D_{s} \pi^{0}$ & molecule, tetraquark, shifted $c \bar{s}$ & $\overline{\mathrm{BaBar}}[121]$ \\
\hline & $2319.8 \pm 2.1 \pm 2.0$ & $\approx 0$ & $B \rightarrow \bar{D} D_{s}(2317) \rightarrow \bar{D} D_{s} \pi^{0}$ & & Belle[139] \\
\hline & $2318.5 \pm 1.2 \pm 1.1$ & & $D_{s}(2317) \rightarrow D_{s} \pi^{0}$ & & CLEO[132] \\
\hline \multirow[t]{3}{*}{$D_{s}(2460)$} & $2463.6 \pm 1.7 \pm 1.2$ & $<790 \%$ C.L. & $D_{s}(2460) \rightarrow D_{s}^{*} \pi^{0}$ & molecule, tetraquark, shifted $c \bar{s}$ & CLEO[132] \\
\hline & $2458.0 \pm 1.0 \pm 1.0$ & resolution & $D_{s}(2460) \rightarrow D_{s} \pi^{0} \gamma$ & & $\mathrm{BaBar}[134]$ \\
\hline & $2459.2 \pm 1.6 \pm 2.0$ & $\approx 0$ & $B \rightarrow \bar{D} D_{s}(2460) \rightarrow \bar{D} D_{s}^{*} \pi^{0}, \bar{D} D_{s} \gamma$ & & Belle[139] \\
\hline$D_{s}(2630)$ & $2632.6 \pm 1.6$ & $<1790 \%$ C.L. & $D_{s} \rightarrow D^{0} K^{+}$and $D_{s} \eta$ & artefact & SELEX $[168$ \\
\hline$B_{c}$ & $6285.7 \pm 5.3 \pm 1.2$ & $0.474 \pm 0.07 \pm 0.33 \mathrm{ps}$ & $p \bar{p} \rightarrow B_{c} \rightarrow J / \psi \pi^{ \pm}$ & $\approx \mathrm{CQM}$ & $\mathrm{CDF}[180]$ \\
\hline
\end{tabular}

\section{Spin-dependent Interactions}

Typical nonrelativistic quark models contain the following ingredients: nonrelativistic quark kinetic energy, a central confining potential, and a variety of spin-dependent $(v / c)^{2}$ corrections. Thus one has

$$
H_{C Q M}=K(q)+K(\bar{q})+V_{c o n f}+V_{S D}
$$

where $K$ is a quark kinetic energy, $V_{\text {conf }}$ is the central confining potential, and $V_{S D}$ is the spin-dependent potential.

The form of the spin-dependent interaction has been computed by Eichten and Feinberg $[\underline{8}$ at the tree level using the Wilson loop methodology. This result was extended by Pantaleone, Tye, and $\mathrm{Ng}[\underline{9}]$ in a one-loop computation. The net result is:

$$
\begin{aligned}
V_{S D}(r)= & \left(\frac{\boldsymbol{\sigma}_{q}}{4 m_{q}^{2}}+\frac{\boldsymbol{\sigma}_{\bar{q}}}{4 m_{\bar{q}}^{2}}\right) \cdot \mathbf{L}\left(\frac{1}{r} \frac{d \epsilon}{d r}+\frac{2}{r} \frac{d V_{1}}{d r}\right)+\left(\frac{\boldsymbol{\sigma}_{\bar{q}}+\boldsymbol{\sigma}_{q}}{2 m_{q} m_{\bar{q}}}\right) \cdot \mathbf{L}\left(\frac{1}{r} \frac{d V_{2}}{d r}\right) \\
& +\frac{1}{12 m_{q} m_{\bar{q}}}\left(3 \boldsymbol{\sigma}_{q} \cdot \hat{\mathbf{r}} \boldsymbol{\sigma}_{\bar{q}} \cdot \hat{\mathbf{r}}-\boldsymbol{\sigma}_{q} \cdot \boldsymbol{\sigma}_{\bar{q}}\right) V_{3}+\frac{1}{12 m_{q} m_{\bar{q}}} \boldsymbol{\sigma}_{q} \cdot \boldsymbol{\sigma}_{\bar{q}} V_{4} \\
& +\frac{1}{2}\left[\left(\frac{\boldsymbol{\sigma}_{q}}{m_{q}^{2}}-\frac{\boldsymbol{\sigma}_{\bar{q}}}{m_{\bar{q}}^{2}}\right) \cdot \mathbf{L}+\left(\frac{\boldsymbol{\sigma}_{q}-\boldsymbol{\sigma}_{\bar{q}}}{m_{q} m_{\bar{q}}}\right) \cdot \mathbf{L}\right] V_{5} .
\end{aligned}
$$

Here $\epsilon=\epsilon(r)$ is the static potential, $\mathbf{L}=\mathbf{L}_{q}=-\mathbf{L}_{\overline{\mathbf{q}}}, r=|\mathbf{r}|=\left|\mathbf{r}_{q}-\mathbf{r}_{\bar{q}}\right|$ is the $\bar{Q} Q$ separation and the $V_{i}=V_{i}\left(m_{q}, m_{\bar{q}} ; r\right)$ are determined by electric and magnetic field insertions on quark lines in the Wilson loop matrix element. $V_{5}$ vanishes in the equal quark mass case. As shown by Gromes [10] covariance under Lorentz transformation leads to a constraint between the SD potentials,

$$
\epsilon^{\prime}(r)=V_{2}^{\prime}(r)-V_{1}^{\prime}(r)
$$

In a model approach, $Q \bar{Q}$ interactions are typically derived from a nonrelativistic reduction of a relativistic currentcurrent interaction 


$$
H_{\text {int }}=\frac{1}{2} \int d^{3} x d^{3} y J_{\Gamma}(x) K(x-y) J_{\Gamma}(y)
$$

where $J_{\Gamma}=\bar{\psi} \Gamma \psi$ specifies a quark current.

As far as long range potentials are concerned only time-like vector or scalar currents are relevant since other currents do not yield static central potentials [12]. Vector confinement interactions are motivated as natural extensions of onegluon-exchange. More fundamentally, the instantaneous nonperturbative interaction of QCD in Coulomb gauge has the Lorentz structure $V^{0} \otimes V^{0}$. Scalar interactions are also often considered when model building for reasons to be discussed shortly.

Nonrelativistic reductions of vector or scalar interactions yield:

$$
\begin{array}{lll}
V_{1}=0, & V_{2}=\epsilon, \quad V_{3}=\epsilon^{\prime} / r-\epsilon^{\prime \prime}, \quad V_{4}=2 \nabla^{2} \epsilon \quad \text { vector } \\
V_{1}=-\epsilon, & V_{2}=V_{3}=V_{4}=0 \quad \text { scalar. } &
\end{array}
$$

Determining the Dirac structure of the long range potential yields important information on the nonperturbative gluodynamics which gives rise to confinement. This can be accomplished by examining spin-dependent mass splittings in quarkonia, in particular the ratio of P-wave splittings defined by

$$
\rho=\frac{2^{++}-1^{++}}{1^{++}-0^{++}}
$$

is a useful diagnostic [11].

Perturbative P-wave quarkonium splittings are given by

$$
\begin{aligned}
2^{++} & =E_{0}-\frac{2}{5} T+L+\frac{1}{4} S \\
1^{++} & =E_{0}+2 T-L+\frac{1}{4} S \\
0^{++} & =E_{0}-4 T-2 L+\frac{1}{4} S \\
1^{+-} & =E_{0}-\frac{3}{4} S
\end{aligned}
$$

where

$$
\begin{aligned}
T & =\frac{1}{12 m^{2}}\left\langle V_{3}\right\rangle \\
L & =\frac{1}{m^{2}}\left\langle\frac{\epsilon^{\prime}}{2 r}+\frac{V_{1}^{\prime}}{r}+\frac{V_{2}^{\prime}}{r}\right\rangle \\
S & =\frac{1}{3 m^{2}}\left\langle V_{4}\right\rangle .
\end{aligned}
$$

Using the results given in Eq. 5 gives vector and scalar spin splittings ratios of

$$
\rho(V)=\frac{\left\langle\frac{16}{5} \frac{\alpha_{s}}{r^{3}}+\frac{14}{5} \frac{b}{r}\right\rangle}{\left\langle 4 \frac{\alpha_{s}}{r^{3}}+2 \frac{b}{r}\right\rangle}
$$

and

$$
\rho(S)=\frac{\left\langle\frac{16}{5} \frac{\alpha_{s}}{r^{3}}-\frac{b}{r}\right\rangle}{\left\langle 4 \frac{\alpha_{s}}{r^{3}}-\frac{b}{2 r}\right\rangle} .
$$

The measured values for this parameter are $\rho\left(\chi_{c}\right)=0.48(1)$ and $\rho\left(\chi_{b}\right)=0.66(2)$. Notice that the results given here imply that $\rho(Q \bar{Q}) \rightarrow 4 / 5$ in the heavy quark limit. Thus, although the motion is in the right direction, even the $\chi_{b}$ system is not yet heavy by this metric. 
Fig. 1 displays the splitting ratio versus the RMS meson radius along with the experimentally measured ratios for the $\psi$ and $\Upsilon$ systems. It is clear that the vector confinement hypothesis cannot explain the data. Alternatively, the scalar hypothesis decreases with increasing RMS radius as required. Arrows in the figure show RMS radii for the $\chi_{c}$ and $\chi_{b}$ systems computed in a simple nonrelativistic model, indicating that scalar confinement is also quantitatively reliable.

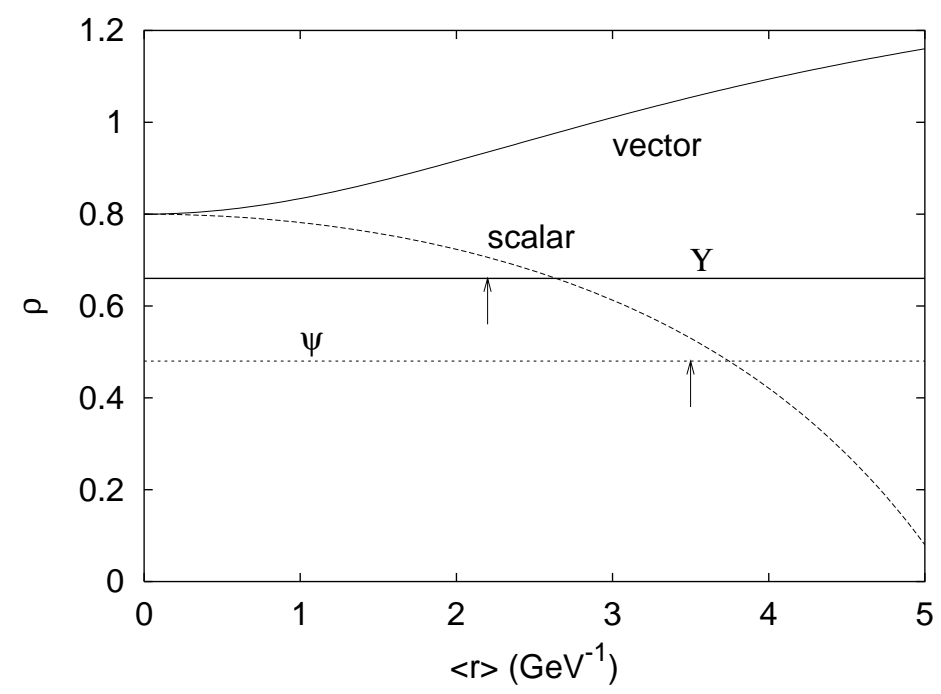

FIG. 1: Mass Splitting Ratio vs. $r_{R M S}$. Experimental values are given as horizontal lines.

The hyperfine interaction $V_{4}$ may be probed by comparing the mass splitting between the $\mathrm{P}$-wave system and the axial vector quarkonium state. Specifically, perturbative tensor and spin-orbit splittings are not present in the 'centre of gravity' of the $P$-wave system:

$$
M_{\text {cog }}=\frac{1}{9}\left(M\left(0^{++}\right)+3 M\left(1^{++}\right)+5 M\left(2^{++}\right)\right)=E_{0}+\frac{1}{4} S .
$$

Thus

$$
M_{c o g}-M\left(1^{+-}\right)=S \text {. }
$$

For one gluon exchange $S \propto\langle\delta(\vec{r})\rangle$ and since this expectation value is zero in P-waves, very little axial-cog mass splitting is expected (little because the delta function is smeared by zitterbewegung effects). Alternatively, for a vector confining potential $S=\frac{4 b}{3 m^{2}}\left\langle\frac{1}{r}\right\rangle$ and a nonzero splitting can be expected. The recent discovery of the $h_{c}$ permits this splitting to be measured. The results are discussed in Section IIIA

Simple spectroscopy teaches us about the $v^{2} / c^{2}$ structure of the confinement potential, and therefore something about the nonperturbative gluodynamics associated with flux tubes. This is nontrivial and worthy of pursuit!

\section{Constituent Quark Model and Lattice Spectra}

The predictions of four representative constituent quark models are given in Table III These are a simple nonrelativistic model[13], an updated version of the 'relativised' Godfrey-Isgur model[13], a 'relativistic' quark model[14], and the original Cornell model[3].

The simple constituent quark model (labelled BGS in Table III) employs a nonrelativistic quark kinetic energy, $K(q)=m_{q}-\nabla^{2} / 2 m_{q}$, a 'Coulomb+linear' central potential, $V_{\text {conf }}=-4 \alpha_{s} /(3 r)+b r$ and a spin-dependent interaction that corresponds to the vector one-gluon-exchange + scalar confinement Ansatz in the equal quark mass case:

$$
V_{1}=-b r, \quad V_{2}=-\frac{4}{3} \frac{\alpha_{s}}{r}, \quad V_{3}=4 \frac{\alpha_{s}}{r^{3}}, \quad V_{4}=\frac{32 \pi \alpha_{s}}{3} \tilde{\delta}_{\sigma}(r), \quad V_{5}=0
$$


where $\tilde{\delta}_{\sigma}(r)=(\sigma / \sqrt{\pi})^{3} e^{-\sigma^{2} r^{2}}$ represents a 'smeared' delta function. Fitting the charmonium spectrum yields: $\alpha_{s}=0.5462, b=0.1425 \mathrm{GeV}^{2}, m_{c}=1.4794 \mathrm{GeV}$, and $\sigma=1.0946 \mathrm{GeV}$.

The Godfrey-Isgur model is a "relativised" extension of typical nonrelativistic quark models. In particular, a relativistic dispersion relation for the quark kinetic energy $K(q)=\sqrt{m_{q}^{2}+\nabla^{2}}$, a QCD-motivated running coupling $\alpha_{s}(r)$, a flavor-dependent potential smearing parameter $\sigma \rightarrow \sigma\left(m_{q}, m_{\bar{q}}\right)$, and additional factors of $m_{q} / K(q)$ are all used [13, 18]. Just as in the nonrelativistic model, the quark-antiquark potential $\mathrm{V}_{q \bar{q}}(\vec{p}, \vec{r})$ incorporates the Lorentz vector one gluon exchange interaction at short distances and a Lorentz scalar linear confining interaction. Note that the string tension and quark mass $\left(b=0.18 \mathrm{GeV}^{2}\right.$ and $\left.m_{c}=1.628 \mathrm{GeV}\right)$ are significantly larger than the values used in the nonrelativistic model. Predictions of this model are in the column labelled GI in Table III

Ebert, Faustov, and Galkin[14] attempt a more formal ('quasipotential') approach to relativising the quark model. A nonrelativistic dispersion relation is used but with reduced and total masses determined relativistically (for example, the reduced mass is given by $\mu=E_{q} E_{\bar{q}} /\left(E_{q}+E_{\bar{q}}\right)$ with $E_{q}=\left(M^{2}-m_{\bar{q}}^{2}+m_{q}^{2}\right) /(2 M)$ and $M$ is the eigenenergy). The interaction employs the complete Coulomb gauge gluon propagator (so that the temporal and spatial vector interactions are included), a scalar confinement potential, and a vector confinement potential with an anomalous magnetic moment $\kappa$ for the quark. In the nonrelativistic limit, the confinement potential is $V_{\text {conf }}(r)=b r+B$ where the linear potential receives contributions from the vector and scalar confinement terms of $(1-\delta) b r+B$ and $\delta b r$ respectively where $\delta$ is a mixing coefficient. The coupling is fixed for each quark flavour and is $\alpha_{s}=0.314$ for charmonia. Additional parameters are determined to be $m_{c}=1.55 \mathrm{GeV}, b=0.18 \mathrm{GeV}^{2}, B=-0.16 \mathrm{GeV}, \kappa=-1$. Finally, at $\mathcal{O}\left(v^{2} / c^{2}\right)$ the spin-dependent interaction reduces to precisely that used in the BGS model discussed above (when $\kappa=-1$ ). For example, the long range contribution to $V_{4}$ is

$$
V_{4}(r)=4(1+\kappa)^{2}(1-\delta) \frac{b}{r}
$$

which is disfavoured by the data so that that this choice of the anomalous magnetic moment eliminates unwanted long range chromomagnetic interactions 19]. The predictions of this model are labelled EFG in Table II

The final model is that of the Cornell group [3], whose original predictions are reproduced in the sixth column of Table II The starting point is an assumed nonrelativistic kinetic energy and a quark-quark interaction mediated by

$$
H_{\text {int }}=\frac{1}{2} \int d^{3} x d^{3} y \rho^{a}(x) K(x-y) \rho^{a}(y)
$$

with the kernel given by $K(r)=\alpha_{s} / r-\frac{3}{4} b r$ and the current given by the colour charge density, $\rho^{a}=\psi^{\dagger} T^{a} \psi$. Of course this is a vector confinement model so that the nonrelativistic reduction of Eq. 20 corresponds to

$$
\epsilon(r)=-\frac{4}{3} K(r), \quad V_{1}=0, \quad V_{2}=-\frac{4}{3} K(r), \quad V_{3}=4 \frac{\alpha_{s}}{r^{3}}+\frac{b}{r}, \quad V_{4}=-8 \pi \alpha_{s} \delta(\vec{r})+4 \frac{b}{r}
$$

Fitted parameters are $m_{c}=1.84 \mathrm{GeV}, b=0.183 \mathrm{GeV}^{2}$, and $\alpha_{s}=0.39$. Spin-dependent effects are ignored in the original Cornell papers. However, the Cornell group does attempt to include the effects of open charm virtual states on the spectrum (this will be discussed in greater detail in Sections IB 3 and IB 4). The resulting masses are shown in square brackets in Table We note that the coupled channel approach shifts most of the predicted masses a modest amount, except the $\psi(3 S)$ and $\psi(4 S)$ states, for which the naive predictions have been considerably worsened.

The advent of QCD motivated quark models coincided with the invention of lattice gauge theory and the lattice has provided an important counterpoint to models ever since. Recently, lattice computations have become sufficiently robust that attempts have been made to compute extensive spectra. Two such computations are presented in the last columns of Table III These computations have been made in the 'quenched' approximation where the effects of virtual quarks are neglected on anisotropic lattices with a lattice volume fixed at $(1.6 \mathrm{fm})^{3}$ in Ref. 15] or over lattice volumes ranging from $(1.5 \mathrm{fm})^{3}$ to $(3.3 \mathrm{fm})^{3}$ in Ref. [16]. Continuum extrapolated values are quoted in the table.

Overall the agreement with the data is quite impressive. Average errors for the models are listed in Table III Surprisingly, the simple nonrelativistic BGS model is most accurate in describing the spectrum. It is somewhat disappointing that lattice has not caught up to models yet, but this may change soon.

Spectra are not particularly precise determinants of model efficacy. Other observables such as strong and leptonic widths and radiative transition rates provide sensitive probes to dynamics and it is important to consider the full set of model predictions when making comparisons.

An example of these considerations concerns the strong and leptonic widths of the $\psi(3770)$. The $\psi(3770)$ is generally assumed to be the ${ }^{3} \mathrm{D}_{1} c \bar{c}$ state; however, mixing with the $2^{3} \mathrm{~S}_{1}$ Fock component is possible and would modify the 
TABLE II: Experimental and theoretical spectrum of $c \bar{c}$ states. States assigned in this review are denoted ${ }^{a}$.

\begin{tabular}{|c|c|c|c|c|c|c|c|}
\hline State & PDG[17] & BGS[13] & GI[13] & EFG[14] & Cornell[3] & CP-PACS[15] & Chen[16] \\
\hline$\overline{J J / \psi\left(1^{3} \mathrm{~S}_{1}\right)}$ & $3096.87 \pm 0.04$ & 3090 & 3098 & 3096 & 3095 [3095] & $\overline{3085 \pm 1}$ & $3084 \pm 4$ \\
\hline$\eta_{c}\left(1^{1} \mathrm{~S}_{0}\right)$ & $2979.2 \pm 1.3$ & 2982 & 2975 & 2979 & 3095 & $3013 \pm 1$ & $3014 \pm 4$ \\
\hline$\psi^{\prime}\left(2^{3} \mathrm{~S}_{1}\right)$ & $3685.96 \pm 0.09$ & 3672 & 3676 & 3686 & 3684 [3684] & $3777 \pm 40$ & $3780 \pm 43$ \\
\hline$\eta_{c}^{\prime}\left(2^{1} \mathrm{~S}_{0}\right)$ & $3637.7 \pm 4.4$ & 3630 & 3623 & 3588 & 3684 & $3739 \pm 46$ & $3707 \pm 20$ \\
\hline$\psi\left(3^{3} \mathrm{~S}_{1}\right)$ & $4040 \pm 10$ & 4072 & 4100 & 4088 & $4110[4225]$ & - & - \\
\hline$\eta_{c}\left(3^{1} \mathrm{~S}_{0}\right)$ & & 4043 & 4064 & 3991 & 4110 & - & - \\
\hline$\psi\left(4^{3} \mathrm{~S}_{1}\right)$ & $4415 \pm 6$ & 4406 & 4450 & - & $4460[4625]$ & - & - \\
\hline$\eta_{c}\left(4^{1} \mathrm{~S}_{0}\right)$ & & 4384 & 4425 & - & 4460 & - & - \\
\hline$\chi_{2}\left(1^{3} \mathrm{P}_{2}\right)$ & $3556.18 \pm 0.13$ & 3556 & 3550 & 3556 & $3522[3523]$ & $3503 \pm 24$ & $3488 \pm 11$ \\
\hline$\chi_{1}\left(1^{3} P_{1}\right)$ & $3510.51 \pm 0.12$ & 3505 & 3510 & 3510 & $3522[3517]$ & $3472 \pm 9$ & $3462 \pm 15$ \\
\hline$\chi_{0}\left(1^{3} \mathrm{P}_{0}\right)$ & $3415.3 \pm 0.4$ & 3424 & 3445 & 3424 & 3522 & 3408 & $3413 \pm 10$ \\
\hline$h_{c}\left(1^{1} \mathrm{P}_{1}\right)$ & $3524 \pm 1^{a}$ & 3516 & 3517 & 3526 & 3522 [3519] & $3474 \pm 40$ & $3474 \pm 20$ \\
\hline$\chi_{2}\left(2^{3} \mathrm{P}_{2}\right)$ & $3931 \pm 5^{a}$ & 3972 & 3979 & 3972 & - & $4030 \pm 180$ & - \\
\hline$\chi_{1}\left(2^{3} \mathrm{P}_{1}\right)$ & & 3925 & 3953 & 3929 & - & $4067 \pm 105$ & $4010 \pm 70$ \\
\hline$\chi_{0}\left(2^{3} \mathrm{P}_{0}\right)$ & & 3852 & 3916 & 3854 & - & $4008 \pm 122$ & $4080 \pm 75$ \\
\hline$h_{c}\left(2^{1} \mathrm{P}_{1}\right)$ & & 3934 & 3956 & 3945 & - & $4053 \pm 95$ & $3886 \pm 92$ \\
\hline$\chi_{2}\left(3^{3} \mathrm{P}_{2}\right)$ & & 4317 & 4337 & - & - & - & \\
\hline$\chi_{1}\left(3^{3} \mathrm{P}_{1}\right)$ & & 4271 & 4317 & - & - & - & \\
\hline$\chi_{0}\left(3^{3} \mathrm{P}_{0}\right)$ & & 4202 & 4292 & - & - & - & \\
\hline$h_{c}\left(3^{1} \mathrm{P}_{1}\right)$ & & 4279 & 4318 & - & - & - & \\
\hline$\psi_{3}\left(1^{3} \mathrm{D}_{3}\right)$ & & 3806 & 3849 & 3815 & 3810 & - & $3822 \pm 25$ \\
\hline$\psi_{2}\left(1^{3} \mathrm{D}_{2}\right)$ & & 3800 & 3838 & 3811 & 3810 & - & $3704 \pm 33$ \\
\hline$\psi\left(1^{3} \mathrm{D}_{1}\right)$ & $3769.9 \pm 2.5$ & 3785 & 3819 & 3798 & 3810 [3755] & - & - \\
\hline$\eta_{c 2}\left(1^{1} \mathrm{D}_{2}\right)$ & & 3799 & 3837 & 3811 & 3810 & - & $3763 \pm 22$ \\
\hline$\psi_{3}\left(2^{3} \mathrm{D}_{3}\right)$ & & 4167 & 4217 & - & 4190 & - & - \\
\hline$\psi_{2}\left(2^{3} \mathrm{D}_{2}\right)$ & & 4158 & 4208 & - & 4190 & - & - \\
\hline$\psi\left(2^{3} \mathrm{D}_{1}\right)$ & $4159 \pm 20$ & 4142 & 4194 & - & 4190 [4230] & - & - \\
\hline$\eta_{c 2}\left(2^{1} \mathrm{D}_{2}\right)$ & & 4158 & 4208 & - & 4190 & - & - \\
\hline$\chi_{4}\left(1^{3} \mathrm{~F}_{4}\right)$ & & 4021 & 4095 & - & - & - & - \\
\hline$\chi_{3}\left(1^{3} \mathrm{~F}_{3}\right)$ & & 4029 & 4097 & - & - & - & $4222 \pm 140$ \\
\hline$\chi_{2}\left(1^{3} F_{2}\right)$ & & 4029 & 4092 & - & - & - & - \\
\hline$h_{c 3}\left(1^{1} \mathrm{~F}_{3}\right)$ & & 4026 & 4094 & - & - & - & $4224 \pm 74$ \\
\hline$\chi_{4}\left(2^{3} \mathrm{~F}_{4}\right)$ & & 4348 & 4425 & - & - & - & - \\
\hline$\chi_{3}\left(2^{3} \mathrm{~F}_{3}\right)$ & & 4352 & 4426 & - & - & - & - \\
\hline$\chi_{2}\left(2^{3} \mathrm{~F}_{2}\right)$ & & 4351 & 4422 & - & - & - & - \\
\hline$h_{c 3}\left(2^{1} \mathrm{~F}_{3}\right)$ & & 4350 & 4424 & - & - & - & - \\
\hline$\psi_{5}\left(1^{3} \mathrm{G}_{5}\right)$ & & 4214 & 4312 & - & - & - & - \\
\hline$\psi_{4}\left(1^{3} \mathrm{G}_{4}\right)$ & & 4228 & 4320 & - & - & - & - \\
\hline$\psi_{3}\left(1^{3} \mathrm{G}_{3}\right)$ & & 4237 & 4323 & - & - & - & - \\
\hline$\eta_{c 4}\left(1^{1} \mathrm{G}_{4}\right)$ & & 4225 & 4317 & - & - & - & - \\
\hline
\end{tabular}

TABLE III: Average Model Errors.

\begin{tabular}{cccccc}
\hline BGS & GI & EFG & Cornell & CP-PACS & Chen \\
\hline \hline $0.3 \%$ & $0.6 \%$ & $0.4 \%$ & $1.4 \%$ & $0.9 \%$ & $1.3 \%$ \\
\hline \hline
\end{tabular}

predicted strong and leptonic widths. Allowing this mixing brings a strong decay calculation into agreement with the measured width of the $\psi(3770)$ for a mixing angle of approximately $-17^{\circ}$. Assuming that leptonic widths are dominated by S-wave wavefunction components then yields the prediction[13]

$$
\left.\frac{\Gamma_{e^{+} e^{-}}(\psi(3770))}{\Gamma_{e^{+} e^{-}}(\psi(3686))}\right|_{t h y .}=0.10 \pm 0.03,
$$

which is in good agreement with the experimental value of $0.12 \pm 0.02$. 
Strong decays of mesons are driven by nonperturbative gluodynamics and are therefore (i) difficult to compute (ii) good probes of strong QCD. Unfortunately, lattice computations of strong decays are very difficult (preliminary attempts are in Ref. [20]) and one is forced to rely on models. The most popular are the '3 $P_{0}$ ' model[21] with a two-body transition operator given by

$$
\hat{H}=\left.2 m \gamma \int d^{3} x \bar{\psi} \psi\right|_{\text {nonrel }}
$$

and the Cornell model which takes Eq. 20 seriously. The Cornell model has the advantage of unifying the description of the spectrum and decays and completely specifies the strength of the decay.

Although, extensive comparisons of models have not been made, Ref. [22] finds that ${ }^{3} P_{0}$ amplitudes tend to be much larger than those due to perturbative gluon pair production, while Ref. [23] finds that the ${ }^{3} P_{0}$ model gives better predictions for amplitude ratios than does a model which creates quark pairs with ${ }^{3} S_{1}$ quantum numbers. Table IV is an attempt to partially rectify this situation by presenting the predictions of the ${ }^{3} P_{0}$ model, the Cornell model, and computations of strong widths made in a coupled channel approach to the Cornell model 3, 24]. The first two computations are made in first order perturbation theory, while the latter is nonperturbative. I shall refer to perturbative Cornell model as the $\rho K \rho$ model to highlight this difference.

The ${ }^{3} P_{0}$ and $\rho K \rho$ calculations assume SHO wavefunctions with a universal scale parameter set to $\beta=0.5 \mathrm{GeV}$; meson masses are taken as shown. The ${ }^{3} P_{0}$ model results are those of Ref. [13] with the exception that the decay strength has been taken to be $\gamma=0.35$, giving somewhat better agreement with PDG widths. The $\rho K \rho$ computation sets $K(r)=-3 / 4 b r$ and uses a string tension of $b=0.18 \mathrm{GeV}^{2}$. The last column displays an attempt to extract widths from the coupled channel Cornell model (state masses and wavefunctions only approximate those used in the other models). Table IV also lists PDG measured widths and the results of a recent reanalysis of $R$ by Seth 25$]$.

The $\rho K \rho$ and Cornell computations should agree in the event that similar wavefunctions and meson masses were employed and the resulting width is not too large. The few cases where it is possible to make a comparison indicate rough agreement between the computations, although more extensive calculations should be made. It appears that the predicted $\rho K \rho$ widths are always smaller (ranging from a factor of 2 to a factor of 10 ) than those of the ${ }^{3} P_{0}$ model. Finally comparing ${ }^{3} P_{0}$ and $\rho K \rho$ to (the rather sparse) data indicates that the ${ }^{3} P_{0}$ model tends to over-predict decay rates while the $\rho K \rho$ model under-predicts them. It is clear that more theoretical and experimental effort is required before this situation can be clarified.

Finally, we compare the ${ }^{3} P_{0}$ model to all well measured rates in the PDG in Fig. $2{ }^{3}$. As can be seen, the computation of strong widths is not as reliable as the computation of masses; a typical width error is $30 \%$ and can reach factors of 2 or even 3 .

\footnotetext{
3 The decay modes are as follows. [1] $b_{1} \rightarrow \omega \pi,[2] \pi_{2} \rightarrow f_{2} \pi,[3] K_{0} \rightarrow K \pi,[4] \rho \rightarrow \pi \pi,[5] \phi \rightarrow K \bar{K},[6] \pi_{2} \rightarrow \rho \pi,[7] \pi_{2} \rightarrow K^{*} \bar{K}+c c$, [8] $\pi_{2} \rightarrow \omega \rho,[9] \phi(1680) \rightarrow K^{*} \bar{K}+c c,[10] K^{*} \rightarrow K \pi,[11] K^{* \prime} \rightarrow K \pi$, [12] $K^{* \prime} \rightarrow \rho K$, [13] $K^{* \prime} \rightarrow K^{*} \pi,[14] D^{*+} \rightarrow D^{0} \pi^{+}$, [15] $\psi(3770) \rightarrow D \bar{D},[16] f_{2} \rightarrow \pi \pi,[17] f_{2} \rightarrow K \bar{K},[18] a_{2} \rightarrow \rho \pi,[19] a_{2} \rightarrow \eta \pi,[20] a_{2} \rightarrow K \bar{K},[21] f_{2}^{\prime} \rightarrow K \bar{K},[22] D_{s 2} \rightarrow D K+D^{*} K+D_{s} \eta$, [23] $K_{2} \rightarrow K \pi,[24] K_{2} \rightarrow K^{*} \pi,[25] K_{2} \rightarrow \rho K,[26] K_{2} \rightarrow \omega K,[27] \rho_{3} \rightarrow \pi \pi,[28] \rho_{3} \rightarrow \omega \pi,[29] \rho_{3} \rightarrow K \bar{K},[30] K_{3} \rightarrow \rho K,[31]$ $K_{3} \rightarrow K^{*} \pi,[32] K_{3} \rightarrow K \pi$.
} 
TABLE IV: Open-flavor Strong Decays of Selected Charmonia. Widths assigned in this review are denoted ${ }^{a}$.

\begin{tabular}{|c|c|c|c|c|c|c|}
\hline Meson & Mode & PDG & Seth & ${ }^{3} P_{0}$ & $\rho K \rho$ & Cornell \\
\hline \multirow[t]{3}{*}{$\overline{\chi_{2}^{\prime}(3972)\left[2^{3} P_{2}\right]}$} & $\overline{D D \bar{D}}$ & & & 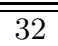 & $\overline{\overline{8.4}}$ & $\overline{\approx \approx 9}$ \\
\hline & $D D^{*}$ & & & 28 & 6.5 & $\approx 5$ \\
\hline & $D_{s} D_{s}$ & & & 0.5 & 0.1 & - \\
\hline \multirow{4}{*}{$\overline{\chi_{2}^{\prime}(3930)\left[2^{3} P_{2}\right]}$} & $\overline{D D}$ & & & 26 & & \\
\hline & $D D^{*}$ & & & 9 & & \\
\hline & $D_{s} D_{s}$ & & & - & & \\
\hline & total & $20 \pm 10^{a}$ & & 35 & & \\
\hline$\chi_{1}^{\prime}(3925)\left[2^{3} P_{1}\right]$ & $D \bar{D}^{*}$ & & & 127 & 16 & $\approx 130$ \\
\hline$\chi_{0}^{\prime}(3852)\left[2^{3} P_{0}\right]$ & $D D$ & & & 23 & 4 & $\approx 60$ \\
\hline$h_{c}(3934)\left[2^{1} P_{1}\right]$ & $D \bar{D}^{*}$ & & & 67 & 9 & $\approx 65$ \\
\hline$\psi_{3}(3806)\left[1^{3} D_{3}\right]$ & $D D$ & & & 0.3 & 0.04 & $\approx 0.2$ \\
\hline$\psi(3770)\left[1^{3} D_{1}\right]$ & $D \bar{D}$ & $24 \pm 3$ & & 33 & 11 & 20 \\
\hline \multirow[t]{6}{*}{$\overline{\psi(4159)\left[2^{3} D_{1}\right]}$} & $D D$ & & & 12 & & \\
\hline & $D \bar{D}^{*}$ & & & 0.3 & & \\
\hline & $D^{*} \bar{D}^{*}$ & & & 27 & 2.6 & \\
\hline & $D_{s} \bar{D}_{s}$ & & & 6 & & \\
\hline & $D_{s}^{*} \bar{D}_{s}^{*}$ & & & 11 & & \\
\hline & total & $78 \pm 20$ & $107 \pm 8$ & 57 & & \\
\hline \multirow{4}{*}{$\overline{\eta_{c 2}^{\prime}(4158)\left[2^{1} D_{2}\right]}$} & $D \bar{D}^{*}$ & & & 39 & & \\
\hline & $D^{*} \bar{D}^{*}$ & & & 33 & & \\
\hline & $D_{s} \bar{D}_{s}^{*}$ & & & 14 & & \\
\hline & & & & 85 & & \\
\hline \multirow[t]{4}{*}{$\psi_{2}^{\prime}(4158)\left[2^{3} D_{2}\right]$} & $D \bar{D}^{*}$ & & & 24 & & \\
\hline & $D^{*} \bar{D}^{*}$ & & & 25 & & \\
\hline & $D_{s} \bar{D}_{s}^{*}$ & & & 20 & & \\
\hline & total & & & 69 & & \\
\hline \multirow[t]{6}{*}{$\overline{\psi_{3}^{\prime}(4167)\left[2^{3} D_{3}\right]}$} & $D \bar{D}$ & & & 18 & & \\
\hline & $D \bar{D}^{*}$ & & & 39 & & \\
\hline & $D^{*} \bar{D}^{*}$ & & & 52 & & \\
\hline & $D_{s} \bar{D}_{s}$ & & & 4 & & \\
\hline & $D_{s} \bar{D}_{s}^{*}$ & & & 1 & & \\
\hline & total & & & 114 & & \\
\hline \multirow[t]{5}{*}{$\overline{\psi(4040)\left[3^{3} S_{1}\right]}$} & $D D$ & & & 0.1 & 2.3 & \\
\hline & $D \bar{D}^{*}$ & & & 25 & 17 & \\
\hline & $D^{*} \bar{D}^{*}$ & & & 25 & 5 & \\
\hline & $D_{s} \bar{D}_{s}$ & & & 6 & 1.6 & \\
\hline & total & $52 \pm 10$ & $88 \pm 5$ & 57 & 26 & \\
\hline \multirow[t]{3}{*}{$\overline{\eta_{c}^{\prime \prime}(4043)\left[3^{1} S_{0}\right]}$} & $D \bar{D}^{*}$ & & & 36 & 25 & \\
\hline & $D^{*} \bar{D}^{*}$ & & & 25 & 6 & \\
\hline & total & & & 61 & 31 & \\
\hline \multirow[t]{5}{*}{$\overline{\psi(4415)\left[4^{3} S_{1}\right]}$} & $D^{*} \bar{D}^{*}$ & & & 12 & & \\
\hline & $D \bar{D}_{1}$ & & & 24 & & \\
\hline & $D \bar{D}_{2}$ & & & 18 & & \\
\hline & $D_{s} \bar{D}_{s}^{*}$ & & & 2 & & \\
\hline & total & $43 \pm 15$ & $119 \pm 15$ & 60 & & \\
\hline
\end{tabular}




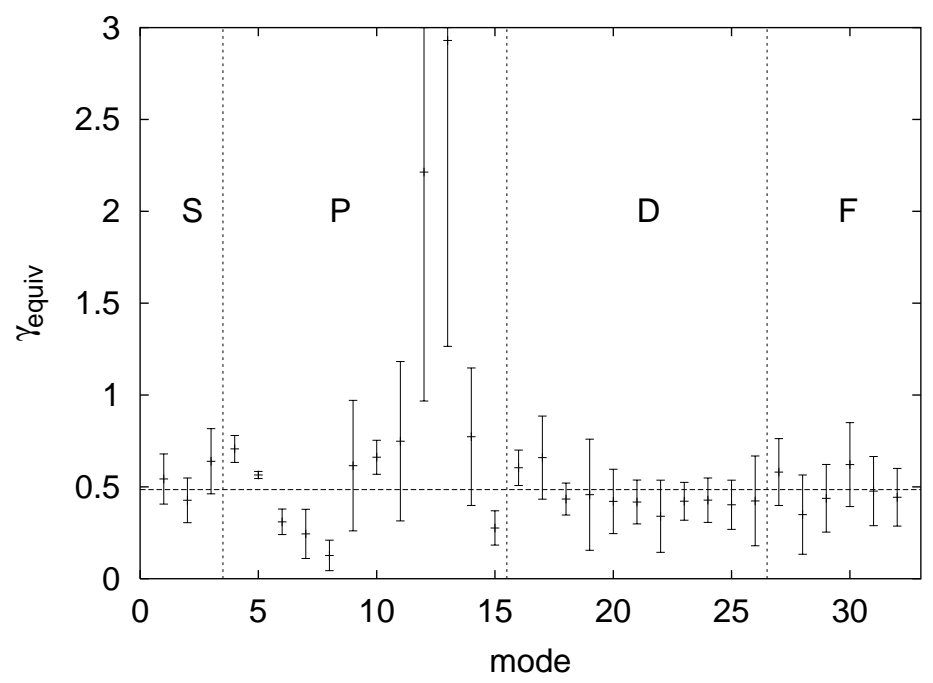

FIG. 2: ${ }^{3} P_{0}$ Couplings Required to Reproduce Data 26].

\section{Critique}

\section{Constituent Quark Models}

Most complaints about constituent quark models centre on one theme: quark models are not QCD. But models do not seek to be QCD, rather they attempt to capture the dominant physics relevant to the problem at hand; in this case, the structure and dynamics of hadrons. In this regard, most quark models suffer from serious deficiencies which will be become apparent as states higher in the spectrum are discovered. Namely: (i) Fock sector mixing (or coupled channel effects) will become more important as more continuum thresholds open up. Of course virtual meson loops also impact the low lying spectrum, but these effects are more easily absorbed into model parameters 27. (ii) Although gluons may be integrated out to good accuracy low in the spectrum, they must manifest themselves as dynamical degrees of freedom somewhere (roughly $1300 \mathrm{MeV}$ above the lowest S-wave states in a given flavour sector). (iii) The use of chirally non-invariant effective interactions (for example, scalar confinement and the ${ }^{3} P_{0}$ model) cannot be accurate for systems with typical momentum scales well above the chiral symmetry breaking and quark mass scales 28 .

\section{Short Range Interactions}

Although one may hope that short range quark interactions are described by one gluon exchange, this is far from obvious in the intermediate regime relevant to hadrodynamics. In particular, spin-dependence in the quark interaction may arise due to one gluon exchange, coupled channel effects, relativistic dynamics, and instantons. The last three remain serious challenges for model builders.

A good example of the difficulties which remain to be surmounted is the use of the scalar confinement interaction discussed in Section[B 1. Among its many problems are that it breaks chiral symmetry (and therefore is only useful as an effective interaction low in the spectrum where explicit chiral symmetry breaking is relevant) and that it cannot be used to describe baryons (quark-quark scalar interactions are of opposite sign to quark-antiquark scalar interactions, implying that baryons are dominated by an unphysical negative long range interaction). Indeed the entire analysis of Section [B 1 was much too naive: QCD does not generate quark interactions of the structure $\int J K J$. In fact, spin-dependent interactions arise from nonperturbative gluodynamics involving virtual hybrid states. This can lead to complex behaviour - including permitting a vector confinement potential to have an effective scalar spin-dependent interaction. How this can be achieved is illustrated in Ref. [29].

\section{Lattice}

Although lattice gauge theory is 'in principle' equivalent to QCD, in practice it has far to go. A simple way to see this is by counting parameters - 'in principle' there should only be one parameter necessary for all lattice computations, $\alpha_{s}$, or equivalently, $\Lambda_{Q C D}$. In practice there are many hidden parameters, including the lattice spacing, the lattice volume, the structure of the interpolating field (which typically must be carefully tuned to obtain a signal), Monte Carlo parameters (number of sweeps, Metropolis parameters, etc), autocorrelation parameters, chiral tuning 
parameters, plateau parameters, etc. Of course the benefit of the lattice is that all of these dependences may be categorised as systematic errors and eventually reduced; something which cannot be said of models.

However, more serious concerns remain. The computations reported in Table 1 employed the quenched approximation, which unfortunately is not an approximation, but a truncation which renders QCD a sick theory. Furthermore, disconnected diagrams are rarely included in spectrum computations. Although we look forward to the day when these issues are overcome a warning is appropriate: unquenched lattice computations permit hadron decay, both virtual and real. Accommodating these in a rigorous way can only be achieved by computing observables such as scattering matrix elements. This represents a fearsome technical challenge. It also implies that full lattice computations will require all of the techniques of (and be subject to all of the ambiguities associated with) experimental partial wave analysis. Finally, it is worth stating that quenched lattice computations tend to succeed where constituent quark models succeed and fail where they fail. The convergence is not surprising: quark models fail when states have a complicated Fock structure and the lattice has difficulty with such states because they represent multiple scale unquenched systems.

\section{Decay Models}

The importance of continuum channels on the spectrum and hadronic properties has already been mentioned. Of course attempts to describe such physics relies on a thorough understanding of the transition operator which mixes Fock sectors. As discussed above, our understanding of these operators is essentially nonexistent. This leads to a variety of issues when attempting to 'unquench' the quark model.

First, as opposed to quark model lore, continuum thresholds affect states throughout the spectrum. However, these effects may be largely renormalised away leaving residual effects near thresholds. Of course, the problem in hadronic physics is that continuum channels tend to get dense above threshold. Summing over these channels is nontrivial ${ }^{4}-$ indeed the sum may not converge.

Furthermore, one expects that when the continuum virtuality is much greater than $\Lambda_{Q C D}$ quark-hadron duality will be applicable and the sum over hadronic channels should evolve into perturbative quark loop corrections to the Wilson loop or quark model potential. Correctly incorporating this into constituent quark models requires marrying QCD renormalisation with effective models and is not a simple task.

Finally, pion and multipion loops can be expected to dominate the virtual continuum component of hadronic states (where allowed) due to the light pion mass. This raises the issue of correctly incorporating chiral dynamics into unquenched quark models. The relationship of chiral symmetry breaking to the constituent quark model has been discussed in Ref. [30] and a variety of hadronic models which incorporate chiral symmetry breaking exist 31] but much remains to be achieved.

\footnotetext{
${ }^{4}$ Note that the Cornell group only sum over six continuum channels, $D \bar{D}, D \bar{D}^{*}, D^{*} \bar{D}^{*}, D_{s} \bar{D}_{s}, D_{s} \bar{D}_{s}^{*}$, $D_{s}^{*} \bar{D}_{s}^{*}$; the sensitivity of their results to this truncation should be tested.
} 


\section{THE $\mathrm{X}(3872)$}

The $X(3872)$ is the poster boy of the new heavy hadrons - it has been observed by four experiments in three decay and two production channels and continues to refuse to fit into our expectations for charmonium. Experimental properties of the $X$ will be reviewed in the next subsection. Subsequent subsections will present model and effective field theory attempts at understanding the $X$. Finally, other heavy quark molecular states are considered.

\section{A. Experiment}

A summary of the discovery modes of the $X(3872)$ is presented in Table $\nabla$

TABLE V: $X(3872)$ Discovery Modes.

\begin{tabular}{cclccc}
\hline mass & width & production/decay mode & events & significance experiment \\
\hline \hline $3872.0 \pm 0.6 \pm 0.5$ & $<2.390 \%$ C.L. & $B^{ \pm} \rightarrow K^{ \pm} X \rightarrow K^{ \pm} \pi^{+} \pi^{-} J / \psi$ & $25.6 \pm 6.8$ & $10 \sigma$ & Belle[32] \\
$3871.3 \pm 0.7 \pm 0.4$ & resolution & $p \bar{p} \rightarrow X \rightarrow \pi^{+} \pi^{-} J / \psi$ & $730 \pm 90$ & $11.6 \sigma$ & CDFII[33] \\
$M(J / \psi)+774.9 \pm 3.1 \pm 3.0$ & resolution & $p \bar{p} \rightarrow X \rightarrow \pi^{+} \pi^{-} J / \psi$ & $522 \pm 100$ & $5.2 \sigma$ & D $\varnothing[34]$ \\
$3873.4 \pm 1.4$ & - & $B^{-} \rightarrow K^{-} X \rightarrow K^{-} \pi^{+} \pi^{-} J / \psi 25.4 \pm 8.7$ & $3.5 \sigma$ & BaBar[35] \\
\hline \hline
\end{tabular}

The world average $X$ mass is

$$
M(X)=3871.9 \pm 0.5 \mathrm{MeV}
$$

assuming independent systematic errors.

The clarity of the $X$ signal is exemplified in the $\pi \pi J / \psi$ invariant mass distribution as seen by CDF-II and shown in Fig. 3

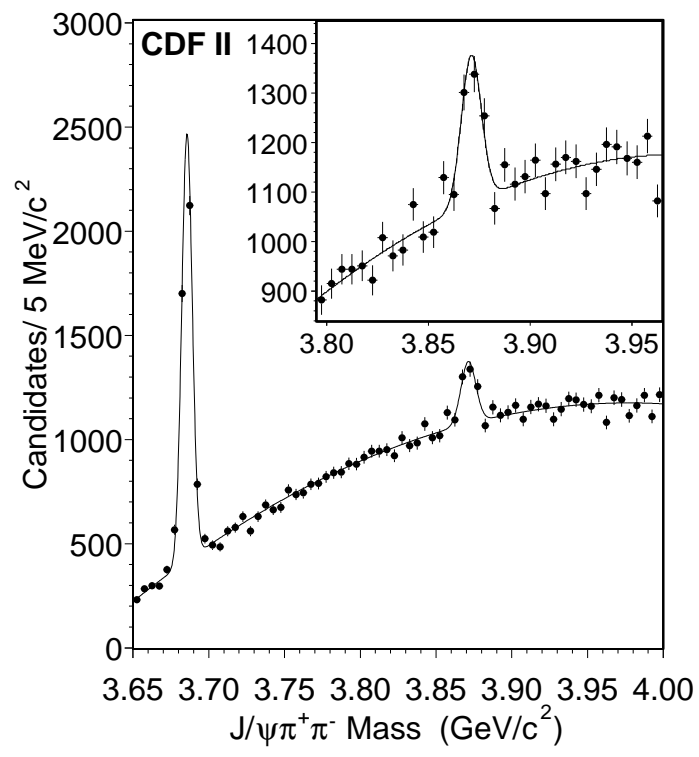

FIG. 3: The $X$ in $\pi \pi J / \psi[33]$.

As will be discussed below, the invariant mass $\pi \pi$ distribution carries important information about $X$ decays. Thus it is of interest that CDF-II have collected enough statistics to verify that the $\pi \pi$ invariant mass distribution in $X$ decays is dominated by an intermediate $\rho$ meson. This is illustrated in Fig. 4

BaBar 35] have set an upper limit on the $\eta J / \psi$ decay mode of the $X[37]$ :

$$
\operatorname{Br}\left(B^{ \pm} \rightarrow X K^{ \pm}\right) \operatorname{Br}(X \rightarrow \eta J / \psi)<7.7 \cdot 10^{-6} 90 \% \text { C.L. }
$$




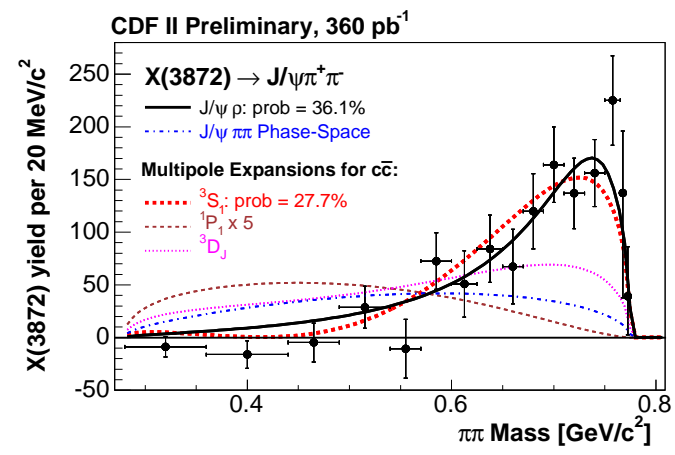

FIG. 4: $\pi \pi$ Invariant Mass Distribution [36].

The relevant data are shown in Fig. [5

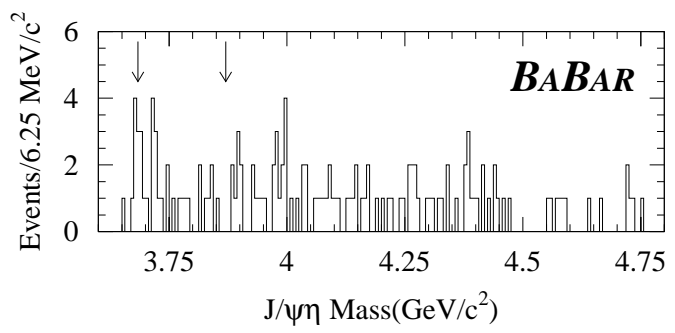

FIG. 5: Search for $X \rightarrow \eta J / \psi[37]$.

If the $X$ is an isovector state charged partners should exist and one expects that the branching ratio for the charged $X$ should be twice that of the neutral $X$. BaBar 38 have searched for these and find the limits

$$
\operatorname{Br}\left(B^{0} \rightarrow K^{+} X^{-}\right) \operatorname{Br}\left(X^{-} \rightarrow \pi^{-} \pi^{0} J / \psi\right)<5.4 \cdot 10^{-6}
$$

and

$$
\operatorname{Br}\left(B^{-} \rightarrow \bar{K}^{0} X^{-}\right) \operatorname{Br}\left(X^{-} \rightarrow \pi^{-} \pi^{0} J / \psi\right)<22 \cdot 10^{-6} .
$$

Spurred on by tetraquark models of the $X$ (see Section [1]), BaBar 39] have examined the mass difference of the $X$ s produced in charged and neutral $B$ decays and determine this to be

$$
\Delta M(X)=2.7 \pm 1.3 \pm 0.2 \mathrm{MeV} .
$$

They have also determined that the ratio of neutral to charged production of the $X$ is constrained to lie between $15 \%$ and $134 \%$ :

$$
0.15<\frac{B r\left(B^{0} \rightarrow K^{0} \pi \pi J / \psi\right)}{B r\left(B^{+} \rightarrow K^{+} \pi \pi J / \psi\right)}<1.34
$$

at $90 \%$ C.L. This result has recently been improved by BaBar [39], who measure ${ }^{5}$

$$
\frac{B r\left(B^{0} \rightarrow X K^{0}\right)}{B r\left(B^{+} \rightarrow X K^{+}\right)}=0.50 \pm 0.30 \pm 0.05
$$

\footnotetext{
${ }^{5}$ This result has been updated from the previous $0.61 \pm 0.36 \pm 0.06[40]$.
} 
Motivated by the predictions of Ref. [42], Belle have searched for and found the $X$ in the channel $\gamma J / \psi[43$ ] thus establishing that the $X$ has positive charge conjugation parity. The signal is shown in Fig. 6 and has a significance of $4 \sigma$.

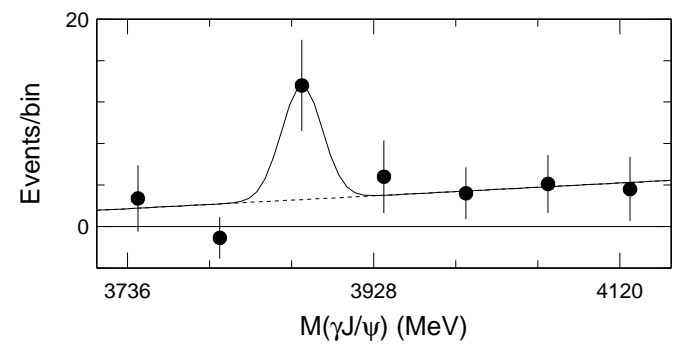

FIG. 6: Observation of $X \rightarrow \gamma J / \psi[43]$.

Belle also have measured the product of branching fractions

$$
\operatorname{Br}(B \rightarrow K X) \operatorname{Br}(X \rightarrow \gamma J / \psi)=(1.8 \pm 0.6 \pm 0.1) \cdot 10^{-6}
$$

and the ratio of widths

$$
\frac{\Gamma(X \rightarrow \gamma J / \psi)}{\Gamma\left(X \rightarrow \pi^{+} \pi^{-} J / \psi\right)}=0.14 \pm 0.05
$$

The same paper reports the discovery of the $X$ decaying to $\pi^{+} \pi^{-} \pi^{0} J / \psi$ with a significance of $4 \sigma$ (see Fig. (7) with a strength that is comparable to that of the $\pi \pi J / \psi$ mode:

$$
\frac{B r\left(X \rightarrow \pi^{+} \pi^{-} \pi^{0} J / \psi\right)}{B r\left(X \rightarrow \pi^{+} \pi^{-} J / \psi\right)}=1.0 \pm 0.4 \pm 0.3 .
$$

This observation establishes strong isospin violation effects, which will be discussed further in Section ЩB

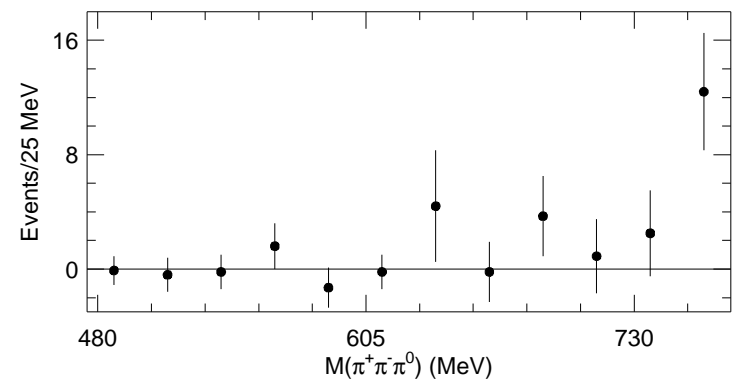

FIG. 7: Observation of $X \rightarrow \pi \pi \pi J / \psi[43]$.

The products of branching ratios have been measured by Belle $[44]$ and BaBar [39, 40]:

$$
\operatorname{Br}\left(B^{+} \rightarrow X K^{+}\right) \operatorname{Br}(X \rightarrow \pi \pi J / \psi)=(1.3 \pm 0.3) \cdot 10^{-5}
$$

and

$$
\operatorname{Br}\left(B^{+} \rightarrow X K^{+}\right) \operatorname{Br}(X \rightarrow \pi \pi J / \psi)=(1.01 \pm 0.25 \pm 0.10) \cdot 10^{-5}
$$


Finally, there are rumours that Belle observes the $X$ decaying to $D^{0} \bar{D}^{0} \pi^{0}$ at a rate ten times larger than that of $\pi \pi J / \psi[45]$. The new decay mode is seen in $11.3 \pm 3.6$ events at $5.6 \sigma$ with a product of branching fractions of

$$
\operatorname{Br}(B \rightarrow K X) \operatorname{Br}(X \rightarrow D \bar{D} \pi)=(2.2 \pm 0.7 \pm 0.4) \cdot 10^{-4} .
$$

Determining the quantum numbers of the $X$ is a high priority experimental task. BES [46] have searched for the $X$ in $e^{+} e^{-}$collisions and find the bound

$$
\Gamma\left(e^{+} e^{-}\right) \operatorname{Br}(X \rightarrow \pi \pi J / \psi)<10 \mathrm{eV}
$$

at $90 \%$ C.L. Thus the $X$ is unlikely to be a vector charmonium state. This conclusion is confirmed by Babar 41] who search for the $X$ in initial state radiation and find an upper limit to the product of branching ratios:

$$
\Gamma\left(X \rightarrow e^{+} e^{-}\right) \operatorname{Br}(X \rightarrow \pi \pi J / \psi)<6.2 \mathrm{eV}
$$

at $90 \%$ C.L.

Belle have measured angular correlations in the $\pi \pi J / \psi$ system and use these to rule out possible $J^{P C}=0^{++}$or $0^{-+}$values for the $X$. Furthermore the dipion mass distribution strongly disfavours $1^{-+}$or $2^{-+}$assignments for the $X[43]$. Thus, it is likely that the $X$ is a $1^{++}$charmonium state 44].

$\mathrm{CDF}$ 47] and $\mathrm{D} \varnothing[34]$ have examined the production characteristics of the $X$. In particular, it is found that approximately $16 \%$ of the $X$ sample at CDF comes from $B$ decay; the remainder comes from direct production or decay of short-lived particles. The mix of production sources is similar to that observed for the $\psi(2 S)$ charmonium state [4] (see Fig. 8). Thus it appears that there is no penalty for producing the $X$ in $p \bar{p}$ collisions.

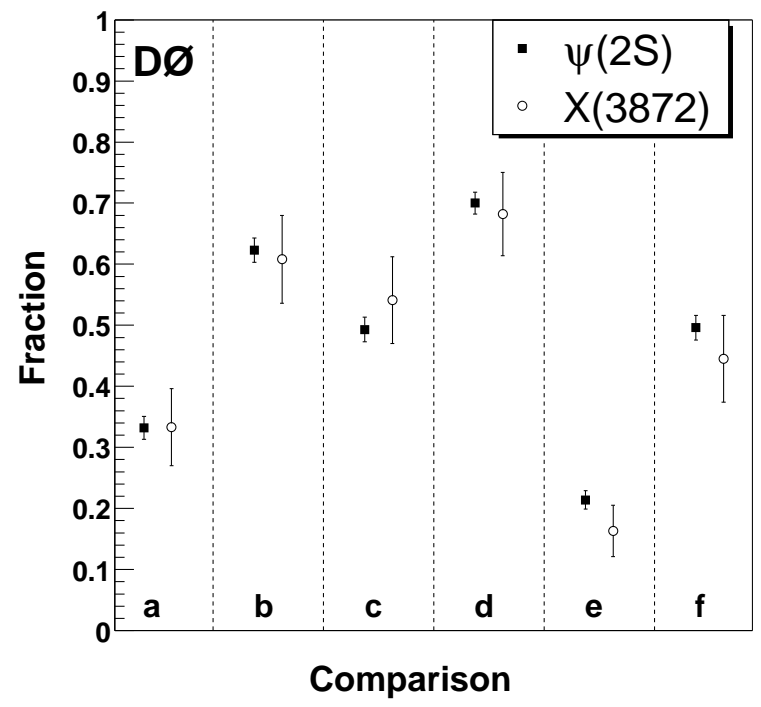

FIG. 8: Comparison of event yield fractions for $X(3872)$ and $\psi(2 S)$. (a) $p_{T}(J / \psi)>15 \mathrm{GeV},(\mathrm{b})|y|(J / \psi)<1,(\mathrm{c}) \cos \theta_{\pi}<0.4$, (d) effective proper decay length $<0.01 \mathrm{~cm}$, (e) isolation-1, (f) $\cos \theta_{\mu}<0.4$ [34].

In summary, a narrow state at $3871.9 \mathrm{MeV}$ is seen in $B$ decays and $p \bar{p}$ collisions decaying to $\pi \pi J / \psi, \pi \pi \pi J / \psi$, $\gamma J / \psi$, and $D^{0} \bar{D}^{0} \pi^{0}$ which has production characteristics similar to the $\psi(2 S)$. The quantum numbers $J^{P C}=1^{++}$ are strongly preferred by the data. Various attempts to interpret this data are presented in the following sections.

\section{B. Models}

\section{Charmonium}

The first place to seek an explanation of the $X(3872)$ is evidently in the charmonium spectrum[48]. A glance at Table $\amalg$ reveals that the only viable candidates for the $X$ are $2 P$ or $1 D$ states, with the $\chi_{c 0}^{\prime}$ being the most likely 
candidate based solely on mass. Table IV indicates that ${ }^{3} P_{0}$ widths are too large to accommodate a narrow $2 P$ or $1 D$ charmonium (however, the $\rho K \rho$ model yields smaller widths). Subsequent experimental effort (described above) has focussed attention on $1^{++}$charmonia, of which the $\chi_{c 1}^{\prime}$ is the only possible candidate. But the predicted width of this state is $127 \mathrm{MeV}$ in the ${ }^{3} P_{0}$ model and $16 \mathrm{MeV}$ in the $\rho K \rho$ model - much too large. The apparent lack of viable charmonium candidates has led to much theoretical speculation.

Three options are available when the quark model fails us: (i) attempt to change the quark model; (ii) seek supernumerary states; (iii) claim experimental or interpretational error. The first option is extremely difficult, as the average mass errors reported in Table III illustrate. In general one must find an effect which is localised in the spectrum so as not to destroy the good agreement seen elsewhere.

In the list of usual supernumerary suspects are hybrids, glueballs, diquark clusters, and molecular states. All of these options have been suggested for the $X$. The prominent ones are discussed in the following.

\section{Tetraquarks}

Maiani et al. [49] have constructed a model of the $X(3872)$ (and several other states as we shall see) which postulates that it is dominated by a diquark-diquark structure. The results of a relativistic tetraquark model are reported in Ref. 50]. Chief assumptions in the model are that quarks group in colour triplet scalar and vector clusters and that the interactions of these are dominated by a simple spin-spin interaction 51]. Using the $X(3872)$ as input, Maiani $e t$ al. predict the spectrum of new diquark charmonia shown in Fig. 9

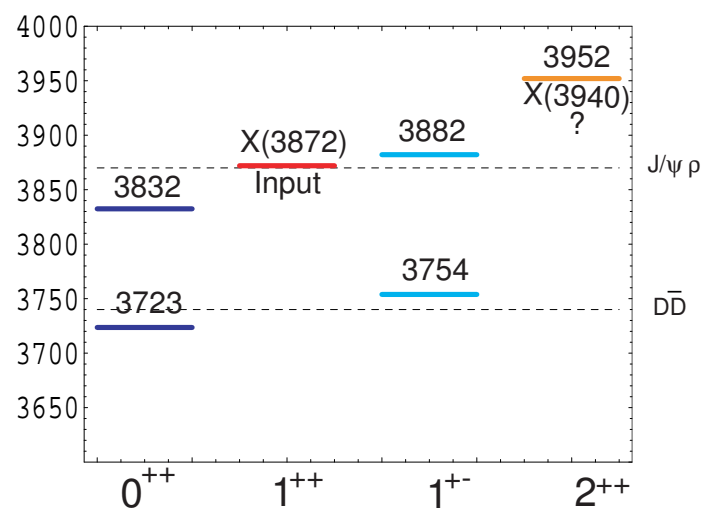

FIG. 9: Spectrum of Tetraquark Charmonium States [49].

This spectrum assumes that the mass of the scalar and vector $[c q]$ diquarks are $1933 \mathrm{MeV}$ and constructs states as follows:

$$
\begin{aligned}
\left|0^{++}\right\rangle & =\left|[c q]_{S}[\bar{c} \bar{q}]_{S} ; J=0\right\rangle \\
\left|0^{++^{\prime}}\right\rangle & =\left|[c q]_{V}[\bar{c} \bar{q}]_{V} ; J=0\right\rangle \\
\left|1^{++}\right\rangle & =\frac{1}{\sqrt{2}}\left(\left|[c q]_{S}[\bar{c} \bar{q}]_{V} ; J=1\right\rangle+\left|[c q]_{V}[\bar{c} \bar{q}]_{S} ; J=1\right\rangle\right) \\
\left|1^{+-}\right\rangle & =\frac{1}{\sqrt{2}}\left(\left|[c q]_{S}[\bar{c} \bar{q}]_{V} ; J=1\right\rangle-\left|[c q]_{V}[\bar{c} \bar{q}]_{S} ; J=1\right\rangle\right) \\
\left|1^{+-^{\prime}}\right\rangle & =\left|[c q]_{V}[\bar{c} \bar{q}]_{V} ; J=1\right\rangle \\
\left|2^{++}\right\rangle & =\left|[c q]_{V}[\bar{c} \bar{q}]_{V} ; J=2\right\rangle
\end{aligned}
$$

Thus the model predicts that charged partners of the $X(3872)$ should exist, namely $X^{+}=[c u][\bar{c} \bar{d}]$ and $X^{-}=[c d][\bar{c} \bar{u}]$. Furthermore, the two neutral $X$ states comprised of $[c u][\bar{c} \bar{u}]$ and $[c d][\bar{c} \bar{d}]$ should mix with angle $\theta$ and hence produce two neutral $X$ s with a mass difference of

$$
M\left(X_{h}\right)-M\left(X_{l}\right)=\frac{2\left(m_{d}-m_{u}\right)}{\cos (2 \theta)}=\frac{7 \pm 2}{\cos (2 \theta)} \mathrm{MeV} \approx(8 \pm 3) \mathrm{MeV}
$$


(similar considerations apply to all the states in Fig. 9). Furthermore, one of these states will populate $B^{+}$decay while the other will populate $B^{0}$ decay.

Relative strengths of the production of tetraquarks may be simply estimated. However, I disagree with the arguments of Ref. [4] where it is claimed that the amplitude for a strange quark to combine with the spectator quark is comparable or less than the amplitude for it to combine with a produced quark. Rather this amplitude is suppressed by a factor of $1 / N_{c}$ due to its colour structure. Thus one concludes that $B^{0} \rightarrow K^{-} X^{+}, B^{0} \rightarrow K^{0} X_{d}, B^{+} \rightarrow K^{+} X_{u}$, and $B^{+} \rightarrow K^{0} X^{+}$should all occur at the same rate.

The model makes strong predictions which may be compared with experiment. For example, the ratio of neutral to charged production of the $X$ is predicted to be unity whereas BaBar measure (Eq. 30) a ratio of $50 \%$ with large errors. Unfortunately, many of the other predictions of the model are not as successful. The predicted mass difference of the neutral $X \mathrm{~s} \mathrm{(Eq.} \mathrm{45)} \mathrm{is} 2 \sigma$ removed from the measured mass difference (Eq. 28) and no charged $X$ states, or any of the other states of Fig. 9 have been sighted. Finally, the existence of diquark correlations in hadrons implies an extensive new exotic and cryptoexotic spectroscopy for which very little evidence exists ${ }^{6}$.

While the ideas surrounding diquarks and tetraquarks are interesting and even compelling [52], one must reserve judgement on their reality until strong evidence for them comes to light.

\section{Additional Models}

Li has suggested that the $X$ is a $c \bar{c} g$ hybrid meson [53] which decays predominantly via $X \rightarrow J / \psi g g \rightarrow J / \psi \pi \pi$ and has a significant $J / \psi \sigma$ branching fraction. The existence of the opposite $G$-parity $3 \pi$ decay mode is explained as due to isospin violation. Its large magnitude (see Eq. 331) is left as a conundrum.

Current lattice [54] and flux tube model[55] expectations for the mass of $c \bar{c} g$ hybrids are in the range 4200-4400 $\mathrm{MeV}$, thus there must exist significant systematic error in these models to permit a charmonium hybrid at $3872 \mathrm{MeV}$. In the absence of countervailing data, one must therefore regard hybrid models of the $X$ as unlikely.

Seth 56] has proposed that the $X$ is a vector glueball with a small admixture of vector $c \bar{c}$. This proposal has since been eliminated by the observation of the $\omega J / \psi$ (Fig. 17) and $\gamma J / \psi$ (Fig. 6) decay modes.

On the basis of a simple chromomagnetic interaction model, Høgaasen et al. [57] postulate that the $X$ is a loose four quark agglomeration which is a mixture of $c u \bar{c} \bar{u}$ and $c d \bar{c} \bar{d}$ flavour states. Thus another neutral $X$ is expected along with two charged states $c u \bar{c} \bar{d}$ and $c d \bar{c} \bar{u}$. In addition, a broad isospin multiplet is predicted at $3742 \mathrm{MeV}$.

Kalashnikova has constructed a simple coupled channel model of the $c \bar{c}$ spectrum which incorporates a central confining potential and ${ }^{3} P_{0}$ model couplings to $D \bar{D}, D \bar{D}^{*}, D^{*} \bar{D}^{*}, D_{s} \bar{D}_{s}, D_{s} \bar{D}_{s}^{*}$, and $D_{s}^{*} \bar{D}_{s}^{*}$ continua [58]. Couplings are computed assuming SHO wavefunctions for the mesons and a simple renormalisation procedure is carried out. An interesting result of this computation is that the renormalised $\chi_{c 1}^{\prime}$ sits at roughly $4000 \mathrm{MeV}$, while a virtual bound state appears just above $D \bar{D}^{*}$ threshold. It is natural to identify this with the $X(3872)$, and in fact, such an identification will be indistinguishable from a very weakly bound $D \bar{D}^{*}$ molecule (to be discussed in the next section). Although this approach suffers from all of the problems mentioned in Section IB 4 and in some cases can ruin agreement with naive quark model results (such as vector meson leptonic widths), this interesting observation warrants further investigation.

Finally, Bugg [59] has reminded us that cusps occur in amplitudes at thresholds, and these can manifest themselves as bumps in cross sections slightly above threshold. The proximity of the $X$ to $D^{0} \bar{D}^{* 0}$ threshold implies that the cusp scenario should be treated seriously. In this regard, we note that typical enhancements due to cusps are $\mathcal{O}\left(\Lambda_{Q D C}\right)$ in width, in contrast with the very narrow $X$. Furthermore, if the $X$ is simply due to the opening of a threshold, then the reaction $B^{+} \rightarrow K^{0} D^{+} \bar{D}^{* 0}$ should exhibit a peak similar to the $X$ channel $B^{+} \rightarrow K^{+} D^{0} \bar{D}^{* 0}$. That it does not indicates that significant final state interactions are affecting $B^{+} \rightarrow K^{+} D^{0} \bar{D}^{* 0}$. And of course these interactions may be strong enough to create a hadronic resonance. Additional observations on distinguishing bound states from cusps can be found in Ref. [79].

\section{Molecular Interpretation of the $X$}

In principle nothing in QCD prevents the formation of nuclear-like bound states of mesons and speculation on the existence of such states dates back thirty years 60, 61]. The proximity of the $X$ to $D^{0} \bar{D}^{0 *}$ threshold immediately led to speculation that the $X$ is a $D \bar{D}^{*}$ resonance [62, 63, 64, 65, 66]. Indeed $D^{0} \bar{D}^{0 *}$ threshold is at

\footnotetext{
6 The fiascos associated with pentaquarks and baryonia will only be mentioned in this footnote.
} 


$$
D^{0} \bar{D}^{0 *}=3871.2 \pm 1.0 \mathrm{MeV}
$$

while the world average $X$ mass is (Eq. 24) $3872.0 \pm 1.8 \mathrm{MeV}, 0.7 \pm 1.2 \mathrm{MeV}$ higher than threshold. The positive binding energy (with large error) is disconcerting for molecular interpretations, but, is no problem for tetraquark, cusp, or virtual state interpretations. In any event, the binding energy for a putative molecule is very small and this forms the basis for an effective field theory approach to the $X$, which will be discussed in the following section.

In general, if the $X$ is dominated by a loose $D^{0} \bar{D}^{0 *}$ component then it does not have good isospin which should give rise to a distinct decay pattern. The most likely bound state is an S-wave which implies that the quantum numbers of the $X$ are $J^{P C}=1^{++}$. Mixing with nearby $\chi_{c 1}$ states should induce weak isospin violating decays in them as well. Finally, since the mesons are weakly bound they decay as if they were free, thus the $X$ should decay via the modes $X \rightarrow D^{0} \bar{D}^{0} \pi^{0}$ and $X \rightarrow D^{o} \bar{D}^{0} \gamma$ with a relative rate of $62: 38$.

Making more detailed predictions forces one to employ a specific model because lattice gauge theory is not sufficiently well developed to permit accurate examination of this state (although a recent attempt has appeared 67]; see Section IB4 for additional comments) and too many unknown couplings are present in effective field theory descriptions of the $X$.

The first step in constructing a molecular (or any) model of the $X$ is to determine the relevant degrees of freedom. The $D^{0} \bar{D}^{* 0}$ channel has already been identified as important. This in turn implies that the binding energy is very small which implies that $c \bar{c}$ components in the $X$ are small (this is quantified below). Similarly excluding hybrid components leaves meson-meson channels. Of these the four closest are listed in Table $\mathbf{D T}$

TABLE VI: Continuum Channels near $3872 \mathrm{MeV}$.

\begin{tabular}{llcc}
\hline channel & threshold & $\Gamma(A)$ & $\Gamma(B)$ \\
\hline \hline$D^{0} \bar{D}^{* 0}$ & 3871.2 & - & $<2.1 \mathrm{MeV}$ \\
$D^{+} D^{*-}$ & 3879.3 & - & $96 \pm 22 \mathrm{keV}$ \\
$\rho^{0} J / \psi$ & 3867.9 & $150.3 \pm 1.6 \mathrm{MeV}$ & $91.0 \pm 3.2 \mathrm{keV}$ \\
$\omega J / \psi$ & 3879.5 & $8.49 \pm 0.08 \mathrm{MeV}$ & $"$ \\
\hline \hline
\end{tabular}

The next element in a microscopic description is dynamics. Two candidates come to mind: pion exchange and quark-level interactions. Indeed, if quark dynamics are limited to quark/colour exchange interactions then it is natural to admit both interactions since pion exchange is long range while quark exchange is a short range effect.

The quark model employed here assumes nonrelativistic quark dynamics mediated by an instantaneous confining interaction and a short range spin-dependent interaction motivated by one gluon exchange. The colour structure is taken to be the quadratic form of perturbation theory. This is an important assumption for multiquark dynamics which has received support from recent lattice computations for both confinement [68] and multiquark interactions [69]. The final form of the interaction is thus taken to be

$$
\sum_{i<j} \frac{\boldsymbol{\lambda}(i)}{2} \cdot \frac{\boldsymbol{\lambda}(j)}{2}\left\{\frac{\alpha_{s}}{r_{i j}}-\frac{3}{4} b r_{i j}-\frac{8 \pi \alpha_{s}}{3 m_{i} m_{j}} \boldsymbol{S}_{i} \cdot \boldsymbol{S}_{j}\left(\frac{\sigma^{3}}{\pi^{3 / 2}}\right) e^{-\sigma^{2} r_{i j}^{2}}\right\}
$$

where $\boldsymbol{\lambda}$ is a colour Gell-Mann matrix, $\alpha_{s}$ is the strong coupling constant, $b$ is the string tension, $m_{i}$ and $m_{j}$ are the interacting quark or antiquark masses, and $\sigma$ is a range parameter in a regulated spin-spin hyperfine interaction. The parameters used were $\alpha_{s}=0.59, b=0.162 \mathrm{GeV}^{2}, \sigma=0.9 \mathrm{GeV}$, and $0.335,0.55$, and $1.6 \mathrm{GeV}$ for up, strange, and charm quark masses respectively. Relevant meson masses obtained from this model are $\rho=0.773 \mathrm{GeV}, J / \psi=3.076$ $\mathrm{GeV}, D=1.869 \mathrm{GeV}$, and $D^{*}=2.018 \mathrm{GeV}$, in good agreement with experiment.

Meson-meson interactions are obtained by computing the Born order scattering amplitude shown in Fig. 10] for a given process [70]. Because of the colour factors in Eq. 47 this amplitude necessarily involves an exchange of quarks between the interacting mesons. Thus the leading order $D \bar{D}^{*}$ interaction couples $D \bar{D}^{*}$ with hidden charm states such as $\rho J / \psi$ and $\omega J / \psi$. This amplitude may be unitarised by extracting an effective potential and iterating it in a Schrödinger equation 70]. The method has been successfully applied to a variety of processes such as $K N$ scattering [71] and $J / \psi$ reactions relevant to RHIC physics [72]. It has even proven surprisingly useful for relativistic (and chiral) reactions such as $\pi \pi$ scattering 70 .

An effective potential is extracted by equating the scattering amplitude to that obtained for point-like mesons interacting via an arbitrary S-wave potential. The resulting potential is shown in Fig. 11] The distinctive "mermaid potential" seen here is due to destructive interference between diagrams in the quark level amplitude. Thus details of 

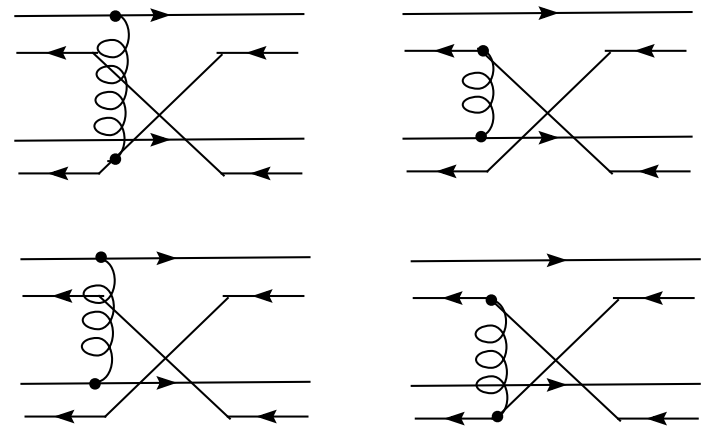

FIG. 10: Quark Exchange Diagrams Contributing to Meson-Meson Scattering.

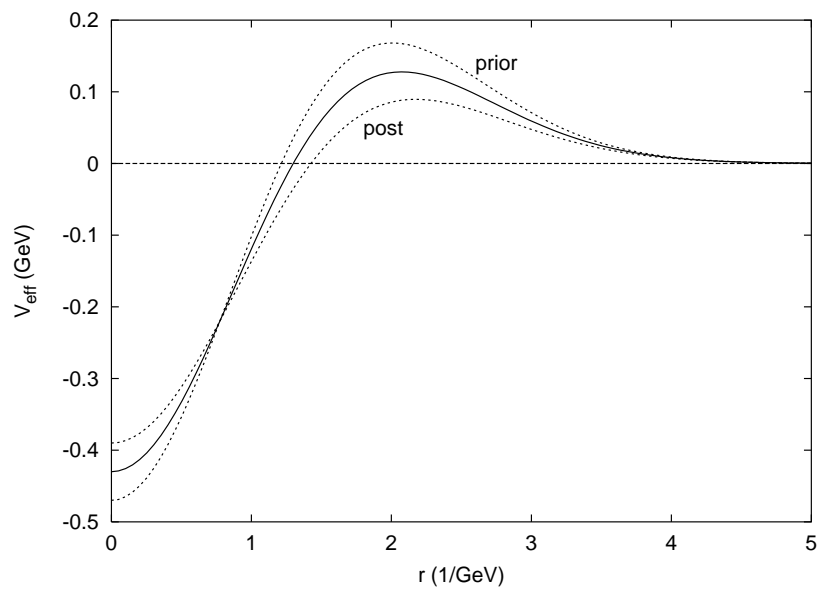

FIG. 11: Effective Potential for $D \bar{D}^{*} \rightarrow \omega J / \psi$. The dashed lines indicate different partitions of the four-quark Hamiltonian.

the potential are sensitive to the assumed microscopic interaction, however, its general shape and strength are quite robust.

Long range interactions are assumed to be dominated by one-pion-exchange. Ref. [66] chose to follow the method of Törnqvist 61] in constructing an effective pion-induced interaction. This is based on a microscopic quark-pion interaction familiar from nuclear physics (in more modern language, the form of the interaction is dictated by spontaneous chiral symmetry breaking):

$$
L=-\frac{g}{\sqrt{2} f_{\pi}} \int d^{3} x \bar{\psi}(x) \gamma^{\mu} \gamma_{5} \tau^{a} \psi(x) \partial_{\mu} \pi^{a}(x) .
$$

Here $f_{\pi}=92 \mathrm{MeV}$ is the pion decay constant, $\tau$ is an $\mathrm{SU}(2)$ flavour generator, and $g$ is a coupling to be determined. The effective potential is derived by projecting the quark level interactions onto hadronic states in the nonrelativistic limit. In the case of pseudoscalar-vector states one obtains [61] (see Appendix B for more details):

$$
V_{\pi}=-\gamma V_{0}\left[\left(\begin{array}{ll}
1 & 0 \\
0 & 1
\end{array}\right) C(r)+\left(\begin{array}{cc}
0 & -\sqrt{2} \\
-\sqrt{2} & 1
\end{array}\right) T(r)\right]
$$

where

$$
\begin{gathered}
C(r)=\frac{\mu^{2}}{m_{\pi}^{2}} \frac{\mathrm{e}^{-\mu r}}{m_{\pi} r}, \\
T(r)=C(r)\left(1+\frac{3}{\mu r}+\frac{3}{(\mu r)^{2}}\right),
\end{gathered}
$$


and

$$
V_{0} \equiv \frac{m_{\pi}^{3}}{24 \pi} \frac{g^{2}}{f_{\pi}^{2}} \approx 1.3 \mathrm{MeV}
$$

The matrix elements refer to S- and D-wave components of the pseudoscalar-vector state in analogy with the deuteron. The strength of the interaction has been fixed by comparing to the $\pi N N$ coupling constant via the relationship $f_{\pi N}^{2} / 4 \pi=25 / 18 \cdot m_{\pi}^{2} g^{2} / f_{\pi}^{2}$. This allows a prediction of the $D^{*}$ decay width which is in good agreement with experiment 61]. The parameter $\mu$ is typically the pion mass, however, one can incorporate recoil effects in the potential by setting $\mu^{2}=m_{\pi}^{2}-\left(m_{V}-m_{p S}\right)^{2}$. Finally, the coupling $\gamma$ is a spin-flavour matrix element which takes on the following values: $\gamma=3$ for $I=0, C=+; \gamma=1$ for $I=1, C=-; \gamma=-1$ for $I=1, C=+$; and $\gamma=-3$ for $I=0, C=-$. Thus the isoscalar positive charge parity channel is the most likely to form bound states and subsequent discussion focusses on it.

The potential of Eq. 51 is often regulated to represent the effect of additional unknown short distance interactions. The regulator scale, $\Lambda$ can be fixed by comparison with nuclear physics; for example $N N$ interactions yield preferred values for $\Lambda$ in the range $0.8 \mathrm{GeV}$ to $1.5 \mathrm{GeV}$ depending on model details. Alternatively, reproducing the deuteron binding energy requires $\Lambda \approx 0.8 \mathrm{GeV}$. Törnqvist has employed an intermediate value of $\Lambda=1.2 \mathrm{GeV}$ which is appropriate for $D$ mesons and this is taken as the canonical cutoff in the following.

Unfortunately, $D$ mesons are sufficiently light that the $D \bar{D}^{*}$ system (just barely) does not bind with canonical parameters. However, the combined pion and quark induced effective interactions are sufficient to cause binding. Thus it is of interest to study the properties of possible bound states as a function of their binding energy. This has been achieved by allowing the regulator scale to vary between 1.2 and $2.3 \mathrm{GeV}$. Binding is seen to occur for $\Lambda$ larger than approximately $1.23 \mathrm{GeV}$.

Wavefunction probabilities are defined by

$$
Z_{\alpha}=\int\left|\varphi_{\alpha}\right|^{2}
$$

where $\alpha$ is a channel index. These are shown as a function of binding energy in Fig. 12 It is clear that the $D^{0} \bar{D}^{0 *}$ component dominates the wavefunction, especially near threshold. However, the $D^{+} D^{-*}$ component rises rapidly in strength with isospin symmetry being recovered at surprisingly small binding energies (on the order of $30 \mathrm{MeV}$ ). Alternatively, the $\omega J / \psi$ component peaks at roughly $17 \%$ at $E_{B} \approx 9 \mathrm{MeV}$. The contribution of the $\rho J / \psi$ wavefunction remains small, peaking at less than $1 \%$ very close to threshold.

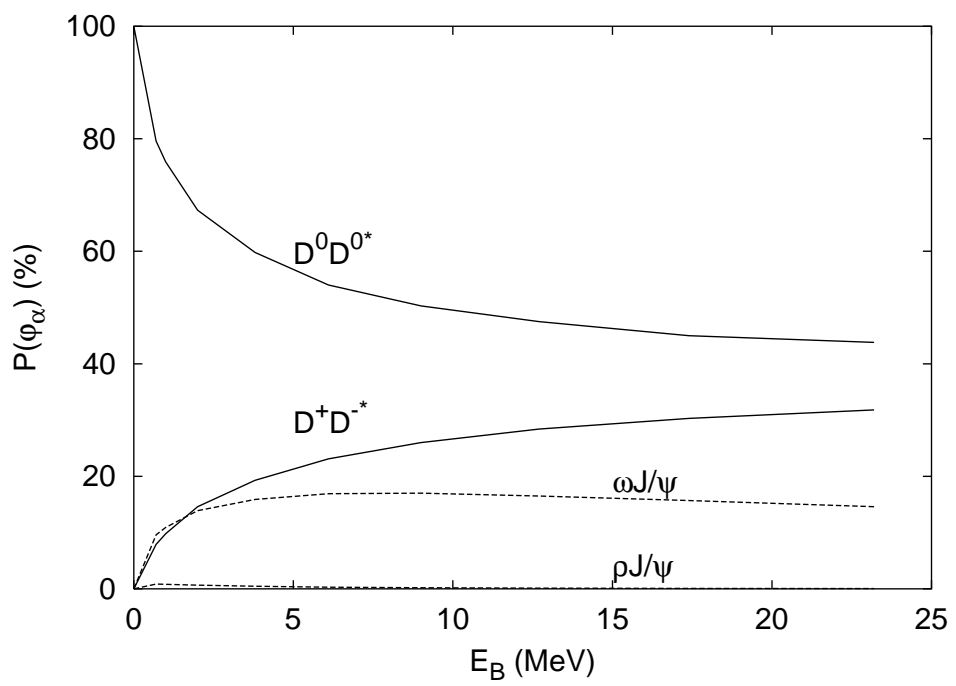

FIG. 12: Wavefunction Components of the $X[66]$.

At the time of publication this model had two post-dictions: a charmonium resonance of mass $3872 \mathrm{MeV}$ with a decay mode of $\pi \pi J / \psi$. Predictions included the quantum numbers $J^{P C}=1^{++}$; that the $\pi \pi$ invariant mass distribution should reflect its origins as a virtual $\rho$; and a $3 \pi J / \psi$ decay mode of strength comparable to the $2 \pi$ mode with an invariant mass distribution dominated by the $\omega$. All of these predictions were subsequently confirmed experimentally (see Figs 4 17 and Eq. 331. Decays are discussed in Sect. [ID Further data and discussion of the molecular interpretation of the $X$ appears in Sect. ЩE 


\section{Effective Field Theory}

The notion that the $X$ is dominated by a weakly bound $D^{0} \bar{D}^{* 0}$ system suggests that an effective field theory approach to the $X$ may be useful, in analogy to the successful description of the deuteron $[73]$. The utility of this approach is further enhanced by an approximate heavy quark symmetry and the anomalously small binding energy of $E_{B}=-0.5 \pm 0.9 \mathrm{MeV}$, which should be compared to the natural scale of $m_{\pi} /\left(2 \mu_{D D^{*}}\right) \approx 20 \mathrm{MeV}[74$, 75].

Weakly bound systems may be described in terms of a single parameter, the scattering length $a-$ a property known as 'low energy universality'. Simple results which apply to a weakly bound system include estimates of binding energy

$$
E_{B}=\frac{1}{2 \mu a^{2}}
$$

and the weak binding wavefunction

$$
\psi(r)=\frac{\mathrm{e}^{-r / a}}{\sqrt{2 \pi a} r}
$$

or

$$
\tilde{\psi}(k)=\sqrt{\frac{8 \pi}{a}} \frac{1}{k^{2}+a^{-2}} .
$$

The scattering lengths can be quite large (compare to the deuteron, $a_{D}=4.3 \mathrm{fm}$ ). For example, $E_{B}=0.5 \mathrm{MeV}$ corresponds to $a=6.4 \mathrm{fm}$, while $E_{B}=0.1 \mathrm{MeV}$ implies that $a=14.4 \mathrm{fm}^{7}$. Universality ideas were first exploited by Voloshin [4] who estimated the effects of interference on the processes $X \rightarrow D^{0} \bar{D}^{0} \gamma$ and $X \rightarrow D^{0} \bar{D}^{0} \pi^{0}$.

Braaten and Kusunoki have followed this initial work with a series of papers examining production and decay of the $X$. This began with the observation that the probabilities for non- $D^{0} \bar{D}^{* 0}$ components of the $X, X$ production, and $X$ decays all scale as $1 / a$ and hence are strongly suppressed in the weak binding limit.

The analysis was performed with the aid of an effective field theory which couples $D, D^{*}$ and $\chi$ mesons 75$]$ :

$$
\begin{aligned}
\mathcal{H}= & m_{D^{0}}\left(D^{\dagger} D+\bar{D}^{\dagger} \bar{D}\right)+m_{D^{* 0}}\left(\mathbf{D}^{\dagger} \cdot \mathbf{D}+\overline{\mathbf{D}}^{\dagger} \cdot \overline{\mathbf{D}}\right) \\
& +\left(m_{D^{0}}+m_{D^{* 0}}+\nu_{0}\right) \chi^{\dagger} \cdot \boldsymbol{\chi} \\
- & \frac{1}{2 m_{D^{0}}}\left(D^{\dagger} \nabla^{2} D+\bar{D}^{\dagger} \nabla^{2} \bar{D}\right)-\frac{1}{2 m_{D^{* 0}}}\left(\mathbf{D}^{\dagger} \cdot \nabla^{2} \mathbf{D}+\overline{\mathbf{D}}^{\dagger} \cdot \nabla^{2} \overline{\mathbf{D}}\right) \\
& -\frac{1}{2}\left(m_{D^{0}}+m_{D^{* 0}}\right)^{-1} \boldsymbol{\chi}^{\dagger} \cdot \nabla^{2} \boldsymbol{\chi} \\
+ & \lambda_{0}(D \overline{\mathbf{D}}+\bar{D} \mathbf{D})^{\dagger} \cdot(D \overline{\mathbf{D}}+\bar{D} \mathbf{D}) \\
& +g_{0}\left[\boldsymbol{\chi}^{\dagger} \cdot(D \overline{\mathbf{D}}+\bar{D} \mathbf{D})+(D \overline{\mathbf{D}}+\bar{D} \mathbf{D})^{\dagger} \cdot \boldsymbol{\chi}\right]
\end{aligned}
$$

The bare $\chi$ binding energy is represented by $\nu_{0}$. The four body interaction of strength $\lambda_{0}$ represents short range interactions between $D$ and $D^{*}$ mesons while the term proportional to $g_{0}$ couples the $\chi$ to its constituent $D \bar{D}^{*}$ channel via a pointlike interaction.

Low energy universality also permits analysis of 'coalescence' models of $X$ production in $\Upsilon$ and $B$ decays (see Fig. 13) with the results 76

$$
\Gamma(\Upsilon(4 S) \rightarrow X+\text { hadrons }) \sim E_{B} \log E_{B}
$$

and

$$
\mathcal{A}(B \rightarrow X K)=\left[\frac{Z_{00} m_{X}}{\pi^{3} m_{D} m_{D^{*}} a}\right]^{1 / 2} 2 c \Lambda p_{B} \cdot \epsilon_{X}^{*}
$$

\footnotetext{
${ }^{7}$ A subtlety associated with these scales will be discussed below.
} 

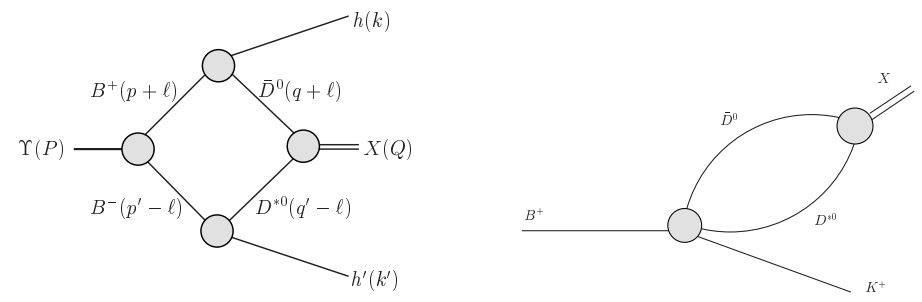

FIG. 13: $X$ Production from $\Upsilon(4 S)$ Decays via Coalescence (left) [76] and from $B$ Decays (right) [77].

for $X$ production in $B$ decays [77]. Here $\Lambda$ is an ultraviolet cutoff, and $c$ is a coupling defined by $\mathcal{A}\left(B \rightarrow D D^{*} K\right)=$ $c p_{B} \cdot \epsilon$. This expression was analysed further in Ref. 78 . where the authors set the cutoff to be $\Lambda=\Lambda_{\pi} \approx m_{\pi}$ which is a 'cross over momentum' scale defined by the transition from a phase space dominated invariant mass distribution to a resonance dominated invariant mass distribution. The improved estimate is then

$$
\operatorname{Br}\left(B^{+} \rightarrow X K^{+}\right)=2.7 \cdot 10^{-5}\left(\frac{\Lambda_{\pi}}{\pi}\right)^{1 / 2}\left(\frac{E_{B}}{0.5 \mathrm{MeV}}\right)^{1 / 2}
$$

Braaten and Kusunoki also obtained

$$
\frac{B r\left(B^{0} \rightarrow X K^{0}\right)}{B r\left(B^{+} \rightarrow X K^{+}\right)}=0-0.08
$$

(The upper end of this estimate agrees with the lower end of the molecular estimate to appear in Eq. 711)

Finally, Braaten and Kusunoki have used factorisation between universal long range behaviour and unknown short range behaviour to predict the binding energy dependence of decay rates [79, 80]. Using the results of Ref. [66] to fix the short range results, yields predictions such as

$$
\Gamma(X \rightarrow \pi \pi \pi J / \psi)=222 \mathrm{keV}\left(\frac{E_{B}+\Gamma_{X}^{2} / 16 E_{B}}{1 \mathrm{MeV}}\right)^{1 / 2}
$$

This method describes decays such as $X \rightarrow \pi \pi J / \psi$ via a virtual $\rho J / \psi$ channel and associates that channel with the scattering length $a$. It should be noted, however, that the presence of multiple channels generates multiple scattering lengths via the coupled channel weak binding relations

$$
E_{B}(\alpha)=\frac{1}{2 \mu_{\alpha} a_{\alpha}^{2}}
$$

where $\alpha$ denotes an $X$ channel and $E_{B}(\alpha)=m_{t o t}(\alpha)-m_{X}$. Thus, although the probability of channel $\alpha$ scales as $1 / a$, the dynamics associated with that channel scale with $a_{\alpha}=1 / \sqrt{2 \mu_{\alpha} E_{B}(\alpha)}$.

These scales can be seen clearly in the $X$ molecule model of the previous section. Numerically integrating the coupled channel Schrödinger equation and fitting the subsequent channel wavefunctions to the weak binding form (an example is shown in Fig. 14) yields estimates of the channel scattering lengths. For example, setting $\Lambda=1.13$ GeV gives a binding energy of $0.9 \mathrm{MeV}$ and hence $a_{D^{0} \bar{D}^{* 0}}=23.8 \mathrm{GeV}^{-1}$ while the fit to the numerically obtained wavefunction yields $a_{D^{0} \bar{D}^{* 0}}=24 \mathrm{GeV}^{-1}$. Similarly, for the $\rho J / \psi$ wavefunction one obtains a fit value of $a_{\rho \psi}=9.5$ $\mathrm{GeV}^{-1}$ while using $E_{B}(\rho \psi)=9.2 \mathrm{MeV}$ gives an expected value of $a_{\rho \psi}=9.3 \mathrm{GeV}^{-1}$. Similarly $a_{D^{-}} D^{*+}=7.6 \mathrm{GeV}^{-1}$. A selection of $X$ properties as a function of $\Lambda$ are given in Table VII Thus many scales are present when multiple channels contribute to the molecular structure of the $X$. As the binding energy decreases these additional channels disappear as $1 / a_{D^{0} \bar{D}^{* 0}}$ and the scale associated with each channel saturates at $a_{\alpha}=\left[2 \mu_{\alpha}\left(m_{\text {tot }}(\alpha)-m_{\text {tot }}\left(D^{0} \bar{D}^{* 0}\right)\right)\right]^{-1 / 2}$. At larger binding energies the interplay of these scales can complicate the description of $X$ dynamics and care must be exercised.

Notice that the weak binding wavefunction of Fig. 14 is quite accurate for distances greater than 2 fm. Similarly, the Fourier transformed weak binding wavefunction is accurate for momenta less than $200 \mathrm{MeV}$. Above this scale the wavefunction is quite small, hence the weak binding wavefunction may serve as a useful approximation to the full wavefunction in favourable circumstances. 
TABLE VII: $X$ Properties vs. Cutoff

\begin{tabular}{c|ccccc}
\hline$\Lambda(\mathrm{GeV})$ & $E_{B}(\mathrm{MeV})$ & $Z_{00}$ & $Z_{+-}$ & $Z_{\omega \psi}$ & $Z_{\rho \psi}$ \\
\hline \hline 1.14 & 1.0 & $89 \%$ & $4.0 \%$ & $5.0 \%$ & $0.7 \%$ \\
1.17 & 1.1 & $86 \%$ & $5.5 \%$ & $6.4 \%$ & $0.8 \%$ \\
1.20 & 1.4 & $82 \%$ & $7.5 \%$ & $8.1 \%$ & $0.8 \%$ \\
1.23 & 1.7 & $78 \%$ & $9.2 \%$ & $9.3 \%$ & $0.8 \%$ \\
1.30 & 2.7 & $69 \%$ & $14 \%$ & $12.7 \%$ & $0.7 \%$ \\
1.40 & 4.8 & $60 \%$ & $20 \%$ & $15 \%$ & $0.5 \%$ \\
1.50 & 8.0 & $55 \%$ & $24 \%$ & $16 \%$ & $0.3 \%$ \\
\hline \hline
\end{tabular}

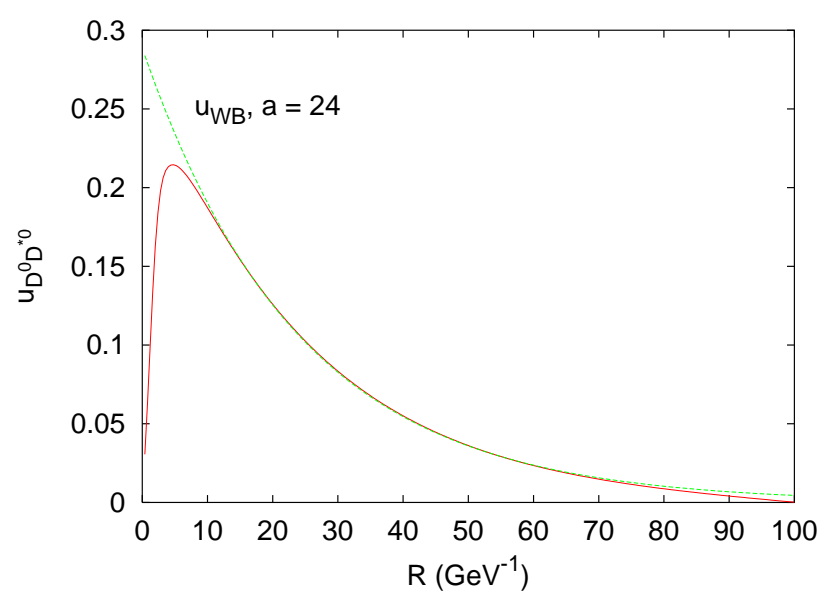

FIG. 14: The full $D_{0} D_{0}^{*}$ wavefunction and the weak binding wavefunction.

Finally, Al Fiky et al. 81] have improved the effective field theory of Braaten and Kusunoki (Eq. [57) by incorporating heavy quark and chiral symmetries. Thus the theory is written in terms of a superfield doublet combining the pseudoscalar and vector mesons:

$$
H_{a}^{(Q)}=\frac{1+\not 0}{2}\left[D_{a \mu}^{*(Q)} \gamma^{\mu}-D_{a}^{(Q)} \gamma_{5}\right], \quad \bar{H}^{(Q) a}=\gamma^{0} H_{a}^{(Q) \dagger} \gamma^{0}
$$

These fields have the usual transformation properties under heavy-quark spin symmetry and $\mathrm{SU}(2)_{V}$ flavor symmetry and describe heavy mesons with a definite velocity $v$. One then has $\mathcal{L}=\mathcal{L}_{2}+\mathcal{L}_{4}$ with

$$
\begin{aligned}
\mathcal{L}_{2} & =-i \operatorname{Tr}\left[\bar{H}^{(Q)} v \cdot \mathcal{D} H^{(Q)}\right]-\frac{1}{2 m_{P}} \operatorname{Tr}\left[\bar{H}^{(Q)} \mathcal{D}^{2} H^{(Q)}\right] \\
& +\frac{\lambda_{2}}{m_{P}} \operatorname{Tr}\left[\bar{H}^{(Q)} \sigma^{\mu \nu} H^{(Q)} \sigma_{\mu \nu}\right]+\frac{i g}{2} \operatorname{Tr} \bar{H}^{(Q)} H^{(Q)} \gamma_{\mu} \gamma_{5}\left[\xi^{\dagger} \partial^{\mu} \xi-\xi \partial^{\mu} \xi^{\dagger}\right]
\end{aligned}
$$

and

$$
-\mathcal{L}_{4}=\frac{C_{1}}{4} \operatorname{Tr}\left[\bar{H}^{(Q)} H^{(Q)} \gamma_{\mu}\right] \operatorname{Tr}\left[H^{(\bar{Q})} \bar{H}^{(\bar{Q})} \gamma^{\mu}\right]+\frac{C_{2}}{4} \operatorname{Tr}\left[\bar{H}^{(Q)} H^{(Q)} \gamma_{\mu} \gamma_{5}\right] \operatorname{Tr}\left[H^{(\bar{Q})} \bar{H}^{(\bar{Q})} \gamma^{\mu} \gamma_{5}\right]
$$

The covariant derivative is given by $\mathcal{D}_{a b}^{\mu}=\delta_{a b} \partial^{\mu}-(1 / 2)\left(\xi^{\dagger} \partial^{\mu} \xi+\xi \partial^{\mu} \xi^{\dagger}\right)_{a b}$ and $g$ is the $D^{*} D \pi$ coupling. The pion is realised nonlinearly as $\xi=\exp \left(i \pi \cdot \tau / \sqrt{2} f_{\pi}\right)$. The third term in Eq. 655 is needed to account for the $D-D^{*}$ mass difference $M\left(D^{*}\right)-M(D)=-2 \lambda_{2} / M(D)$.

Evaluating the traces yields for the $D \overline{D^{*}}$ sector

$$
\begin{aligned}
\mathcal{L}_{4, D D^{*}}= & -C_{1} D^{(Q) \dagger} D^{(Q)} D_{\mu}^{*(\bar{Q}) \dagger} D^{*(\bar{Q}) \mu}-C_{1} D_{\mu}^{*(Q) \dagger} D^{*(Q) \mu} D^{(\bar{Q}) \dagger} D^{(\bar{Q})} \\
& +C_{2} D^{(Q) \dagger} D_{\mu}^{*(Q)} D^{*(\bar{Q}) \dagger \mu} D^{(\bar{Q})}+C_{2} D_{\mu}^{*(Q) \dagger} D^{(Q)} D^{(\bar{Q}) \dagger} D^{*(\bar{Q}) \mu}+\ldots
\end{aligned}
$$


This lagrangian differs from that of Eq. 57 where the interaction is described in terms of a single parameter $\lambda_{0}$. This is related to the parameters above by $\lambda_{0}=-C_{1}=C_{2}$. The additional parameter in the lagrangian of Al Fiky et al. may be ascribed to distinguishing effective pseudoscalar and scalar exchange interactions between heavy mesons. Nevertheless, the binding energy of heavy pseudoscalar-vector states depends on a single linear combination of $C_{1}$ and $C_{2}$. The mass of the $X$ may be used to fix this linear combination and a prediction for a $B \bar{B}^{*}$ bound state at $10604 \mathrm{MeV}$ is obtained 81]. Predictions for possible $D \bar{D}, B \bar{B}, B \bar{D}^{*}$, and $D \bar{D}^{*}$ bound states are not possible until another linear combination of the couplings can be determined. Further discussion of other molecules is contained in Sect. IG

The mass of the $B \bar{B}^{*}$ molecule was obtained with the aid of the heavy quark mass scaling relation $C_{i} \sim 1 / m_{Q}$ which implies that $C_{i}\left(m_{b}\right)=m_{c} / m_{b} C_{i}\left(m_{c}\right)$. Furthermore, the pion exchange portion of the coupling constant (obtained upon integrating out the pions) is much smaller than the contact portion 82 . Both of these observations are not in agreement with the microscopic model quark+pion exchange model: pion exchange dominates the effective interaction and the pointlike interaction is controlled by scales such as the string tension, the light quark mass, and the pion mass.

The power and limitations of the effective field theory approach are easily seen. When enough data exists to determine coupling constants reliable predictions are possible in a broad range of problems. These predictions are under-pinned by QCD symmetries or universality. An example is the scaling of decay rates with binding energy shown in Eq. 62. However, the method fails when data is sufficiently sparse that parameters cannot be determined or multiple scales affect the dynamics.

\section{Microscopic Decay Models}

\section{Strong Decays}

The difficulties associated with predicting strong decays of mesons have already been discussed in Section IB 4 These difficulties are worse in molecular decays where uncertainties are compounded by poorly constrained structure and dynamics. There is, however, no utility in being too easily dissuaded! In this spirit three- and two-body decay processes driven by one gluon exchange are displayed in Figs. [15] and 16 respectively. The latter replace constituent mesons with kinematically allowed decay product mesons, either by rearrangement or by flavour changing processes. The former proceed via quark pair creation such as drives ${ }^{3} P_{0}$ or Cornell model meson decays. In the case of the $X$ molecular model of the previous section, dissociation gives rise to decays such as $X \rightarrow \rho J / \psi \rightarrow \pi \pi J / \psi$ or $X \rightarrow D \bar{D}^{*} \rightarrow D \bar{D} \gamma$
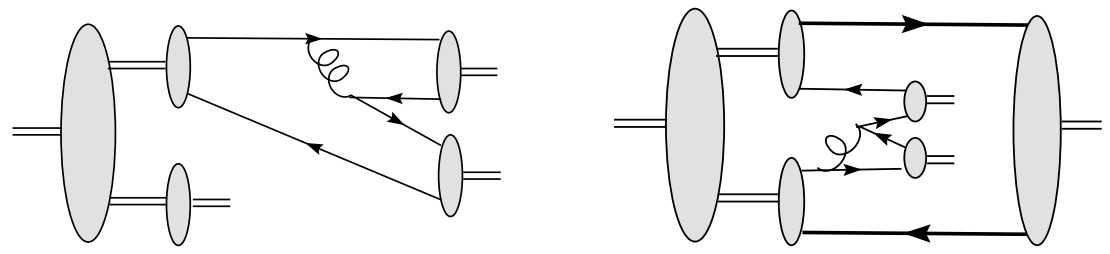

FIG. 15: $X$ Strong Decay via Dissociation (left) and $\pi \pi$ Production (right).
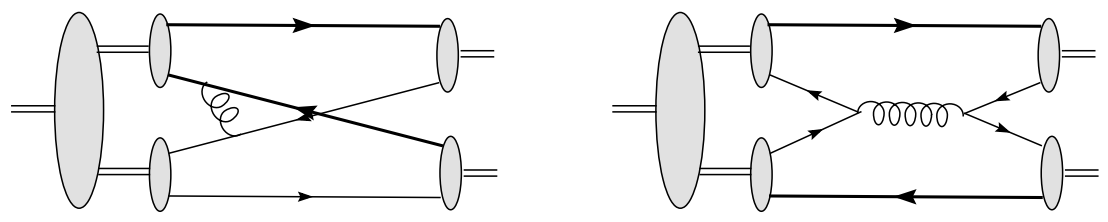

FIG. 16: $X$ Strong Decay via Rearrangement (left) and Annihilation (right).

In terms of the molecular $X$ model, the leading source of decays is dissociation. In the weak binding limit the decay rates are simple to estimate: to good approximation dissociation will proceed via the free space decay of the constituent mesons. Thus, for example,

$$
\Gamma(X \rightarrow \pi \pi J / \psi)=Z_{\rho \psi} \Gamma(\rho \rightarrow \pi \pi)
$$


and

$$
\Gamma\left(X \rightarrow D^{0} \bar{D}^{0} \pi^{0}\right)=2 Z_{00} \Gamma\left(D^{* 0} \rightarrow D^{0} \pi^{0}\right)
$$

The factor of two in the latter expression accounts for the S-wave combination $\frac{1}{\sqrt{2}}\left(D^{0} \bar{D}^{* 0}+\bar{D}^{0} D^{* 0}\right)$ in the bound state wavefunction. A more extensive list of predictions is given in Table VIII.

TABLE VIII: Some Decay Modes of the $X(3872)(\mathrm{keV})$.

\begin{tabular}{c|ccccccccc}
\hline$B_{E}(\mathrm{MeV})$ & $D^{0} \bar{D}^{0} \pi^{0}$ & $D^{0} \bar{D}^{0} \gamma$ & $D^{+} D^{-} \pi^{0}$ & $\left(D^{+} \bar{D}^{0} \pi^{-}+\mathrm{c} . \mathrm{c}\right) / \sqrt{2}$ & $D^{+} D^{-} \gamma$ & $\pi^{+} \pi^{-} J / \psi$ & $\pi^{+} \pi^{-} \gamma J / \psi$ & $\pi^{+} \pi^{-} \pi^{0} J / \psi$ & $\pi^{0} \gamma J / \psi$ \\
\hline \hline 0.7 & 67 & 38 & 5.1 & 4.7 & 0.2 & 1290 & 12.9 & 720 \\
1.0 & 66 & 36 & 6.4 & 5.8 & 0.3 & 1215 & 12.1 & 820 & 80 \\
\hline \hline
\end{tabular}

Strong decays in the case of strong binding are considerably more complicated. The formalism which describes this is discussed in Appendix A.

\section{Radiative Decays}

Radiative decays of molecules provide an interesting contrast to the impulse approximation processes which drive mesonic radiative decays. In the case of the $X$ radiative decay can occur via vector meson dominance (in the $\omega J / \psi$ and $\rho J / \psi$ components) or annihilation [42] ( $D \bar{D}^{*}$ components), as illustrated in Fig. 17. The vector meson dominance diagram will contribute to $X \rightarrow \gamma J / \psi$. It is clear that the amplitude must be proportional to the light vector meson wavefunction at the origin and to the $\hat{\chi}$ wavefunction for the channel in question. The specific result is

$$
\Gamma_{\mathrm{VMD}}=\frac{4}{27} \alpha \frac{q E_{\psi}}{m_{\chi}}\left|\psi_{\omega}(r=0)\right|^{2}\left(Z_{\omega \psi}^{1 / 2} \phi_{\omega \psi}(q)+3 Z_{\rho \psi}^{1 / 2} \phi_{\rho \psi}(q)\right)^{2}
$$

Decays to $\gamma \psi^{\prime}$ or $\gamma \psi_{2}$ can only proceed via annihilation and hence are very small.
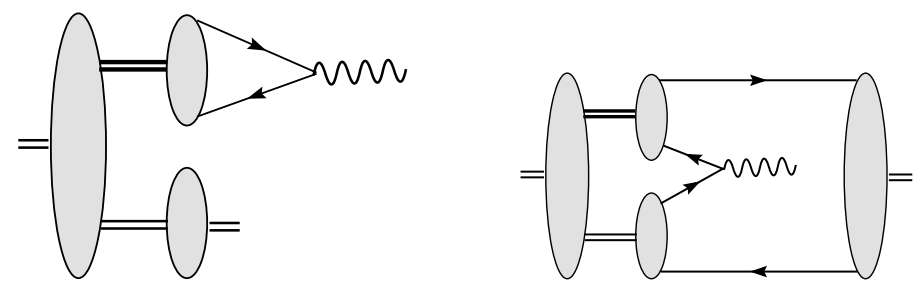

FIG. 17: X Radiative Decay via Vector Meson Dominance (left) and Annihilation (right) [42].

A variety of predictions are presented in Table IX Here, the fourth column summarises the results of Barnes and Godfrey [8] which are computed in the impulse, nonrelativistic, zero recoil, and dipole approximations. The results for $X \rightarrow \gamma J / \psi$ are particularly sensitive to model details - in fact this rate is identically zero for SHO wavefunctions. The sensitivity is examined in columns five and six which present the results of two additional computations. The first, labelled [A], employs the same approximations of Barnes and Godfrey but uses meson wavefunctions computed with a simple, but accurate, Coulomb+linear+smeared hyperfine potential. It is apparent that the $\gamma J / \psi$ rate is very sensitive to wavefunction details. Furthermore, one may legitimately question the use of the zero recoil and dipole approximations for the $\gamma J / \psi$ mode since the photon momentum is so large in this case. The sixth column (model [B]) dispenses with these approximations, and one finds a relatively large effect for $\gamma J / \psi$.

Table IX makes it clear that computations of the $\gamma J / \psi$ radiative transition of the $\chi_{c 1}^{\prime}$ are very sensitive to model details $^{8}$. The result of Barnes and Godfrey is similar to that computed here for the molecular $\hat{\chi}$ state but is much smaller than models A and B. Furthermore, the rates for $\gamma \psi^{\prime \prime}$ and $\gamma \psi_{2}$ are very small for a molecular $X$ (at the

\footnotetext{
8 There is an additional error induced by arbitrarily changing the quark model $\chi^{\prime}$ mass to $3872 \mathrm{MeV}$. This is made clear through the observation that the dipole formula for the width scales as $q^{3}$ whereas the momentum space formula scales as $q$.
} 
order of $\mathrm{eV}$ ) and quite small for a charmonium $X$. Perhaps the most robust diagnostic is the $\gamma \psi^{\prime}$ decay mode. For a molecular $\hat{\chi}$ this can only proceed via the annihilation diagram and hence is very small. Clearly a measurement of the $\gamma J / \psi$ and $\gamma \psi^{\prime}$ decay modes of the $X(3872)$ will provide compelling clues to its internal structure.

TABLE IX: E1 Decays of the X(3872) 42].

\begin{tabular}{ccc|ccc|c}
\hline mode & $m_{f}(\mathrm{MeV})$ & $q(\mathrm{MeV})$ & $\Gamma[c \bar{c}](\mathrm{keV})$ & $\Gamma[c \bar{c}](\mathrm{keV})$ & $\Gamma[c \bar{c}](\mathrm{keV})$ & $\Gamma\left[\hat{\chi}_{c 1}\right](\mathrm{keV})$ \\
& & & {$[\mathrm{B} \& \mathrm{G}]$} & {$[\mathrm{A}]$} & {$[\mathrm{B}]$} & \\
\hline \hline$\gamma J / \psi$ & 3097 & 697 & 11 & 71 & 139 & 8 \\
$\gamma \psi^{\prime}\left(2^{3} S_{1}\right)$ & 3686 & 182 & 64 & 95 & 94 & 0.03 \\
$\gamma \psi^{\prime \prime}\left(1^{3} D_{1}\right)$ & 3770 & 101 & 3.7 & 6.5 & 6.4 & 0 \\
$\gamma \psi_{2}\left(1^{3} D_{2}\right)$ & 3838 & 34 & 0.5 & 0.7 & 0.7 & 0 \\
\hline \hline
\end{tabular}

\section{E. New Data and Further Analysis}

The apparent success of the molecular interpretation of the $X$ has been challenged by several recent experimental results. These are reviewed here.

- The ratio of production of the $X$ in neutral and charged $B$ decays (Eq. 30) lies in the range 0.25 - 0.97 with $64 \%$ C.L. and hence is rather large compared to expectations based on the molecular picture. This ratio may be estimated with the diagrams shown in Fig. 18. The colour leading diagram (left panel) permits the processes $B^{+} \rightarrow K^{+} D^{0} \bar{D}^{* 0}$ which will contribute to $K^{+} X, B^{+} \rightarrow K^{0} \bar{D}^{0} D^{*+}$ which tests $X^{+}$production and cusp effects, $B^{0} \rightarrow K^{0} D^{-} D^{*+}$ which accesses the $X$ through the suppressed charged component, and $B^{0} \rightarrow K^{+} D^{-} D^{* 0}$ which tests $X^{-}$production and cusp effects. The colour suppressed diagram (right panel) permits the following decays: $B^{+} \rightarrow K^{+} D^{0} \bar{D}^{* 0}$, $B^{+} \rightarrow K^{+} D^{+} D^{*-}, B^{0} \rightarrow K^{0} D^{0} \bar{D}^{* 0}$, and $B^{0} \rightarrow K^{0} D^{+} D^{*-}$. Since the diagrams involve identical mesons (at the strong level) and only differ by topology one may predict the ratio

$$
\frac{B r\left(B^{0} \rightarrow K^{0} X\right)}{B r\left(B^{+} \rightarrow K^{+} X\right)}=\frac{\left|4 Z_{+-}^{1 / 2}+Z_{00}^{1 / 2}\right|^{2}}{\left|4 Z_{00}^{1 / 2}+Z_{+-}^{1 / 2}\right|^{2}} \approx 0.06-0.29
$$

The lower estimate assumes that there is no relative phase between the topologies (it is difficult to imagine why there should be one) and that the $X$ is completely dominated by the neutral $D \bar{D}^{*}$ component. The higher estimate assumes that the neutral component is $90 \%$ of the $X$ while the charged is $10 \%{ }^{9}$. Given the large errors in the experimental result of Eq. [30, it is too early to rule out the molecular interpretation of this state. An accurate determination of this ratio will measure the relative importance of the suppressed $D^{+} D^{*-}$ component of the $X$.
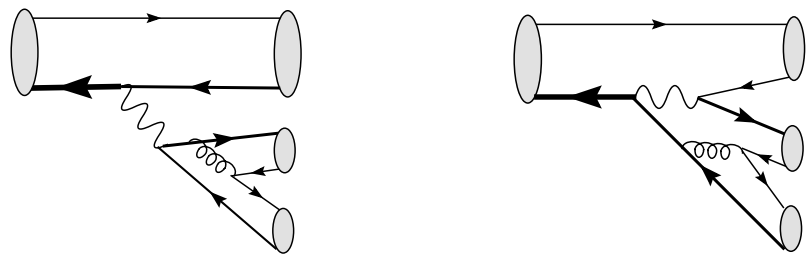

FIG. 18: $B \rightarrow K X$. Colour enhanced amplitude (left). Colour suppressed amplitude (right).

- Eqs. 34, 35, and 36] imply that

$$
R=\frac{B r\left(X \rightarrow D^{0} \bar{D}^{0} \pi^{0}\right)}{B r\left(X \rightarrow \pi^{+} \pi^{-} J / \psi\right)}=22 \pm 13
$$

This should be contrasted with an earlier measurement from Belle which determined 83

\footnotetext{
${ }^{9}$ Assuming a negative relative phase raises this estimate to $0.25-0.51$.
} 


$$
\operatorname{Br}\left(B^{+} \rightarrow X K^{+}\right) \operatorname{Br}\left(X \rightarrow D^{0} \bar{D}^{0} \pi^{0}\right)<6 \cdot 10^{-5}
$$

at $90 \%$ C.L. Eqs. 34 and 35 then imply that $R<6$. A reliable determination of this ratio will be crucial to understanding the $X$ and further experimental effort is encouraged.

In the molecular picture $R$ is driven by decays $D^{* 0} \rightarrow D^{0} \pi^{0}$ and $\rho \rightarrow \pi \pi$ and hence is given (in the weak binding limit) by

$$
R=\frac{2 Z_{00} \Gamma\left(D^{* 0}\right)}{Z_{\rho \psi} \Gamma(\rho)}
$$

With the pion and quark dynamics assumed in Section IIB 4 one obtains $Z_{00} \approx 0.8$ and $Z_{\rho \psi} \approx 0.01$ at a binding energy of $0.7 \mathrm{MeV}$. Thus $R \approx 0.08$, clearly in conflict with experiment.

If the $X$ is indeed a weakly bound molecule, Eq. 74 must be accurate and hence the only way to reconcile this prediction with the data is if the $\rho J / \psi$ component has been severely overestimated. The required probability is $Z_{\rho \psi} \approx 0.007 \%$.

- The large rate for $D^{0} \bar{D}^{0} \pi^{0}$ implies that $B r(X \rightarrow \pi \pi J / \psi)<0.05$. With Eqs. 34] 35 this implies that

$$
\operatorname{Br}(B \rightarrow K X)>2 \cdot 10^{-4}
$$

which is comparable to the branching ratio for $\chi_{c 1}: \operatorname{Br}\left(B \rightarrow \chi_{c 1} K\right)=4.0(1.0) \cdot 10^{-4}[17$ ]. However, both of these results are very close to bounds established by BaBar 39, 40, 84]:

$$
\operatorname{Br}\left(B^{ \pm} \rightarrow K^{ \pm} X\right)<3.2 \cdot 10^{-4}(90 \% \text { C.L. })
$$

and

$$
\operatorname{Br}(X \rightarrow \pi \pi J / \psi)>4.2 \% .
$$

These results, and the conflict with the earlier Belle result (Eq. 73), can be most easily interpreteted by assuming that the rate to $D^{0} \bar{D}^{0} \pi^{0}$ has been overestimated in Eq. 72

If a large $D^{0} \bar{D}^{0} \pi^{0}$ decay mode is confirmed, the simplest interpretation is that the $X$ contains substantial $c \bar{c}-$ in keeping with the production characteristics discussed at the end of Sect. ПIA More specifically the $X$ must mix strongly with the $\chi_{c 1}^{\prime}$. This may be estimated with the aid of the ${ }^{3} P_{0}$ model and Fig. 19] The net result is a mixing matrix element

$$
a_{\chi}=\sqrt{2} Z_{00}^{1 / 2} \int d^{3} k \psi_{X}(k) \mathcal{A}(-k)
$$

where $\mathcal{A}$ is the ${ }^{3} P_{0}$ amplitude for $\chi_{c 1}^{\prime} \rightarrow D^{0} \bar{D}^{* 0}$. Here $\psi_{X}(k)=(\pi \sqrt{a})^{-1}\left(k^{2}+a^{-2}\right)^{-1}$ is the weak binding $X$ wavefunction.

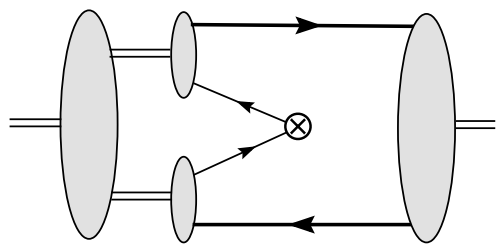

FIG. 19: $X-\chi_{c 1}$ Mixing.

Evaluating the integral yields surprisingly large mixing matrix elements, which are summarised in Table $\mathbf{X}$ As can be seen, the mixing amplitude is diminished as the $X$ scattering length gets larger. Surprisingly, mixing with the excited $\chi_{c 1}^{\prime}$ is not strongly suppressed with respect to the ground state $\chi_{c 1}$. Most surprisingly, the mixing matrix element can be quite large, so large that the relatively close $\chi_{c 1}^{\prime}(3930)$ has a very (nonperturbatively) large mixing amplitude with the $X$. This unexpected result explains the large $K X$ branching ratio in $B$ decays and the $X$ 
TABLE X: $X-\chi_{c 1}$ Mixing.

\begin{tabular}{cccccc}
\hline state & $E_{B}(\mathrm{MeV})$ & $a(\mathrm{fm})$ & $Z_{00}$ & $a_{\chi}(\mathrm{MeV})$ & prob \\
\hline \hline$\chi_{c 1}$ & 0.1 & 14.4 & $93 \%$ & 94 & $5 \%$ \\
& 0.5 & 6.4 & $83 \%$ & 120 & $10 \%$ \\
$\chi_{c 1}^{\prime}$ & 0.1 & 14.4 & $93 \%$ & 60 & $100 \%$ \\
& 0.5 & 6.4 & $83 \%$ & 80 & $>100 \%$ \\
\hline \hline
\end{tabular}

production characteristics in $p \bar{p}$ collisions. The complete phenomenological implications of this result have not been explored 85$]$.

- Belle have found the $\gamma J / \psi$ decay mode of the $X$ and report a large branching of $14 \%$ of the $\pi \pi J / \psi$ mode (see Eq. (32). In the model of Sect. [ID one expects this to be $0.4 \%$ at a binding energy of $0.7 \mathrm{MeV}-$ much too small to explain the data. Note, however, that the predicted rate for $\chi_{c 1}^{\prime} \rightarrow \gamma J / \psi$ is $10-140 \mathrm{keV}$ (Table IX]; the upper end of this prediction implies a branching ratio of $14 \%$ if the $\pi \pi J / \psi$ mode has a width of roughly $1 \mathrm{MeV}$. But this implies that the $X$ has a very large $\chi_{c 1}^{\prime}$ component or that the predicted $\pi \pi J / \psi$ rate is too large. In view of the previous discussion, both of these possibilities appear likely.

\section{F. Summary}

It is possible that all of the new experimental data may be explained if the coupling of the $X$ to the $\chi_{c 1}^{\prime}$ is correctly incorporated in the coupled channel formalism. This would increase the $\gamma J / \psi$ branching fraction, permit the large $D^{0} \bar{D}^{0} \pi^{0}$ decay mode, and allow the charmonium-like production characteristics. A detailed phenomenology of this scenario remains to be constructed.

Note that cusp, tetraquark, renormalised $\chi_{c 1}^{\prime}$, virtual state, and molecular interpretations all assume that the $X$ is dominantly (or heavily modified by) a $c \bar{c} u \bar{u}$ Fock space component. The chief difference among the models is the spatial, colour, and spin configuration of the state, which is of course, driven by different assumed dynamics. Thus distinguishing these models is a qualitative issue and choosing between them is question of efficiency of description and consistency of adjunct predictions.

Finally, we comment on two misapprehensions in $X$ dynamics that have appeared in the literature. First, the $3 \pi J / \psi$ decay mode proceeds via the $\omega J / \psi$ component of the $X$. It is sometimes thought that the decay occurs via the natural line width of the $\omega$. Since the width of $\omega$ is only $8 \mathrm{MeV}$ and the $\omega J / \psi$ system is $7.5 \mathrm{MeV}$ above the $X$ mass, the probability of the $X(\omega J / \psi)$ fluctuating into $3 \pi J / \psi$ is very small. Thus the large relative branching fraction for the $3 \pi$ mode cannot be correct. This argument is incorrect: the $\omega J / \psi$ component of the $X$ is off-shell due to the dynamics leading to binding and the $\omega$ need not fluctuate to a "lower mass" to permit decay.

Second, Suzuki [86] has argued that the one-pion-exchange potential as described in Sect. IIB4] is not valid because the process $D^{*} \rightarrow D \pi$ can occur on-shell. This argument is incorrect. The four point function that describes $D \bar{D}^{*}$ scattering does indeed exhibit a pole (as opposed to the $N N$ interaction) due to set of measure zero in the phase space available to the $D \bar{D} \pi$ system. The pole induces a small imaginary part to the scattering amplitude, which describes the decay $X \rightarrow D \bar{D} \pi$. Of course, this decay may be separately estimated in perturbation theory as in Sect. IID The real part of the scattering amplitude remains unchanged and its analysis may proceed as for $N N$ scattering. The procedure is described in more detail in Appendix B.

\section{G. Other Molecules}

Other heavy molecular states can be generated from the one pion exchange mechanism discussed above. Effective potentials in the various channels are discussed in Appendix B. Isovector channels never bind; some properties of isoscalar channels which bind are listed in Table XI These results have been computed with $\Lambda=1.23 \mathrm{GeV}$ and generally agree with Törnqvist [61] except that I find only one $D^{*} \bar{D}^{*}$ bound state with $J^{P C}=0^{++}$.

Note that binding energies in the $B B$ channels are quite large. In particular, the $1^{++}\left(B \bar{B}^{*}\right)$ has a binding energy of $43 \mathrm{MeV}$, in contrast to the binding energy of $0.2 \mathrm{MeV}$ predicted by Al Fiky et al. [81, 82] (see Sect. [IC). The latter is predicated upon an effective field theory description of $H \bar{H}^{*}$ interactions and the scaling relation $C_{i} \sim 1 / m_{Q}$. This is quite different from one pion exchange and quark exchange interactions which do not scale with $m_{Q}$. Thus there is a conflict between these approaches (where in principle, none should exist) and further effort is required to resolve this discrepancy. Certainly, one expects that the $B \bar{B}^{*}$ system should be more strongly bound than $D \bar{D}^{*}$ since the kinetic energy is greatly reduced - in agreement with the pion exchange model predictions. 
TABLE XI: Isoscalar Heavy Quark Molecules

\begin{tabular}{ccccc}
\hline state & $J^{P C}$ & channels & mass $(\mathrm{MeV})$ & $E_{B}$ \\
\hline \hline$D^{*} \bar{D}^{*}$ & $0^{++}$ & ${ }^{1} S_{0},{ }^{5} D_{0}$ & 4019 & 1.0 \\
$B \bar{B}^{*}$ & $0^{-+}$ & ${ }^{3} P_{0}$ & 10543 & 61 \\
$B \bar{B}^{*}$ & $1^{++}$ & ${ }^{3} S_{1},{ }^{3} D_{1}$ & 10561 & 43 \\
$B^{*} \bar{B}^{*}$ & $0^{++}$ & ${ }^{1} S_{0},{ }^{5} D_{0}$ & 10579 & 71 \\
$B^{*} \bar{B}^{*}$ & $0^{-+}$ & ${ }^{3} P_{0}$ & 10588 & 62 \\
$B^{*} \bar{B}^{*}$ & $1^{+-}$ & ${ }^{3} S_{1},{ }^{3} D_{1}$ & 10606 & 44 \\
$B^{*} \bar{B}^{*}$ & $2^{++}{ }^{1} D_{2},{ }^{5} S_{2},{ }^{5} D_{2},{ }^{5} G_{2}$ & 10600 & 50 \\
\hline \hline
\end{tabular}

The molecular $\hat{\chi}_{c 0}$ (the $J^{P C}=0^{++} D^{*} \bar{D}^{*}$ state) is interesting because it may be accessible in B factories and at Fermilab. This state can decay to $\omega J / \psi, \eta \eta_{c}$, and $\eta^{\prime} \eta_{c}$. A rough computation of the partial widths yields

$$
\Gamma\left(\hat{\chi}_{c 0} \rightarrow \omega J / \psi\right) \approx 200 \mathrm{keV}
$$

and

$$
\Gamma\left(\hat{\chi}_{c 0} \rightarrow \eta \eta_{c}\right) \approx 600 \mathrm{keV} .
$$

Finally, unlike the case of the $X$, no nearby hidden flavour meson-meson channels exist for these states. Thus their short range structure is expected to be substantially simpler than that of the $X(3872)$. 


\title{
III. OTHER NEW CHARMONIA
}

\author{
A. $h_{c}$
}

The $h_{c}$ has been observed by CLEO 87 in the reaction $\psi(2 S) \rightarrow \pi^{0} h_{c} \rightarrow(\gamma \gamma)\left(\gamma \eta_{c}\right)$ with a mass of $3524.4 \pm 0.6 \pm 0.4$ $\mathrm{MeV}$ at a significance greater than $5 \sigma$. The observation has been made in exclusive measurements where the $\eta_{c}$ has been reconstructed, and in inclusive measurements (see Fig 20). The mass may be compared to the quark model predictions listed in Table Which range from 3516 to $3526 \mathrm{MeV}$ (or $3474 \mathrm{MeV}$ for quenched lattice computations). Furthermore, the measured product of branching fractions

$$
\operatorname{Br}\left(\psi(2 S) \rightarrow \pi^{0} h_{c}\right) \operatorname{Br}\left(h_{c} \rightarrow \gamma \eta_{c}\right)=(4.0 \pm 0.8 \pm 0.7) \cdot 10^{-4}
$$

is in close agreement with a theoretical estimate of $3.8 \cdot 10^{-4}$ of Ref. 88 ]. (This reference may also be consulted for an extensive discussion of spin splittings in quark models.) Thus it appears that the $h_{c}$ is conforming to expectations.

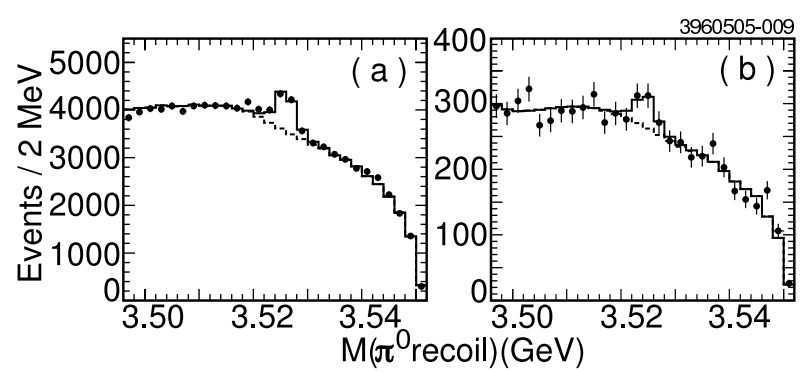

FIG. 20: Inclusive Production of the $h_{c}$ in $\psi(2 S) \rightarrow \pi^{0} X$. Monte Carlo (left); Data (right) [87].

Nevertheless, as discussed in Section [B] the $h_{c}$ is an important diagnostic for the spin-dependent structure of the quark-quark interaction. The fact that it lies within $2 \mathrm{MeV}$ of the spin-averaged mass of the $\chi_{c J}$ multiplet: $M_{\text {c.o.g. }}=\frac{1}{9}\left(\chi_{0}+5 \chi_{1}+9 \chi_{2}\right)=3525.36$ implies that the long range spin-spin interaction $V_{4}$ is very small, in agreement with the effective scalar confinement picture.

\section{B. $\eta_{c}^{\prime}$}

As discussed above, hyperfine splittings are a useful diagnostic of spin-dependent confinement interactions. An old result from Crystal Ball [89] caused some consternation since it implied a rather large spin splitting in the radial $\psi(2 S)-\eta_{c}(2 S)$ system. The situation has recently changed with new measurements from Belle, CLEO, and BaBar.

Belle [90] have observed an $\eta_{c}^{\prime}$ candidate in $39 \pm 11$ events in the $K_{S} K \pi$ system of the decay $B \rightarrow K K_{S} K^{-} \pi^{+}$. The measured mass of the resonance is $3654 \pm 6 \pm 8 \mathrm{MeV}$ and the width was determined to be $\Gamma=15_{-15}^{+24} \mathrm{MeV}(\Gamma<55$ $\mathrm{MeV}$ at $90 \%$ C.L.).

Belle also measured the ratio of branching fractions

$$
\frac{B r\left(B \rightarrow K \eta_{c}^{\prime}\right) B r\left(\eta_{c}^{\prime} \rightarrow K_{S} K^{-} \pi^{+}\right)}{\operatorname{Br}\left(B \rightarrow K \eta_{c}\right) \operatorname{Br}\left(\eta_{c} \rightarrow K_{S} K^{-} \pi^{+}\right)}=0.38 \pm \pm 0.12 \pm 0.05
$$

Assuming equal branching ratios for $\eta_{c}$ and $\eta_{c}^{\prime}$ to $K_{S} K \pi$ (this assumption is discussed in Ref. 91]) gives $B r(B \rightarrow$ $\left.K \eta_{c}^{\prime}\right) / \operatorname{Br}\left(B \rightarrow K \eta_{c}\right)=0.38$. This may be compared to the factorisation expression:

$$
\frac{B r\left(B \rightarrow K \eta_{c}^{\prime}\right)}{B r\left(B \rightarrow K \eta_{c}\right)}=\frac{p_{f}\left(\eta_{c}^{\prime}\right)}{p_{f}\left(\eta_{c}\right)} \cdot \frac{f_{\eta_{c}^{\prime}}^{2}}{f_{\eta_{c}}^{2}} \cdot \frac{\left|\xi\left(w\left(\eta_{c}^{\prime}\right)\right)\right|^{2}}{\left|\xi\left(w\left(\eta_{c}\right)\right)\right|^{2}} \cdot \frac{\left[\left(m_{B}+m_{K}\right)^{2}-m_{\eta_{c}^{\prime}}^{2}\right]^{2}}{\left[\left(m_{B}+m_{K}\right)^{2}-m_{\eta_{c}}^{2}\right]^{2}}
$$

where $w=v \cdot v^{\prime}=\left(m_{B}^{2}+m_{K}^{2}-m_{\eta}^{2}\right) /\left(2 m_{B} m_{K}\right)$.

A quark model computation gives 92 


$$
f_{\eta_{c}}=424 \mathrm{MeV}
$$

and

$$
f_{\eta_{c}^{\prime}}=243 \mathrm{MeV}
$$

And, using a simple model for the Isgur-Wise form factor $\xi(w)=(2 /(1+w))^{2}$ gives

$$
\frac{B r\left(B \rightarrow K \eta_{c}^{\prime}\right)}{B r\left(B \rightarrow K \eta_{c}\right)}=0.38
$$

in agreement with the data and the assumed equality of branching ratios.

More recently, the CLEO collaboration 93] have observed the $\eta_{c}^{\prime}$ in $\gamma \gamma \rightarrow \eta_{c}^{\prime} \rightarrow K_{S} K^{ \pm} \pi^{\mp}$ (Fig. 21] right panel) and find a Breit-Wigner mass and width of $3642.9 \pm 3.1 \pm 1.5 \mathrm{MeV}$ and $\Gamma=6.3 \pm 12.4 \pm 4.0 \mathrm{MeV}$ respectively. Assuming equal $K_{S} K \pi$ branching fractions as above, they also obtain

$$
\Gamma\left(\eta_{c}^{\prime} \rightarrow \gamma \gamma\right)=1.3 \pm 0.6 \mathrm{keV}
$$

Finally, BaBar 94 have observed an $\eta_{c}^{\prime}$ candidate in $112 \pm 24$ events in $\gamma \gamma \rightarrow \eta_{c}^{\prime} \rightarrow K_{S} K \pi$ (Fig. 21 left panel). They fit a mass of $3630.8 \pm 3.4 \pm 1.0 \mathrm{MeV}$ and obtain a width of $\Gamma=17.0 \pm 8.3 \pm 2.5 \mathrm{MeV}$. The angular distribution of the decay products and of the total transverse momentum both suggest an assignment of $J^{P C}=0^{-+}$or $J>2$, with the latter being unlikely for such a light state.
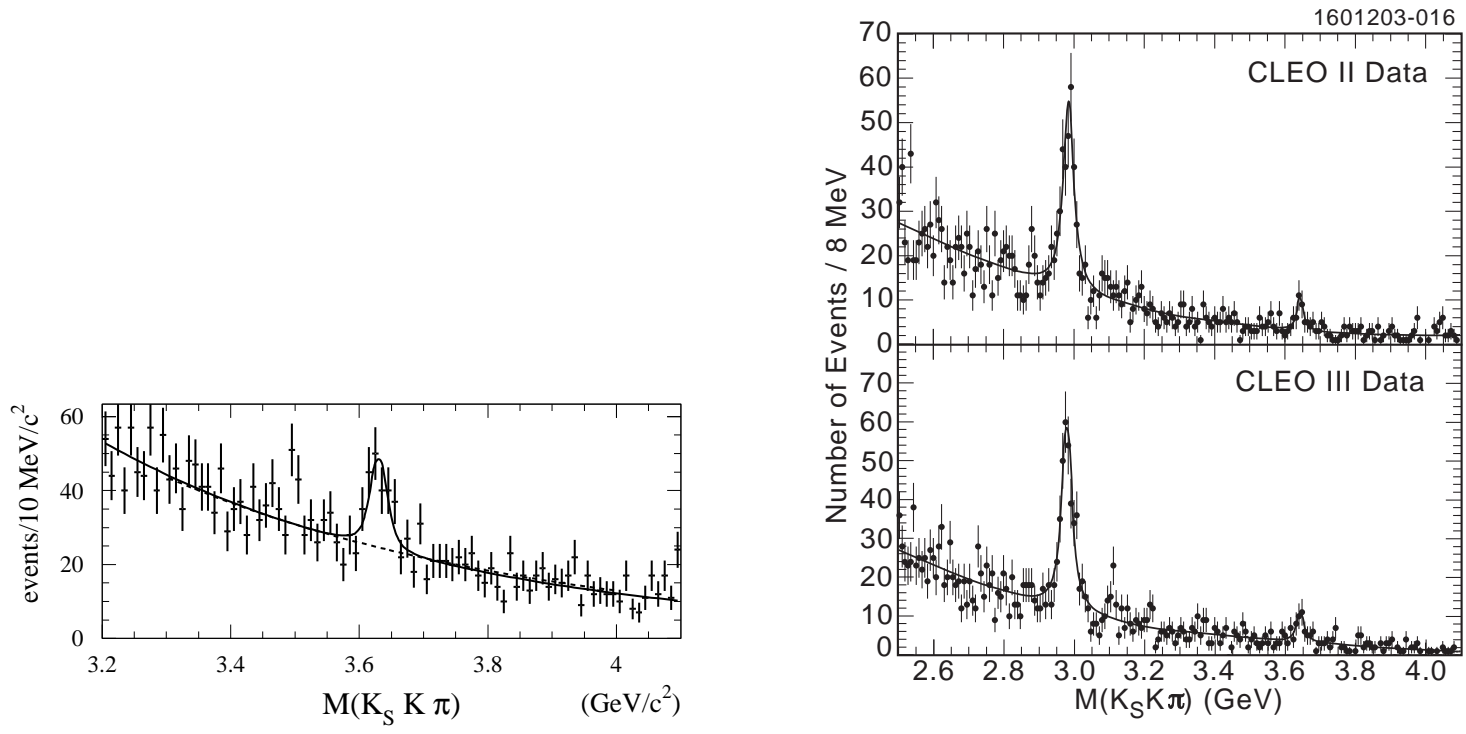

FIG. 21: Fit $\eta_{c}(2 S)$ by BaBar[94](left) and CLEO 93] (right).

As mentioned above, the $\eta_{c}^{\prime}$ is of some interest because its splitting with the $\psi^{\prime}$ is driven by the hyperfine interaction and hence probes this interaction in a new region. Specifically, the ground state vector-pseudoscalar splitting is

$$
M(J / \psi)-M\left(\eta_{c}\right)=117 \mathrm{MeV}
$$

whereas the excited splitting is now measured to be

$$
M\left(\psi^{\prime}\right)-M\left(\eta_{c}^{\prime}\right)=48 \mathrm{MeV}
$$

Theoretical expectations for the former range from 108 to $123 \mathrm{MeV}$ in simple (or 'relativised') quark models (see Table III) and thus are within expectations. Alternatively, the latter is predicted to be $67 \mathrm{MeV}$ in the quark model 
of Eichten, Lane, and Quigg [95]. However, the authors note that including unquenching effects due to open charm meson loops lowers this splitting to $46 \mathrm{MeV}$ which is taken as evidence in favour of their 'unquenched' quark model. However, the simple quark models mentioned above 13. find splittings of $42-53 \mathrm{MeV}$, indicating that it is too early to make definitive conclusions about loop effects. It is worth noting, furthermore, that attempts to unquench the quark model are fraught with technical difficulty (see Section IB 4) and a great deal of effort is required before we can be confident in the results of any model.

\section{C. $X(3940)$}

The $X(3940)$ is seen by Belle 96 recoiling against $J / \psi$ in $e^{+} e^{-}$collisions. The state has a Breit-Wigner mass of $3943 \pm 6 \pm 6 \mathrm{MeV}$ and a width of less than $52 \mathrm{MeV}$ at $90 \%$ C.L. [97]. The $X$ is seen to decay to $D \bar{D}^{*}$ and not to $\omega J / \psi$ or $D \bar{D}$.

It is natural to attempt a $2 P c \bar{c}$ assignment for this state since the expected mass of the $2^{3} P_{J}$ multiplet is 3850 - $3980 \mathrm{MeV}$ (see Table III) and the expected widths are $20-130 \mathrm{MeV}$ (see Table IV). Indeed, if the $D \bar{D}^{*}$ mode is dominant it suggests that the $X(3940)$ is the $\chi_{c 1}^{\prime}$. There is, however, a problem with this assignment. Fig. 22 shows the recoil mass spectrum up to $4.5 \mathrm{GeV}$. The bumps visible in the figure are a $3.3 \sigma$ fluctuation at $2500 \mathrm{MeV}^{10}$, the $\eta_{c}$ at $2980 \mathrm{MeV}$, a structure usually associated with the $\chi_{c 0}(3415)$ (but probably due to $\chi_{c 0}, \chi_{c 1}$, and $\chi_{c 2}$ ), the $\eta_{c}^{\prime}$ at 3638 , and the $X(3940)$. Thus there is no clear evidence for the $\chi_{c 1}$, and one may suspect that the $\chi_{c 1}^{\prime}$ should also not be a strong signal in this reaction. This has led to speculation that the $X(3940)$ is the radially excited $\eta_{c}^{\prime \prime}\left[98\right.$, 99]. Unfortunately this interpretation also has its problems as the expected mass of the $\eta_{c}^{\prime \prime}$ is $4040-4060 \mathrm{MeV}$, approximately $100 \mathrm{MeV}$ too high. An angular analysis of the decay products of the $X$ will help resolve this issue.

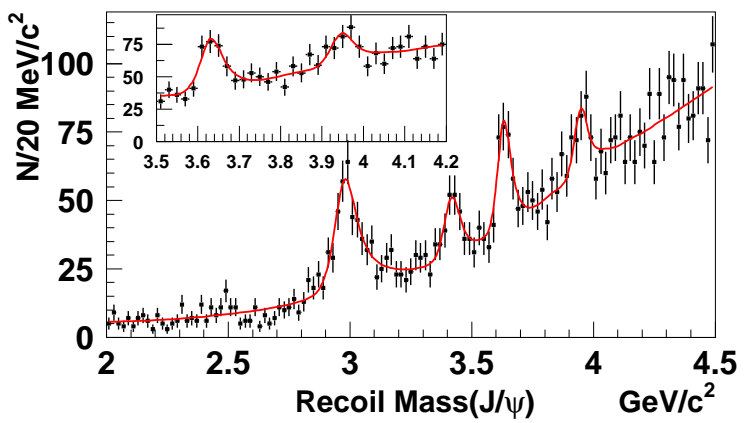

FIG. 22: $X$ (3940) Signal in $e^{+} e^{-} \rightarrow J / \psi+$ charm. Ref. 96].

An interesting addendum to the issues surrounding the $X(3940)$ concerns attempts to compute the production of the various charmonium states. Table XII displays the predicted cross sections from two NRQCD computations and experimental results from Belle and BaBar. It appears that NRQCD is under-predicting $\eta_{c} J / \psi$ production cross sections by an order of magnitude. However Bondar and Chernyak 103 have argued that NRQCD is not applicable to processes such as $e^{+} e^{-} \rightarrow J / \psi \eta_{c}$ because the charm quark is not sufficiently heavy. Using general methods for hard exclusive processes and a model $J / \psi$ light cone wavefunction gives the result labelled as BC in Table XII The last line refers to a light cone computation 104 which also produces much larger cross sections than NRQCD. The authors also not that NLO corrections can be large.

TABLE XII: Cross Sections (fb) for $e^{+} e^{-} \rightarrow J / \psi H$ at $\sqrt{s}=10.6 \mathrm{GeV}$.

10 Could this be the tensor glueball? 


\begin{tabular}{lccc}
\hline$H$ & $\eta_{c}$ & $\chi_{c 0}$ & $\eta_{c}^{\prime}$ \\
\hline \hline BaBar[39] & $17.6 \pm 2.8 \pm 2.1$ & $10.3 \pm 2.5 \pm 1.8$ & $16.4 \pm 3.7 \pm 3.0$ \\
Belle[100] & $25.6 \pm 2.8 \pm 3.4$ & $6.4 \pm 1.7 \pm 1.0$ & $16.5 \pm 3.0 \pm 2.4$ \\
\hline BL[101] & $2.31 \pm 1.09$ & $2.28 \pm 1.03$ & $0.96 \pm 0.45$ \\
LHC[102] & 5.5 & 6.9 & 3.7 \\
BC[103] & $\sim 33$ & & \\
BLL[104] & 26.7 & & 26.6 \\
\hline \hline
\end{tabular}

D. $Y(3940)$

Belle claim the discovery 105 of a second resonance at $3940 \mathrm{MeV}$, this time in the $\omega J / \psi$ subsystem of the process $B \rightarrow K \pi \pi \pi J / \psi$. The S-wave Breit Wigner mass and width are (see Fig. 23) determined to be $3943 \pm 11 \pm 13 \mathrm{MeV}$ and $87 \pm 22 \pm 26 \mathrm{MeV}$ respectively. A total of $58 \pm 11$ events are claimed for the $Y$ with a significance of $8 \sigma$. The state has not been seen in the decay modes $Y \rightarrow D \bar{D}$ or $D \bar{D}^{*}$. Because it does not share the production or decay channels of the $X(3940)$ it is possible that these states are distinct.

As for the $X(3940)$, the mass and width of the $Y$ suggest a radially excited $\mathrm{P}$-wave charmonium. However, the $\omega J / \psi$ decay mode is peculiar. In more detail, Belle measure

$$
\operatorname{Br}(B \rightarrow K Y) \operatorname{Br}(Y \rightarrow \omega J / \psi)=(7.1 \pm 1.3 \pm 3.1) \cdot 10^{-5} .
$$

One expects that the branching ratio of a radially excited $\chi_{c J}$ is less than that of the ground state $\chi_{c J}: \operatorname{Br}(B \rightarrow$ $\left.K \chi_{c J}^{\prime}\right)<\operatorname{Br}\left(B \rightarrow K \chi_{c J}\right)=4(1) \cdot 10^{-4}[17]$. Thus $\operatorname{Br}(Y \rightarrow \omega J / \psi)>17 \%$, which is unusual for a canonical $c \bar{c}$ state above open charm threshold.

Thus the $Y$ is something of an enigma, driving the observation of the Belle collaboration that it is perhaps a hybrid. The unusual decay mode and branching fraction are consistent with the claims that a hybrid should have strongly suppressed $D \bar{D}, D \bar{D}^{*}$ and $D^{*} \bar{D}^{*}$ decay modes 106$]$. However, a mass of less than $4000 \mathrm{MeV}$ is in conflict with quenched lattice computations of the low lying hybrid spectrum[54]. Thus more data are required before the exotic nature of the $Y$ can be claimed with any certainty.
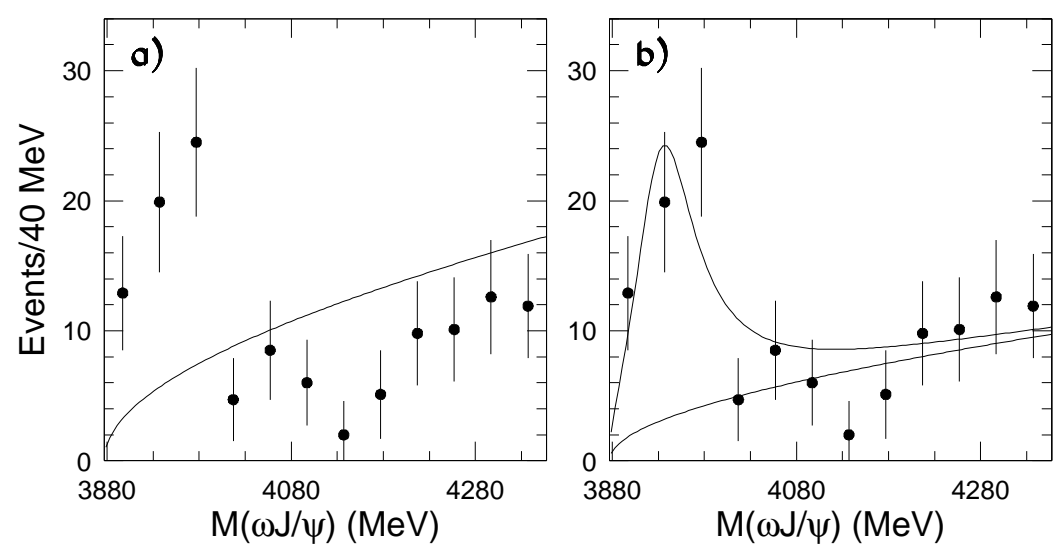

FIG. 23: Threshold Enhancement in the $\omega J / \psi$ System (left) and the Breit Wigner Fit (right) 105].

E. $Z(3930)$

The $Z(3930)$ was observed by the Belle collaboration [107] in $\gamma \gamma \rightarrow D \bar{D}$ with a mass of $3931 \pm 4 \pm 2 \mathrm{MeV}$ and a width of $20 \pm 8 \pm 3 \mathrm{MeV}$ at a claimed significance of $5.5 \sigma$ (see Fig. 24). The $D \bar{D}$ helicity distribution was determined to be consistent with $J=2$. Due to its mass and decay mode, the $Z$ may be considered a $\chi(2 P)$ candidate along with the $X(3940)$ and $Y(3940)$. In this case identification as the $\chi_{c 2}^{\prime}$ is natural. 


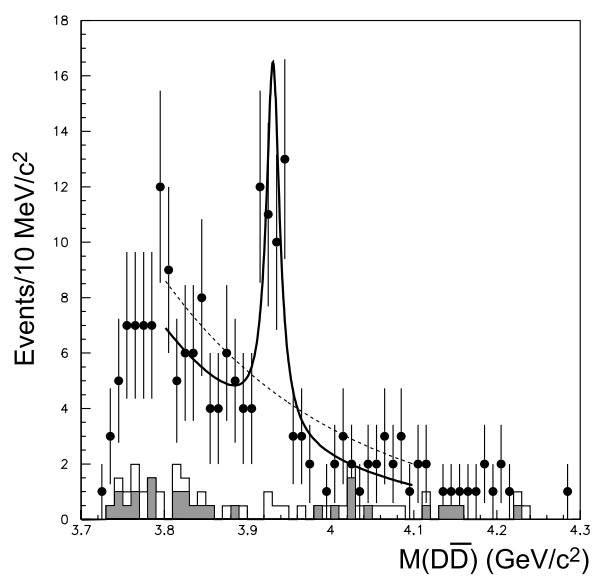

FIG. 24: Observation of the $Z(3930)$ in $\gamma \gamma \rightarrow D \bar{D}[107$.

The predicted mass of the $\chi_{c 2}^{\prime}$ is $3970-3980 \mathrm{MeV}$ (Table I) and the predicted width is $60 \mathrm{MeV}$ (Table IV). However, setting the mass to the measured $3931 \mathrm{MeV}$ restricts phase space sufficiently that the predicted strong width drops to $35 \mathrm{MeV}$, reasonably close to the measurement. The predicted branching fraction to $D \bar{D}$ is $70 \%$. Furthermore, the largest radiative transition is $\chi_{c 2}^{\prime} \rightarrow \psi^{\prime} \gamma$ with a rate of $180 \pm 30 \mathrm{keV}[13]$.

If this assignment is correct it implies a $40 \mathrm{MeV}$ error in the quark model predictions, somewhat large compared to the average errors shown in Table III and this is perhaps an indication that the expected breakdown in the model is occurring. Furthermore, if the $X(3940)$ is the $\chi_{c 1}^{\prime}$ then these excited $P$ waves exhibit an inverted spin splitting of $-10 \mathrm{MeV}$ rather than the expected $+45 \mathrm{MeV}$. The perturbative $\chi_{c 2}-\chi_{c 1}$ splitting is given by $\left\langle 16 \alpha_{s} /\left(5 r^{3}\right)-b / r\right\rangle / m^{2}$, so an inverted splitting is possible, but indicates a serious lack of understanding of the relevant dynamics. If this scenario is confirmed then the $\chi_{c 0}^{\prime}$ is expected at $M\left(\chi_{c 1}^{\prime}\right)-1 / 2\left\langle 8 \alpha_{s} / r^{3}-b / r\right\rangle / m^{2}$. This is difficult to estimate in view of the uncertainty in the dynamics. A reasonable guess may be a $\chi_{c 0}^{\prime}$ mass of $3945 \mathrm{MeV}$. Alternatively, if the mass scale has only been misunderstood, then a $\chi_{c 0}^{\prime}$ is expected at approximately $3810 \mathrm{MeV}$ - it is possible that a glimpse of this state is already visible in Fig. 24]

In view of the uncertainty in the masses of the $\chi_{c J}^{\prime}$ it is worth examining the partial widths of the $\chi_{c 0}^{\prime}$ and $\chi_{c 2}^{\prime}$ as a function of the resonance mass. The results are shown in Fig. 25] and indicate that the $\chi_{c 0}^{\prime}$ can be very narrow near $3920 \mathrm{MeV}$. In this case it may not be a surprise that this state is not strongly visible in Fig. 24.
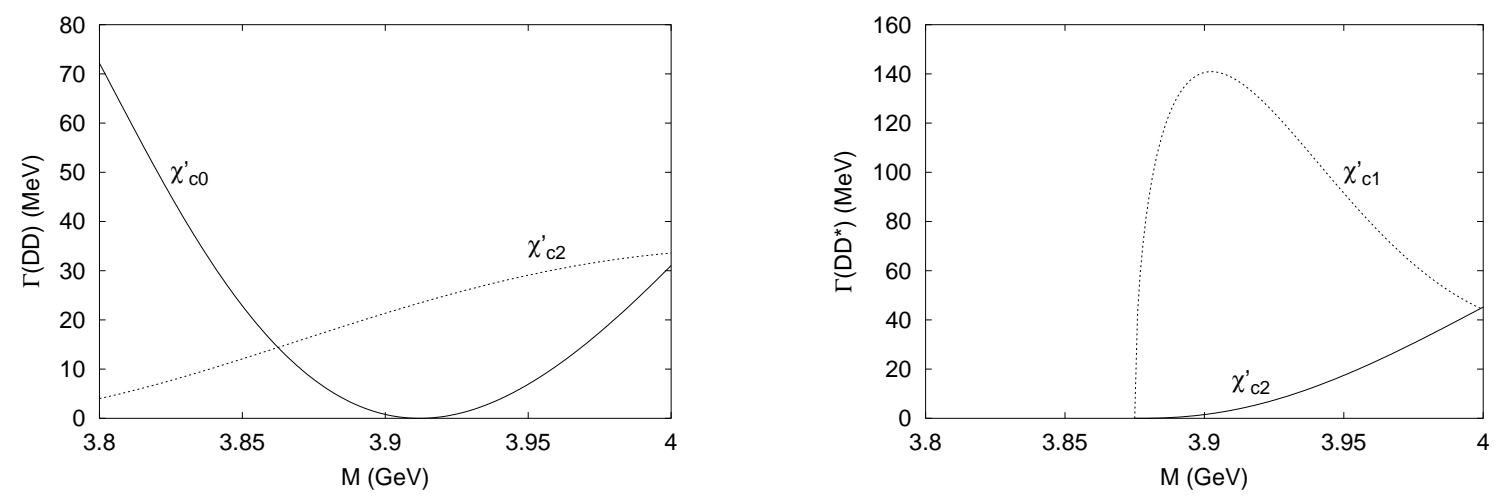

FIG. 25: $D \bar{D}$ and $D \bar{D}^{*}$ Partial Widths vs. $\chi_{c J}^{\prime}$ Mass.

The production rate in $\gamma \gamma$ has been determined to be

$$
\Gamma_{\gamma \gamma} \operatorname{Br}(Z \rightarrow D \bar{D})=0.23 \pm 0.06 \pm 0.04 \mathrm{keV}
$$

The ${ }^{3} P_{0}$ model results predict a $D \bar{D}$ branching ratio of $70 \%$ for the $\chi_{c 2}^{\prime}$ and hence $\Gamma(\gamma \gamma)=0.33 \mathrm{keV}$. This rate has been estimated by Barnes 108 to be $0.64 \mathrm{keV}$ - given the uncertainties, this is reasonably close to the data.

Searching for $D \bar{D}^{*}$ decay modes of the $Z$ will be an important diagnostic for this state. Measuring helicity angle 
distributions for the $X, Y$, and $Z$ will also be important for determining quantum numbers. Finally, increasing statistics for all these states will be useful.

F. $Y(4260)$

The $Y(4260)$ is an intriguing recent addition to the charmonium spectrum. It was discovered by BaBar 109 as an enhancement in the $\pi \pi J / \psi$ subsystem of the reaction $e^{+} e^{-} \rightarrow \gamma_{\mathrm{ISR}} J / \psi \pi \pi$ (see Fig. 26). The measured mass is $4259 \pm 8 \pm 4 \mathrm{MeV}$ and the width is $88 \pm 23 \pm 5 \mathrm{MeV}$; with a total of $125 \pm 23$ events in the signal. The $\pi \pi$ mass distribution peaks near $1 \mathrm{GeV}$ (see Fig. 27), suggesting an isobar $Y \rightarrow J / \psi f_{0}(980) \rightarrow J / \psi \pi \pi$ decay (although there is substantial strength at lower invariant mass suggesting a $\sigma$ contribution). The reaction rate has also been determined to be

$$
\Gamma\left(Y \rightarrow e^{+} e^{-}\right) \operatorname{Br}(Y \rightarrow J / \psi \pi \pi)=5.5 \pm 1.0_{-0.7}^{+0.8} \mathrm{eV}
$$

The $Y$ is not seen in $e^{+} e^{-} \rightarrow$ hadrons, in particular in the final states $D \bar{D}$ or $D_{s} \bar{D}_{s}$, indicating that the branching fraction of the $Y$ to $J / \psi \pi \pi$ is large compared to that for the $\psi(3770)$.

BaBar have also searched for the $Y$ in $B$ decays and report 110 ] "an excess of events" around $4.3 \mathrm{GeV}$ at a significance of $3 \sigma$. The measured branching ratio is

$$
\operatorname{Br}\left(B^{-} \rightarrow K^{-} Y\right) \operatorname{Br}(Y \rightarrow J / \psi \pi \pi)=(2.0 \pm 0.7 \pm 0.2) \cdot 10^{-5}
$$

If the $J / \psi \pi \pi$ mode dominates the width of the $Y$ this implies a $B$ decay branching ratio of approximately $10^{-5}$, two orders of magnitude smaller that the branching fractions for $K J / \psi$ or $K \psi^{\prime}$. Similarly, $\Gamma\left(Y \rightarrow e^{+} e^{-}\right) \sim 5$ eV, substantially smaller than typical vector leptonic widths.

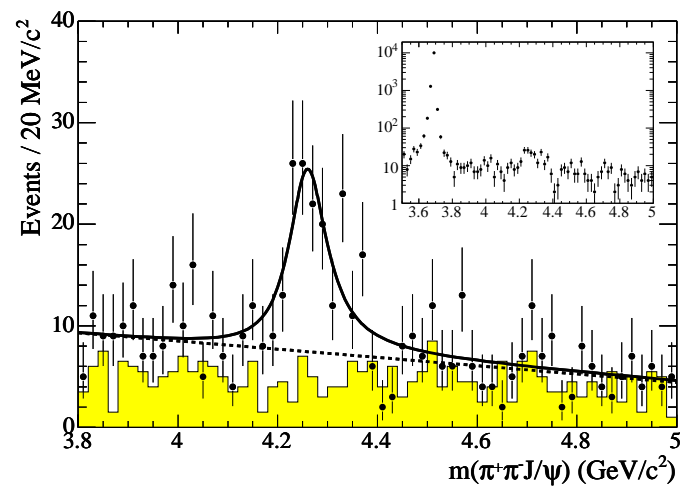

FIG. 26: The $Y(4260)$ Observed in $e^{+} e^{-} \rightarrow \gamma_{I S R} J / \psi \pi \pi[109]$.

Evidently the $Y$ is a vector with $c \bar{c}$ flavour content. Of course the low lying charmonium vectors are well known (see Table III) and the first mesonic charmonium vector available is the $3^{3} D_{1}$ state. However quark model estimates of its mass place it at $4460 \mathrm{MeV}$, much too heavy for the $Y$. Of course this statement relies on the robustness of the quark model. Thus, for example, Llanes-Estrada 111] has argued that the $Y$ is the $\psi(4 S)$ based on the spectrum of a relativistic model. This displaces the $\psi(4415)$ which moves to the $\psi(5 S)$ position.

A variety of other explanations for the $Y$ have been proposed. Among these are the suggestion that the $Y$ is a $\Lambda_{c} \bar{\Lambda}_{c}$ baryonium [112]. Maiani et al. 113] claim the $Y$ is the first orbital excitation of a tetraquark $[c s]_{S}[\bar{c} \bar{s}]_{S}$ state which decays predominantly to $D_{s} \bar{D}_{s}$. A nonet of vector $Y$ states is predicted with masses $Y(I=0)=3910 \mathrm{MeV}$ and $Y(I=1 / 2)=4100 \mathrm{MeV}$. Alternatively, Liu, Zeng, and Li suggest that the $Y$ is a $\chi_{c 1} \rho$ molecule bound by $\sigma$ exchange 114]. Thus two isotriplet partner states are predicted and possible isotriplet $\chi_{c 0} \rho$ and $\chi_{c 2} \rho$ states may exist as well.

Of the charmonium states which we know must exist, the most natural explanation is as a $c \bar{c}$ hybrid [115, 116, 117]. A hybrid assignment agrees with the large $J / \psi \pi \pi$ width $^{11}$, small leptonic coupling, and small $B$ decay branching

11 Although it remains to be established that a large $J / \psi \pi \pi$ mode is inconsistent with canonical charmonium. 


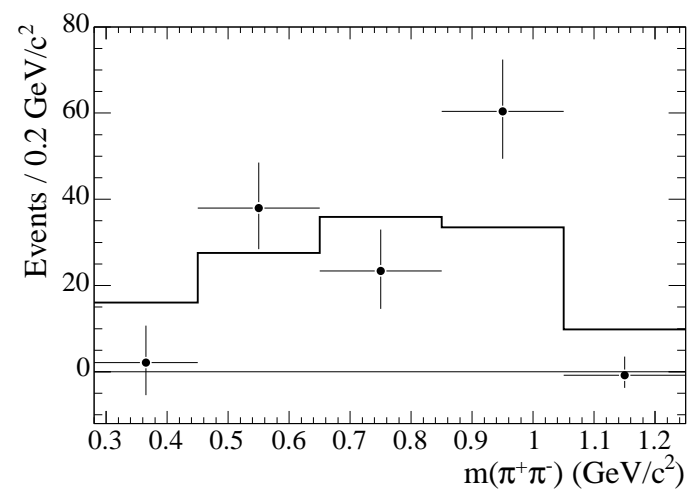

FIG. 27: $\pi \pi$ Invariant Mass Distribution [109]. The line corresponds to an S-wave phase space model.

fraction. As stated in the Introduction, the lightest charmonium hybrid is expected at $4400 \mathrm{MeV}-$ somewhat high, but perhaps acceptable given our lack of experience in this sector. The observed decay to a closed charm final state is simply explained: all hybrid decay models forbid decays to identical mesons, and many forbid decays to pairs of S-wave mesons 106]. Thus the allowed channels for a vector hybrid are $D \bar{D}^{*}$ (which is suppressed), $D_{2} \bar{D}$ (a suppressed $D$-wave mode), $D_{1} \bar{D}$, and $D_{1}^{\prime} \bar{D}$. The latter modes are above threshold and thus are very small. Ref. [117 reminds us of these facts in the context of a constituent glue model ${ }^{12}$. Thus the decays of a putative vector hybrid at $4260 \mathrm{MeV}$ will be dominated by closed charm channels. The UKQCD collaboration 119] has found a large coupling of $b \bar{b}$ hybrids to closed flavour modes such as $\chi_{b} \sigma$. Such a large coupling will be required to accommodate the relatively large total width of the $Y$. Understanding the mechanisms behind this coupling will then be a major task for phenomenologists.

If the $Y$ is indeed a vector $c \bar{c}$ hybrid, it should also decay to $J / \psi \sigma, J / \psi K \bar{K}, \chi_{c 0} \omega, h_{c} \eta$, and $h_{c} \eta^{\prime}$ and these modes should be sought. Furthermore, one may expect nearby pseudoscalar and exotic $J^{P C}=1^{-+}$hybrids which will display their own unique decay patterns [116].

The proximity of the $Y$ to $D \bar{D}_{1}$ threshold (at $4285 \mathrm{MeV}$ ) suggests that the resonance could actually be a cusp at $D \bar{D}_{1}$ threshold combined with rescattering into the $J / \psi f_{0} \rightarrow J / \psi \pi \pi$ channel. Confirming the phase motion of the $Y$ is thus an important (although very difficult) task. Searching for a $D \bar{D} \pi \pi$ decay mode with the $D \pi \pi$ subsystem dominated by a virtual $D_{1}$ would also be an important diagnostic. Furthermore, if rescattering drives the $Y$ enhancement then one may expect similar enhancements at $D \bar{D}_{s 1}$ (and similar) thresholds. These may possibly be seen in the $J / \psi K \bar{K}$ channel.

In line with the previous discussion, it is tempting to speculate on molecular interpretations for this state. In particular the $D D_{1}$ channel is an S-wave threshold and is thus a likely one for forming a molecule. However, if the system does bind, it does so with a novel mechanism since pion exchange does not lead to a diagonal interaction in this channel (unlike the case of the $X(3872)$ ). It is possible that off-diagonal interactions may provide the required novelty. For example, $D \bar{D}_{1} \rightarrow D^{*} \bar{D}^{*} \rightarrow D \bar{D}_{1}$ via iterated pion exchange; however, it seems unlikely that such a mechanism can lead to binding. Countering this, is a recent lattice computation that claims to see a four-quark state at $4238 \pm 31 \mathrm{MeV}$ and predicts a $c \bar{c} s \bar{s}$ at $4450 \pm 100 \mathrm{MeV}[120]$.

Lastly, the reader may be disturbed that the $Y$ appears in ISR but is not seen $e^{+} e^{-}$annihilation. This issue has been considered in Ref. [109] where the $Y$ cross section in $e^{+} e^{-}$was estimated to be $50 \mathrm{pb}$. This corresponds to $0.34 \%$ of the signal at $\sqrt{s}=4.25 \mathrm{GeV}$, which is not experimentally discernible. Thus the ISR production mechanism serves as a useful filter for some vector channels, such as $\pi \pi J / \psi$.

12 Detailed predictions of constituent glue models of hybrids should be regarded with care since they do not agree with level orderings of adiabatic hybrid surfaces as computed on the lattice unless specific gluodynamics are assumed [118]. 


\section{NEW OPEN CHARM STATES}

The new revolution in charmonia was contemporaneous with BaBar's discovery of an enigmatic open charm state, the $D_{s}(2317)$ [121]. We shall proceed with a review of theoretical expectations for heavy-light mesons before moving on to the properties and interpretations of the new open charm states.

\section{A. Heavy Quark Symmetry and the Quark Model}

\section{Notation}

Generic $Q \bar{q}$ states will be denoted as $D$ in the following. Properties of the six lowest lying $D$ s are listed in Table XIII Note that charge conjugation is no longer a symmetry in the heavy-light sector; thus the ${ }^{3} P_{1}$ and ${ }^{1} P_{1}$ states mix to form the physical $D_{1}$ and $D_{1}^{\prime}$. Quark model assignments for the remaining states are as in the quarkonium sector.

In the $m_{Q} \rightarrow \infty$ limit the heavy quark acts as a static colour source and it is useful to describe the system in the $j j$ coupling scheme. Thus the light quark degrees of freedom are written as $j_{q}=\ell_{q}+S_{q}$ where $\ell_{q}\left(S_{q}\right)$ is the angular momentum (spin) of the light quark. The total spin-parity is then given by $J^{P}=\frac{1}{2}^{-} \otimes j_{q}^{p}$.

The heavy-light system can be very different from quarkonium. For example, quarkonia contain a hierarchy of scales, $m_{Q},\langle p\rangle=\alpha_{s} m_{Q}, E=\alpha_{s}^{2} m_{Q}$, etc, whereas a heavy-light system has two scales, $m_{Q}$ and $\Lambda_{Q C D}$. This can have profound implications, for example, hyperfine splitting for heavy quarkonium must scale as $\delta M_{\text {hyp }} \sim m_{Q}$ whereas it scales as $\delta M_{h y p} \sim \Lambda_{Q C D}^{2} / m_{Q}$ for heavy-light systems.

TABLE XIII: Low-Lying $Q \bar{q}$ States

\begin{tabular}{|c|c|c|c|c|c|}
\hline property & $\overline{D^{*}}$ & $\overline{D_{0}}$ & $D_{1}$ & $D_{1}^{\prime}$ & $\overline{D_{2}}$ \\
\hline $\begin{array}{l}J^{P} \\
(2 S+1) L_{J}\end{array}$ & $\begin{array}{|cc|}0^{-} & 1^{-} \\
{ }^{1} S_{0} & { }^{3} S_{1}-{ }^{3} D_{1}\end{array}$ & $\begin{array}{l}0^{+} \\
{ }^{3} P_{0}\end{array}$ & $\begin{array}{c}1^{+} \\
{ }^{3} P_{1}-{ }^{1} P_{1}\end{array}$ & $\begin{array}{c}1^{+} \\
{ }^{3} P_{1}-{ }^{1} P_{1}\end{array}$ & $\begin{array}{c}2^{+} \\
{ }^{3} P_{2}\end{array}$ \\
\hline$\ell_{q}$ & $0 \quad 0$ & 1 & 1 & 1 & 1 \\
\hline $\begin{array}{l}j_{q}^{p} \\
\text { decay }\end{array}$ & $\frac{1}{2}^{-} \quad \frac{1}{2}^{-}$ & $\begin{array}{c}\frac{1}{2}^{+} \\
(D P)_{S}\end{array}$ & $\begin{array}{c}\frac{1}{2}^{+} \\
\left(D^{*} P\right)_{S}\end{array}$ & $\begin{array}{c}\frac{3}{2}^{+} \\
\left(D^{*} P\right)_{D}\end{array}$ & $\begin{array}{c}\frac{3}{2}^{+} \\
(D P)_{D}\end{array}$ \\
\hline
\end{tabular}

\section{Spin Splitting and Multiplet Structure}

The different bases impose different multiplet structures on the $D$ spectrum. In the absence of spin splitting, the spectroscopic $L S$ basis has an S-wave $\left(D, D^{*}\right)$ doublet and a $\mathrm{P}$-wave quadruplet. Alternatively, the heavy quark basis contains three doublets labelled by $j_{q}^{p}=\frac{1}{2}^{-}, \frac{1}{2}^{+}$, and $\frac{3}{2}^{+}$.

The spin splittings induced in the LS basis may be determined by evaluating matrix elements of the interactions of Eq. 2 In the following only terms of order $1 / m_{q}^{2}$ or $1 / m_{q} m_{Q}$ are retained. The perturbative hyperfine splittings is

$$
\begin{aligned}
\delta M(D) & =-\frac{3}{4} \frac{\left\langle V_{4}\right\rangle}{3 m_{Q} m_{q}} \\
\delta M\left(D^{*}\right) & =+\frac{1}{4} \frac{\left\langle V_{4}\right\rangle}{3 m_{Q} m_{q}}
\end{aligned}
$$

The P-waves are split by tensor and spin-orbit interactions according to

$$
\begin{aligned}
& \delta M\left(D_{0}\right)=-\frac{\left\langle V_{S O}\right\rangle}{m_{q}^{2}} \\
& \delta M\left(D_{2}\right)=\frac{1}{2} \frac{\left\langle V_{S O}\right\rangle}{m_{q}^{2}}
\end{aligned}
$$




$$
\delta M\left({ }^{3} P_{1},{ }^{1} P_{1}\right)=-\frac{1}{2} \frac{\left\langle V_{S O}\right\rangle}{m_{q}^{2}}\left(\begin{array}{cc}
1 & -\sqrt{2} \\
-\sqrt{2} & 0
\end{array}\right)
$$

Here $V_{S O}$ is given by

$$
V_{S O}=\frac{\epsilon^{\prime}}{2 r}+\frac{V_{1}^{\prime}}{r}+\frac{1}{2} V_{5}\left(m_{q}, m_{Q}\right) .
$$

The sign of the off-diagonal term in the P-wave splitting is convention dependent. Here I have chosen to work with $q \bar{Q}$ mesons and to combine angular momentum and spin in the order $L \times S$.

Tensor splittings are necessarily of order $1 / m_{Q} m_{q}$ and are given by

$$
\begin{aligned}
& \delta M\left(D_{0}\right)=-4 \frac{\left\langle V_{3}\right\rangle}{12 m_{Q} m_{q}} \\
& \delta M\left(D_{2}\right)=-\frac{2}{5} \frac{\left\langle V_{3}\right\rangle}{12 m_{Q} m_{q}} \\
& \delta M\left({ }^{3} P_{1}\right)=+2 \frac{\left\langle V_{3}\right\rangle}{12 m_{Q} m_{q}}
\end{aligned}
$$

Diagonalising the spin-orbit splittings in the heavy quark limit yields

$$
M\left(D_{2}\right)=M\left(D_{1}^{\prime}\right)=M_{0}+\frac{\left\langle V_{S O}\right\rangle}{2 m_{q}^{2}}
$$

and

$$
M\left(D_{0}\right)=M\left(D_{1}\right)=M_{0}-\frac{\left\langle V_{S O}\right\rangle}{m_{q}^{2}}
$$

in agreement with the $j j$ multiplets. The physical $D_{1}$ and $D_{1}^{\prime}$ states are defined in terms of a mixing angle:

$$
\begin{aligned}
\left|D_{1}\right\rangle & =+\cos (\phi)\left|{ }^{1} \mathrm{P}_{1}\right\rangle+\sin (\phi)\left|{ }^{3} \mathrm{P}_{1}\right\rangle \\
\left|D_{1}^{\prime}\right\rangle & =-\sin (\phi)\left|{ }^{1} \mathrm{P}_{1}\right\rangle+\cos (\phi)\left|{ }^{3} \mathrm{P}_{1}\right\rangle .
\end{aligned}
$$

In the heavy-quark limit a particular mixing angle follows from the quark mass dependence of the spin-orbit and tensor terms, which is $\phi_{H Q}=-54.7^{\circ}\left(35.3^{\circ}\right)$ if the expectation of the heavy-quark spin-orbit interaction is positive (negative) [122]. Since the former implies that the $2^{+}$state is greater in mass than the $0^{+}$state, and this agrees with experiment, we employ $\phi=-54.7^{\circ}$ in the following. This implies

$$
\begin{aligned}
& \left.\left.\left|D_{1}\right\rangle_{H Q}=+\left.\frac{1}{\sqrt{3}}\right|^{1} P_{1}\right\rangle-\left.\sqrt{\frac{2}{3}}\right|^{3} P_{1}\right\rangle \\
& \left.\left|D_{1}^{\prime}\right\rangle_{H Q}=+\sqrt{\frac{2}{3}}\left|{ }^{1} P_{1}\right\rangle+\left.\frac{1}{\sqrt{3}}\right|^{3} P_{1}\right\rangle .
\end{aligned}
$$

\section{Decays}

The $\mathrm{P}$-wave $D$ mesons are sufficiently light that the only isospin conserving decays are to $D P$ or $D^{*} P$ where $P$ is a pseudoscalar meson ( $\pi$ for $D$ decays and $K$ for $D_{s}$ decays). Conservation of angular momentum implies that the 
$D_{0}$ can only couple to $D P$ in an S-wave and one may thus expect the $D_{0}$ to be broad. Similarly the $D_{2}$ only couples to $D P$ in a $\mathrm{P}$-wave and is narrow.

In general a $D_{1}$ or $D_{1}^{\prime}$ may decay to $D^{*} P$ in $\mathrm{S}$ - and D-wave. However, the conservation of heavy quark spin implies that the $D_{1}$ only decays in an S-wave while the $D_{1}^{\prime}$ decays in a D-wave. Thus the $j_{q}=\frac{1}{2}$ doublet is broad and the $j_{q}=\frac{3}{2}$ doublet is narrow. Fig. [28 illustrates this selection rule 122, 123]. We consider an initial $D_{1}^{(\prime)}$ decaying into a $D^{*}$ and a light pseudoscalar meson. Conservation of angular momentum and heavy quark spin implies that

$$
j_{q}\left(D_{1}^{(\prime)}\right)=j_{q}\left(D^{*}\right)+L_{D^{*} P}
$$

Setting $j_{q}\left(D^{*}\right)=\frac{1}{2}$ and $j_{q}\left(D_{1}\right)=\frac{1}{2}$ then implies that the relative angular momentum in the final state is $L_{D^{*} P}=0$. Alternatively for the $D_{1}^{\prime}$ one has $j_{q}\left(D_{1}^{\prime}\right)=\frac{3}{2}$ and hence $L_{D^{*} P}=2$.

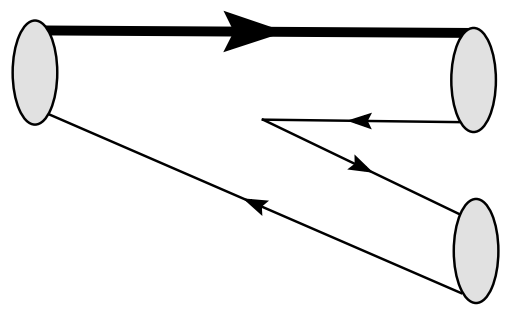

FIG. 28: P-wave Decay in the Heavy Quark Limit.

It is interesting to examine this behaviour in the ${ }^{3} P_{0}$ model. One finds the following relation between ${ }^{3} P_{1}$ and ${ }^{1} P_{1}$ decay amplitudes 26,122

$$
\begin{aligned}
& \mathcal{A}\left({ }^{1} P_{1} \rightarrow V P\right)_{S}=-\frac{1}{\sqrt{2}} \mathcal{A}\left({ }^{3} P_{1} \rightarrow V P\right)_{S} \\
& \mathcal{A}\left({ }^{1} P_{1} \rightarrow V P\right)_{D}=\sqrt{2} \mathcal{A}\left({ }^{3} P_{1} \rightarrow V P\right)_{D}
\end{aligned}
$$

Eq. 105 then implies

$$
\begin{aligned}
\mathcal{A}\left(D_{1} \rightarrow V P\right)_{S} & =\left(-\frac{\cos \phi}{\sqrt{2}}+\sin \phi\right) \mathcal{A}_{S} \\
\mathcal{A}\left(D_{1} \rightarrow V P\right)_{D} & =(\sqrt{2} \cos \phi+\sin \phi) \mathcal{A}_{D} \\
\mathcal{A}\left(D_{1}^{\prime} \rightarrow V P\right)_{S} & =\left(\frac{\sin \phi}{\sqrt{2}}+\cos \phi\right) \mathcal{A}_{S} \\
\mathcal{A}\left(D_{1}^{\prime} \rightarrow V P\right)_{D} & =(-\sqrt{2} \sin \phi+\cos \phi) \mathcal{A}_{D}
\end{aligned}
$$

Substituting the heavy quark mixing angle $\cos \phi=1 / \sqrt{3}$ yields

$$
\begin{aligned}
\mathcal{A}\left(D_{1} \rightarrow V P\right)_{S} & =-\sqrt{\frac{3}{2}} \mathcal{A}_{S} \\
\mathcal{A}\left(D_{1} \rightarrow V P\right)_{D} & =0 \\
\mathcal{A}\left(D_{1}^{\prime} \rightarrow V P\right)_{S} & =0 \\
\mathcal{A}\left(D_{1}^{\prime} \rightarrow V P\right)_{D} & =\sqrt{3} \mathcal{A}_{D}
\end{aligned}
$$

in agreement with the heavy quark selection rule. The agreement is likely quite general because relations 108 hold in the Cornell model as well. This may be true because the nonrelativistic structure of the ${ }^{3} P_{0}$ and Cornell transition operators are very similar:

$$
H_{3 P 0} \sim \sum_{k} b_{k}^{\dagger} \sigma \cdot k d_{-k}^{\dagger}
$$


while

$$
H_{\text {Cornell }} \sim \mathbb{I} \cdot V(q) \sum_{k} b_{k+q}^{\dagger} \sigma \cdot q d_{-k}^{\dagger} .
$$

Additional selection rules may be derived $[26]$. For example the following relations hold in the ${ }^{3} P_{0}$ model

$$
\begin{aligned}
\mathcal{A}\left(2^{3} S_{1} \rightarrow{ }^{1} P_{1} P\right)_{S} & =-\frac{1}{\sqrt{2}} \mathcal{A}\left(2^{3} S_{1} \rightarrow{ }^{3} P_{1} P\right)_{S} \\
\mathcal{A}\left(2^{3} S_{1} \rightarrow{ }^{1} P_{1} P\right)_{D} & =\sqrt{2} \mathcal{A}\left(2^{3} S_{1} \rightarrow{ }^{3} P_{1} P\right)_{D} \\
\mathcal{A}\left({ }^{3} D_{1} \rightarrow{ }^{1} P_{1} P\right)_{S} & =\sqrt{2} \mathcal{A}\left({ }^{3} D_{1} \rightarrow{ }^{3} P_{1} P\right)_{S}
\end{aligned}
$$

Thus the $D_{1} \pi\left(D_{1}^{\prime} \pi\right)$ mode will be large (small) in $D\left(2^{3} S_{1}\right)$ decays and the $D_{1} \pi\left(D_{1}^{\prime} \pi\right)$ mode will be small (large) in $D\left({ }^{3} D_{1}\right)$ decays.

\section{Spin Splitting with Continuum Mixing}

Although the conclusions reached above seem to be in accord with data (see Sect. IV C), the logic used - namely first perform perturbation theory in the spin orbit interaction, then use the perturbed states to compute decay widths - is not correct. In general the coupling to continuum channels also perturbs the energy and structure of the $D_{1}$ s; hence spin orbit and loop effects should be considered at the same time. In this case the perturbative mass splittings are given by

$$
\begin{aligned}
& \delta M\left(D_{0}\right)=-\frac{\left\langle V_{S O}\right\rangle}{m_{q}^{2}}+\int \frac{d^{3} k}{(2 \pi)^{3}} \frac{\left|\omega_{0}(k)\right|^{2}}{M_{0}-E_{D P}(k)} \\
& \delta M\left(D_{2}\right)=+\frac{\left\langle V_{S O}\right\rangle}{2 m_{q}^{2}}+\int \frac{d^{3} k}{(2 \pi)^{3}} \frac{\left|\omega_{2}(k)\right|^{2}}{M_{0}-E_{D P}(k)}
\end{aligned}
$$

where $\omega_{J}(k)=\left\langle D_{J}|V| D P ; \vec{k}\right\rangle$ and $V$ is, for example, the ${ }^{3} P_{0}$ transition operator.

The $D_{1}$ and $D_{1}^{\prime}$ eigenstates are determined by diagonalising a Hamiltonian describing the interactions of the ${ }^{1} P_{1}$, ${ }^{3} P_{1},(D P)_{S}$ and $(D P)_{D}$ channels. Integrating out the continuum channels and examining the perturbative mass shifts yields an effective $2 \times 2$ Hamiltonian to be solved for the physical $D_{1}$ states:

$\delta H=-\frac{1}{2} \frac{\left\langle V_{S O}\right\rangle}{m_{q}^{2}}\left(\begin{array}{cc}1 & -\sqrt{2} \\ -\sqrt{2} & 0\end{array}\right)+\int \frac{d^{3} k}{(2 \pi)^{3}} \frac{1}{M_{0}-E_{D^{*} P}}\left(\begin{array}{cc}\left(2\left|\tilde{\omega}_{0}(k)\right|^{2}+\frac{1}{2}\left|\tilde{\omega}_{2}(k)\right|^{2}\right) & \left(-\sqrt{2}\left|\tilde{\omega}_{0}(k)\right|^{2}+\frac{1}{\sqrt{2}}\left|\tilde{\omega}_{2}(k)\right|^{2}\right) \\ \left(-\sqrt{2}\left|\tilde{\omega}_{0}(k)\right|^{2}+\frac{1}{\sqrt{2}}\left|\tilde{\omega}_{2}(k)\right|^{2}\right) & \left(\left|\tilde{\omega}_{0}(k)\right|^{2}+\left|\tilde{\omega}_{2}(k)\right|^{2}\right)\end{array}\right)$.

where $\tilde{\omega}_{L}(k)=\left\langle{ }^{1} P_{1}|V| D^{*} P ; k\right\rangle_{L}$. The continuum contribution to $\delta H$ may be written as

$$
\delta H_{\text {cont }}=\int \frac{d^{3} k}{(2 \pi)^{3}} \frac{\left|\tilde{\omega}_{0}(k)\right|^{2}+\left|\tilde{\omega}_{2}(k)\right|^{2}}{M_{0}-E_{D^{*} P}}\left(\begin{array}{cc}
1 & 0 \\
0 & 1
\end{array}\right)+\int \frac{d^{3} k}{(2 \pi)^{3}} \frac{\left|\tilde{\omega}_{0}(k)\right|^{2}-\frac{1}{2}\left|\tilde{\omega}_{2}(k)\right|^{2}}{M_{0}-E_{D^{*} P}}\left(\begin{array}{cc}
1 & -\sqrt{2} \\
-\sqrt{2} & 0
\end{array}\right)
$$

showing that the eigenvalues and mixing angle remain unchanged from the naive mixing case.

The full perturbative mass shifts are

$$
\delta M\left(D_{1}\right)=-\frac{\left\langle V_{S O}\right\rangle}{m_{q}^{2}}+3 \int \frac{d^{3} k}{(2 \pi)^{3}} \frac{\left|\tilde{\omega}_{0}(k)\right|^{2}}{M_{0}-E_{D^{*} P}}
$$

and

$$
\delta M\left(D_{1}^{\prime}\right)=\frac{1}{2} \frac{\left\langle V_{S O}\right\rangle}{m_{q}^{2}}+\frac{3}{2} \int \frac{d^{3} k}{(2 \pi)^{3}} \frac{\left|\tilde{\omega}_{2}(k)\right|^{2}}{M_{0}-E_{D^{*} P}}
$$


Finally, in the heavy quark limit $E_{D P}=E_{D^{*} P}$ and $\omega_{0}=-\sqrt{3} \tilde{\omega}_{0}$. Thus the $\left(D_{0}, D_{1}\right)$ doublet is recovered. However, $\omega_{2}=-\sqrt{3 / 5} \tilde{\omega}_{2}$ and the heavy doublet $\left(D_{1}^{\prime}, D_{2}\right)$ is not recovered. This is not a great surprise - while it was established that the heavy doublet was narrow, there is no reason to believe that the widths of the $D_{1}^{\prime}$ and the $D_{2}$ are identical.

Furthermore, it should be recalled that all possible virtual continua contribute to the mass splitting. These continua do not necessarily obey the amplitude relations given in Eq. 108 and the naive heavy quark mixing angle and width relations need not hold. Also, the full computation should not be made in perturbation theory because some of the widths, and hence mass shifts, can be very large. In this case the concept of the mixing angle is not valid because the continuum cannot be represented as an effective potential but must be included in the Fock space expansion of the state

$$
\left|D_{1}\right\rangle=Z_{1}^{1 / 2}\left|{ }^{1} P_{1}\right\rangle+Z_{2}^{1 / 2}\left|{ }^{3} P_{1}\right\rangle+\int d^{3} k Z(k)^{1 / 2}\left|D^{*} P ; k\right\rangle .
$$

Alternatively, the Hilbert space becomes infinite dimensional rather than simply two dimensional.

\section{D-waves}

Mixing in the ${ }^{3} D_{2}-{ }^{1} D_{2}$ system may be addressed in the heavy quark limit of the constituent quark model as above. The mixing angle may be defined as

$$
\begin{aligned}
\left|D_{2}^{*}\right\rangle & \left.\left.=+\left.\cos \left(\phi_{D}\right)\right|^{1} D_{2}\right\rangle+\left.\sin \left(\phi_{D}\right)\right|^{3} D_{2}\right\rangle \\
\left|D_{2}^{* \prime}\right\rangle & \left.\left.=-\left.\sin \left(\phi_{D}\right)\right|^{1} D_{2}\right\rangle+\left.\cos \left(\phi_{D}\right)\right|^{3} D_{2}\right\rangle
\end{aligned}
$$

Proceeding as with $\mathrm{P}$-waves one finds a ${ }^{3} D_{2}-{ }^{1} D_{2}$ mixing matrix of

$$
M\left({ }^{3} D_{2},{ }^{1} D_{2}\right)=M_{0}-\frac{\left\langle V_{S O}\right\rangle}{2}\left(\begin{array}{cc}
1 & \sqrt{6} \\
\sqrt{6} & 0
\end{array}\right) .
$$

Diagonalising the spin-orbit interaction yields the results

$$
\begin{aligned}
& M\left({ }^{3} D_{1}\right)=M\left(D_{2}^{*}\right)=M_{0}-\frac{3}{2}\left\langle H_{S O}^{q}\right\rangle_{D} \\
& M\left({ }^{3} D_{3}\right)=M\left(D_{2}^{* \prime}\right)=M_{0}+\left\langle H_{S O}^{q}\right\rangle_{D},
\end{aligned}
$$

and a ${ }^{3} D_{2}-{ }^{1} D_{2}$ mixing angle of $\phi_{D}=-50.76^{\circ}$. Thus, the heavy quark $D$-wave states are

$$
\begin{aligned}
\left|D_{2}^{*}\right\rangle_{H Q} & \left.\left.=\left.\sqrt{\frac{2}{5}}\right|^{1} D_{2}\right\rangle-\left.\sqrt{\frac{3}{5}}\right|^{3} D_{2}\right\rangle \\
\left|D_{2}^{* \prime}\right\rangle_{H Q} & \left.=\sqrt{\frac{3}{5}}\left|{ }^{1} D_{2}\right\rangle+\left.\sqrt{\frac{2}{5}}\right|^{3} D_{2}\right\rangle .
\end{aligned}
$$

As with $P$-waves, the ${ }^{3} P_{0}$ strong decay model makes specific predictions for $D$-wave heavy quark decay amplitudes which may be useful in interpreting the spectroscopy. Some of these are:

$$
\begin{aligned}
& \mathcal{A}\left({ }^{1} D_{2} \rightarrow V P\right)_{P}=-\sqrt{\frac{2}{3}} \mathcal{A}\left({ }^{3} D_{2} \rightarrow V P\right)_{P} \\
& \mathcal{A}\left({ }^{1} D_{2} \rightarrow V P\right)_{F}=+\sqrt{\frac{3}{2}} \mathcal{A}\left({ }^{3} D_{2} \rightarrow V P\right)_{F} \\
& \mathcal{A}\left({ }^{1} D_{2} \rightarrow{ }^{1} P_{1} P\right)=0
\end{aligned}
$$

The second of these is an example of the ${ }^{3} P_{0}$ selection rule forbidding such transitions among $q \bar{q}$ spin singlets.

We note that, in analogy with the $P$-waves, the amplitude ratios above imply that the $D_{2}^{*}$ decays strongly in $P$ wave while the $D_{2}^{* \prime}$ decays only in $F$ wave and is thus narrower than the $D_{2}^{*}$. As with $P$-waves, this conclusion agrees with spin conservation in the heavy quark limit. Unfortunately, the ability to distinguish the states is weakened by the many other decay modes which exist for these states. Nevertheless, the $D_{2}^{*}$ and $D_{2}^{* \prime}$ have total widths which depend strongly on $\phi_{D}$ and measurement of any (or several) of the larger decay modes will provide (over) constrained tests of the model and measurements of the mixing angle. 


\section{B. Chiral Symmetry Breaking and Restoration in Heavy-Light Mesons}

\section{Effective Field Theory}

Ten years prior to the discovery of the $D_{s}(2317)$, Nowak et al. 124 and Bardeen and Hill 125 investigated the consequences of an effective lagrangian description of heavy-light mesons, called the chiral doublet model. Nowak et al. assumed heavy quark symmetry and broken chiral symmetry and thus arrived at the lagrangian of Eq. 65. Alternatively, Bardeen and Hill assumed that chiral symmetry was manifest and thus wrote an analogous lagrangian with the linear realisation of chiral symmetry. The properties of heavy-light mesons have also been examined in the context of a Nambu-Jona-Lasino model with chiral and heavy quark symmetries by Ebert et al. [126].

The combination of heavy quark and chiral symmetries imply that the bare low-lying heavy-light states are degenerate multiplets, $H$ and $G$ (or $H^{\prime}$ ) where

$$
H=\left(0^{-}, 1^{-}\right)
$$

and

$$
G=\left(0^{+}, 1^{+}\right)
$$

Note that this axial is the $j_{q}^{p}=\frac{1}{2}^{+}$state. Heavy quark symmetry imposes the doublet structure shown, while chiral symmetry implies that they are degenerate. This structure is modified in two ways: the heavy quark symmetry is explicitly broken by the finite heavy quark mass and chiral symmetry is spontaneously broken by nonperturbative vacuum gluodynamics (explicit chiral symmetry breaking is neglected). The subsequent mass splittings raise the $G$ multiplet above the $H$ by roughly the chiral symmetry breaking scale and induce identical hyperfine splittings in the $G$ and $H$ multiplets. Thus one has the prediction

$$
M\left(D_{1}\right)-M\left(D^{*}\right)=M\left(D_{0}\right)-M(D) \approx \Lambda_{Q C D}
$$

Both sets of authors have subsequently applied the formalism to the newly discovered $D_{s}$ states 127 . As we shall see, Eq. 138 is quite accurate:

$$
M\left(D_{s 1}\right)-M\left(D_{s}^{*}\right)=347.2 \pm 1.5 \mathrm{MeV}, \quad M\left(D_{s 0}\right)-M(D)=349.1 \pm 1.0 \mathrm{MeV}
$$

Additional predictions are [127]

$$
M\left(D_{0}\right)=2215 \mathrm{MeV}
$$

and

$$
M\left(D_{1}\right)=2357 \mathrm{MeV} .
$$

Experimentally these are $2352 \pm 50 \mathrm{MeV}$ and $2421.8 \pm 1.3 \mathrm{MeV}$ respectively $[1]$.

In the $B$ system the predictions are 127 .

$$
M\left(B_{0}\right)=5627 \pm 35 \mathrm{MeV}
$$

and

$$
M\left(B_{1}\right)=5674 \pm 35 \mathrm{MeV} .
$$

The PDG reports several narrow and broad resonances of undetermined quantum numbers at $5698 \mathrm{MeV}$, in rough agreement with these predictions.

The chiral nature of the lagrangian also permits computations of strong transitions involving Goldstone bosons. Indeed, parity doubling implies that the transition strength governing $\left(0^{+}, 1^{+}\right) \rightarrow\left(0^{-}, 1^{-}\right) \pi$ is related to the scalarpseudoscalar mass difference by a Goldberger-Treiman relationship 


$$
g_{\pi} f_{\pi}=M\left(0^{+}\right)-M\left(0^{-}\right)
$$

where $f_{\pi}$ is the pion decay constant. Unfortunately, these decays are kinematically forbidden for the $D_{s}$ system and they can only proceed via isospin breaking $D_{s} \rightarrow D \eta \rightarrow D \pi^{0}$ transitions. Similarly couplings to multiple pions may be computed; for example Bardeen et al.[127] predict

$$
\Gamma\left(D_{s 1} \rightarrow D_{s} \pi \pi\right)=4.2 \mathrm{keV} .
$$

It is worth stressing that the relationship shown in Eq. 138 is very difficult to obtain in the constituent quark model. In particular, the $D_{0}$ and $D_{1}$ are P-wave excitations with small hyperfine splittings. Thus the two sides of the equation are not related in any simple way. This issue will be examined in detail in the following section.

\section{An Explicit Chiral Doublet Model}

The speculation that chiral symmetry is relevant to heavy-light mesons is a vital part of the preceding arguments. While general arguments have been made that this should be true high in the meson or baryon spectra 128, it is not clear that chiral symmetry is relevant to low lying states. Thus it is useful to examine the issue of chiral symmetry restoration in the meson spectrum with the aid of a simple relativistic Nambu-Jona-Lasinio quark model. The model is defined by the Hamiltonian

$$
H=\int d^{3} x \psi^{\dagger}(-i \alpha \cdot \partial+\beta m) \psi+\frac{1}{2} \int d^{3} x d^{3} y \rho^{a}(x) V(x-y) \rho^{a}(y)
$$

where $\beta$ is the Dirac matrix $\gamma_{0}, \vec{\alpha}=\beta \vec{\gamma}$, and $\rho^{a}$ is the quark charge density current.

Chiral symmetry breaking occurs for a wide range of interactions, $V$ and is embodied in the gap equation [129]:

$$
Z_{\psi}(\Lambda) p s_{p}=[m(\Lambda)+\delta m(\Lambda)] c_{p}+\frac{C_{F}}{2} \int \frac{d^{3} k}{(2 \pi)^{3}} \tilde{V}(p, k)\left[s_{k} c_{p}-\hat{p} \cdot \hat{k} c_{k} s_{p}\right] .
$$

The functions $s_{k}$ and $c_{k}$ are defined in terms of the Bogoliubov angle $\phi(k)$ as $s_{k}=\sin \phi(k)$ and $c_{k}=\cos \phi(k)$. The constant $Z_{\psi}$ and the UV cutoff $\Lambda$ are required for renormalisation. However, this will not be discussed further since it is irrelevant to the following.

The quark gap equation is to be solved for the unknown Bogoliubov angle, which then specifies the quark vacuum and the quark field mode expansion via spinors of the form

$$
u_{s}(k)=\sqrt{\frac{1+s_{k}}{2}}\left(\begin{array}{c}
\chi_{s} \\
\frac{c_{k}}{1+s_{k}} \boldsymbol{\sigma} \cdot \hat{k} \chi_{s}
\end{array}\right) .
$$

Comparing the quark spinor to the canonical spinor (nonrelativistic normalisation is used) permits a simple interpretation of the Bogoliubov angle through the relationship $\mu(k)=k \tan \varphi(k)$ where $\mu$ may be interpreted as a dynamical momentum-dependent quark mass. Similarly $\mu(0)$ may be interpreted as a constituent quark mass.

Chiral and heavy-quark symmetry can be studied by projecting the Hamiltonian on pseudoscalar, scalar, vector, and axial vector $Q \bar{q}$ channels. For example, the pseudoscalar and scalar Tamm-Dancoff Salpeter equations are

$$
M_{0^{-}} \phi(k)=\left(E_{k}+\bar{E}_{k}\right) \phi(k)+\frac{1}{2} \int \frac{d^{3} p}{(2 \pi)^{3}}[a \hat{p} \cdot \hat{k}+b] \tilde{V}(p-k) \phi(p)
$$

and

$$
M_{0^{+}} \phi(k)=\left(E_{k}+\bar{E}_{k}\right) \phi(k)+\frac{1}{2} \int \frac{d^{3} p}{(2 \pi)^{3}}[b \hat{p} \cdot \hat{k}+a] \tilde{V}(p-k) \phi(p) .
$$

The factors in the interaction are given by 


$$
a=\sqrt{1-s_{q}} \sqrt{1-s_{k}} \sqrt{1+\bar{s}_{q}} \sqrt{1+\bar{s}_{k}}+\sqrt{1+s_{q}} \sqrt{1+s_{k}} \sqrt{1-\bar{s}_{q}} \sqrt{1-\bar{s}_{k}}
$$

and

$$
b=\sqrt{1+s_{q}} \sqrt{1+s_{k}} \sqrt{1+\bar{s}_{q}} \sqrt{1+\bar{s}_{k}}+\sqrt{1-s_{q}} \sqrt{1-s_{k}} \sqrt{1-\bar{s}_{q}} \sqrt{1-\bar{s}_{k}} .
$$

The function $s_{k}$ is related to the dynamical quark mass via $s_{k}=\mu(k) / \sqrt{k^{2}+\mu^{2}(k)}$.

The structure of these equations simplifies substantially in the nonrelativistic limit, $s_{k}, \bar{s}_{k} \rightarrow 1$. In this case the pseudoscalar interaction reduces to $2 \int V \phi$ while the scalar interaction reduces to $2 \int \hat{p} \cdot \hat{k} V \phi$. Thus the pseudoscalar interaction is S-wave, while the scalar interaction is $\mathrm{P}$-wave and one expects the scalar mass to be larger than the pseudoscalar. Of course these conclusions are in agreement with constituent quark models.

Alternatively, these equations reduce to

$$
M_{0^{ \pm}} \phi(k)=\left(E_{k}+\bar{E}_{k}\right) \phi(k)+\int \frac{d^{3} p}{(2 \pi)^{3}}[\hat{p} \cdot \hat{k}+1] \tilde{V}(p-k) \phi(p)
$$

in the heavy quark $\left(s_{k} \rightarrow 1\right)$ and chiral $\left(\bar{s}_{k} \rightarrow 0\right)$ limits. A similar results applies to the axial and vector equations. Thus the expected multiplet structure is recovered.

The effects of spontaneous chiral symmetry breaking may be examined by setting $\bar{s}_{k}=\mu(k) / \bar{E}(k)$. In this case the $\left(D_{0}, D_{1}\right)$ doublet is split from the $\left(D, D^{*}\right)$ doublet. Hyperfine interactions arising from a finite heavy quark mass then split the doublets. Bardeen and Hill assume that this splitting is identical in both doublets, leading to Eq. [138 This is consistent with the analysis here if the chiral splitting is small, or equivalently, $\left\langle s_{k}\right\rangle \ll 1$. However, experience with light mesons indicates that $\left\langle s_{k}\right\rangle \approx 1$ (or $\langle k\rangle<<\mu(0)$ ). If this is also true in heavy-light mesons (and we shall argue that it is) then the chirally split $D_{0}$ and $D$ are dominantly P- and S-wave respectively, and hence have very different hyperfine splittings and the conclusions of [124, 125, 127] are incorrect. In general one expects $\langle k\rangle \sim \mu(0) \sim \Lambda_{Q C D}$ so that $\left\langle s_{k}\right\rangle=\mathcal{O}(1)$. Alternatively, the function $\mu$ is universal (it is determined by the structure of the vacuum) and hence the ratio

$$
\frac{\left.\langle k\rangle\right|_{B^{*}}}{\left.\langle k\rangle\right|_{\rho}} \sim\left(\frac{\mu\left(B^{*}\right)}{\mu(\rho)}\right)^{1 / 3} \sim(2)^{1 / 3} \sim 1 .
$$

In summary, the scenario of Nowak et al. and Bardeen et al. relies on effective chiral symmetry restoration in low-lying heavy-light mesons but there is no reason to believe that this actually occurs. Of course this is a theoretical belief which flies in the face of the data (see the subsequent discussion); however, one must allow the possibility that other effects may exist which can explain the same data.

Finally, Jaffe et al. claim [130] that the notion of the irrelevance of chiral symmetry breaking in the spectrum cannot be correct under any circumstances and speculate that hints of parity restoration in the baryon spectrum are instead due to $U_{A}(1)$ restoration. These observations are at odds with those of Ref. [128] and the simple model presented here.

\section{C. $\quad D_{s}(2317)$ and $D_{s}(2460)$}

\section{Experiment}

BaBar's announcement 121] of a narrow low mass state seen in the $D_{s} \pi^{0}$ decay mode has generated renewed enthusiasm for open charm spectroscopy - in large because of the curious properties of the new state[131]. Fig. [29] shows fits to the $D_{s} \pi$ mass distributions in the $K K \pi \pi$ and $K K \pi \pi \pi$ modes. The resulting resonance masses are

$$
\begin{gathered}
M\left(D_{s}\right)=2316.8 \pm 0.4 \pm 3 \mathrm{MeV} \\
M\left(D_{s}\right)=2317.6 \pm 1.3 \mathrm{MeV}
\end{gathered}
$$



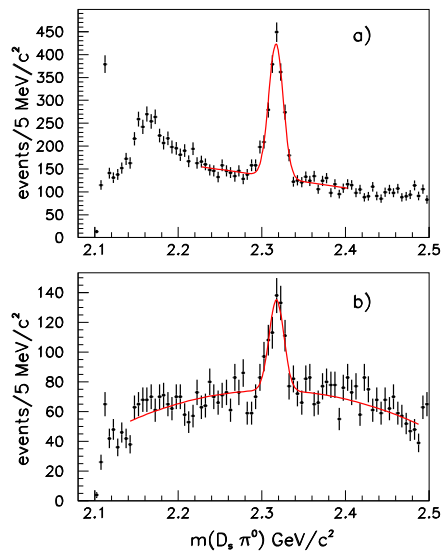

FIG. 29: $D_{s} \pi$ Mass Distribution for (a) $D_{s} \rightarrow K K \pi$ and (b) $D_{s} \rightarrow K K \pi \pi$.

respectively. The respective widths were determined to be $8.6 \pm 0.4 \mathrm{MeV}$ and $8.8 \pm 1.1 \mathrm{MeV}$. Presumably these are consistent with detector resolution since BaBar merely conclude that the width of the $D_{s}(2317)$ is less than $10 \mathrm{MeV}$ (no confidence interval is reported).

Evidently the new state is a $D_{s}$ meson which is decaying via an isospin violating mode. Since its mass is below the well-known $D_{s 1}(2536)$ and $D_{s 2}(2573)$, it suggests that the $D_{s}(2317)$ is a $J^{P}=0^{+}$state. This assignment disallows the decay mode $D_{s}(2317) \rightarrow D_{s} \gamma$, which is consistent with the lack of signal reported in this channel in Ref. [121]. Similarly, the $D_{s}(2317)$ was not seen in the $D_{s} \gamma \gamma$ decay mode.

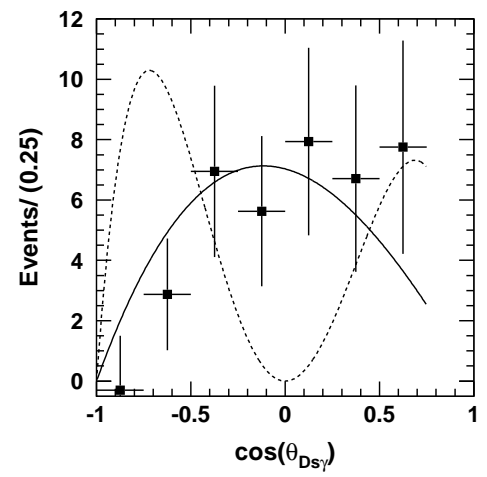

FIG. 30: The $D_{s}(2460) \rightarrow D_{s} \gamma$ Helicity Distribution. Solid and dashed lines are Monte Carlo predictions for the $J=1$ and $J=2$ hypotheses respectively 139$]$.

A search for the $D_{s}(2317)$ was also carried out in the $D_{s} \pi^{0} \gamma$ mode. No signal at $2317 \mathrm{MeV}$ was observed; although a peak at $2460 \mathrm{MeV}$ was commented on. The new state was subsequently confirmed by CLEO 132] in the $D_{s}^{*} \pi^{0}$ mode. Further data taking by BaBar [133] yielded the following improved mass measurements:

$$
M(D s(2317))=2318.9 \pm 0.3 \pm 0.9 \mathrm{MeV}
$$

and for the $D_{s}(2460)$ 134]:

$$
\begin{gathered}
M\left(D_{s}(2460) \rightarrow D_{s} \gamma\right)=2457.2 \pm 1.6 \pm 1.3 \mathrm{MeV} \\
M\left(D_{s}(2460) \rightarrow D_{s} \pi^{0} \gamma\right)=2459.1 \pm 1.3 \pm 1.2 \mathrm{MeV} \\
M\left(D_{s}(2460) \rightarrow D_{s} \pi^{+} \pi^{-}\right)=2460.1 \pm 0.3 \pm 1.2 \mathrm{MeV} .
\end{gathered}
$$


The combined result of the latter three measurements is

$$
M\left(D_{s}(2460)\right)=2459.4 \pm 0.3 \pm 1.0 \mathrm{MeV}
$$

As seen in Fig. [30] the $D_{s} \gamma$ helicity distribution strongly prefers a $J=1$ assignment for the $D_{s}(2460)[139]$. Thus the low-lying $D_{s}(P)$ quadruplet is complete.

The following ratios of branching fractions were also obtained:

$$
\begin{gathered}
\frac{B r\left(D_{s}(2460) \rightarrow D_{s} \gamma\right)}{B r\left(D_{s}(2460) \rightarrow D_{s} \pi^{0} \gamma\right)}=0.375 \pm 0.054 \pm 0.057 \\
\frac{B r\left(D_{s}(2460) \rightarrow D_{s} \pi^{+} \pi^{-}\right)}{B r\left(D_{s}(2460) \rightarrow D_{s} \pi^{0} \gamma\right)}=0.082 \pm 0.018 \pm 0.011 \\
\frac{B r\left(D_{s}(2460) \rightarrow D_{s} \gamma\right)}{\operatorname{Br}\left(D_{s}(2460) \rightarrow D_{s} \pi^{0}\right)}<0.17 \\
\frac{B r\left(D_{s}(2460) \rightarrow D_{s} \pi^{0}\right)}{\operatorname{Br}\left(D_{s}(2460) \rightarrow D_{s} \pi^{0} \gamma\right)}<0.11
\end{gathered}
$$

and

$$
\frac{B r\left(D_{s}(2460) \rightarrow D_{s}(2317) \gamma\right)}{B r\left(D_{s}(2460) \rightarrow D_{s} \pi^{0} \gamma\right)}<0.23
$$

The latter three are $95 \%$ C.L.

Recently, Belle 135, 136] has reported the observations of the new $D_{s}$ 's in $B$ decays. The $\mathrm{D}_{s 0}^{*}(2317)$ is seen in the process $\mathrm{B}^{0} \rightarrow \mathrm{D}_{s 0}^{*+}(2317) \mathrm{K}^{-}, \mathrm{D}_{s 0}^{*+}(2317) \rightarrow \mathrm{D}_{s}^{+} \pi^{0}$, and the $\mathrm{D}_{s 1}^{+}(2460)$ may have been seen (at a much lower rate) in a similar chain to $\mathrm{D}_{s}^{+} \gamma$. The reported branching fractions are

$$
\begin{gathered}
\operatorname{Br}\left(\mathrm{B}^{0} \rightarrow \mathrm{D}_{s 0}^{*+}(2317) \mathrm{K}^{-}\right) \cdot \operatorname{Br}\left(\mathrm{D}_{s 0}^{*+}(2317) \rightarrow \mathrm{D}_{s}^{+} \pi^{0}\right)=(4.4 \pm 0.8 \pm 0.6 \pm 1.1) \cdot 10^{-5}, \\
\operatorname{Br}\left(\mathrm{B}^{0} \rightarrow \mathrm{D}_{s 1}^{*+}(2460) \mathrm{K}^{-}\right) \cdot \operatorname{Br}\left(\mathrm{D}_{s 1}^{*+}(2460) \rightarrow \mathrm{D}_{s}^{+} \gamma\right)=\left(0.53 \pm 0.20 \pm 0.6_{-0.15}^{+0.16}\right) \cdot 10^{-5} .
\end{gathered}
$$

One may compare these rates to those for $B \rightarrow D_{s} K$ with a branching fraction of roughly $4 \cdot 10^{-5}$. These processes can occur via $W$ exchange or final state interactions. The tree diagram can also contribute to a four-quark component of the $D_{s}$. Assuming that the $D_{s} \pi^{0}$ mode dominates the width of the $D_{s}(2317)$ then implies that the ratio

$$
\frac{B r\left(\bar{B}^{0} \rightarrow D_{s}(\pi, K, D)^{-}\right)}{\operatorname{Br}\left(\bar{B}^{0} \rightarrow D_{s 0}(\pi, K, D)^{-}\right)} \approx 1 .
$$

This is in agreement with expectations for $c \bar{s}$ states 137 . Alternatively this ratio is expected to be 10 for multiquark states.

Finally, the reported yields for possible charge partners of the $D_{s}(2317)$ are $-28 \pm 25$ for $D_{s}^{0}(2317)$ and $-39 \pm 16$ for $D_{s}^{++}(2317)$. Thus tetraquark models (to be discussed below) of the $D_{s}(2317)$ appear to be ruled out. Also we note that angular analysis [138] of $D_{s 1}(2536) \rightarrow D^{*+} K_{S}$ indicates the presence of a large S-wave amplitude. This observation counters the notion that the narrow $j_{q}=3 / 2$ states decay in D-wave. 


\section{Models}

The $D_{s}(2317)$ lies approximately $160 \mathrm{MeV}$ below the majority of model computations. A selection of such model predictions and a summary of the experimental masses are given in Table XIV One sees good agreement between data and models for the $j_{q}=3 / 2$ multiplet and poor agreement for the $j_{q}=1 / 2$ multiplet. This is also illustrated in Fig. 31 The poor agreement calls into question the $c \bar{s}$ interpretation of the new $D_{s}$ states; however this interpretation has received support from light cone sum rule decay computations [140]. A comprehensive analysis of the new states in the $c \bar{s}$ model is given by Cahn and Jackson [141].

The majority of models listed in Table XIV predict a $D_{s 0}$ between 2400 and $2500 \mathrm{MeV}$. The model of Matsuki and Morii [148] is unique in predicting the $D_{s 0}$ and $D s 1$ masses approximately correctly. The model employs a FoldyWouthuysen nonrelativistic reduction of an assumed scalar confining interaction and vector Coulomb interaction ${ }^{13}$. It is difficult to isolate the cause of the good agreement - it may due to relativistic light quark kinematics or the novel assumed spin structure of the static interaction. Further predictions of this model are discussed in Sect. IVE Finally, Godfrey discusses using radiative transitions to test models 150 .

TABLE XIV: Theory and Experimental $D_{s}$ Masses

\begin{tabular}{lllll}
\hline$D_{s 0}$ & $D_{s 1}$ & $D_{s 1}^{\prime}$ & $D_{s 2}$ & ref \\
\hline \hline 2480 & 2550 & 2550 & 2590 & GK[122] \\
2380 & 2510 & 2520 & 2520 & ZOR[142] \\
2388 & 2521 & 2536 & 2573 & GJ[143] \\
2508 & 2569 & 2515 & 2560 & EGF[144] \\
2455 & 2502 & 2522 & 2586 & LNR[145] \\
2487 & 2605 & 2535 & 2581 & DE[146] \\
2339 & 2496 & 2487 & 2540 & MM[147] \\
\hline $2317.3 \pm 0.4 \pm 0.8$ & $2458.0 \pm 1.0 \pm 1.0$ & & BaBar[121] \\
$2317.2 \pm 0.5 \pm 0.9$ & $2456.5 \pm 1.3 \pm 1.3$ & & Belle[139] \\
$2318.5 \pm 1.2 \pm 1.1$ & $2463.6 \pm 1.7 \pm 1.2$ & CLEO[132] \\
$2317.4 \pm 0.9$ & $2459.3 \pm 1.3$ & $2535.35 \pm 0.342572 .4 \pm 1.5$ RPP[17] \\
\hline \hline
\end{tabular}

\section{Molecules}

Such a strong deviation from expectations point to exotic structure, such as $D K$ molecules, or to a deep misunderstanding of heavy-light hadrons.

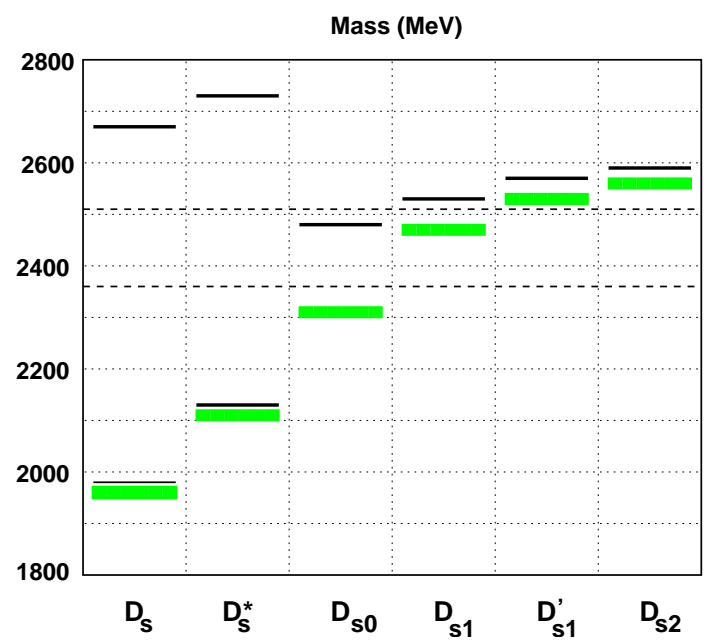

FIG. 31: The Low Lying $D_{s}$ Spectrum. Lines are theory from Ref. 18], boxes are experiment. The dashed lines show the $D K$ and $D^{*} K$ thresholds 26$]$.

13 The full vector current is used. Also, the authors found it was not possible to extract ${ }^{3} D_{1}$ and ${ }^{3} D_{2}$ masses, although recent computations have resolved this issue [149]. 
As can be seen in Fig. 31 the $D_{s 0}$ lies just below $D K$ threshold, while the $D_{s 1}$ lies just below $D K^{*}$. This suggests that mixing with these continua may be important components of the new $D_{s}$ states. Indeed, Barnes, Close, and Lipkin have suggested 151] that the $D_{s 0}$ and $D_{s 1}$ may be $D K^{(*)}$ molecules. They note that such an assignment naturally explains the anomalous masses of the $D_{s}(2317)$ and $D_{s}(2460)$. Furthermore, since the $D^{0} K^{+}$channel is roughly $9 \mathrm{MeV}$ higher than the $D^{+} K^{0}$ channel, isospin breaking is induced, thereby explaining the isospin breaking discovery mode of the $D_{s}(2317)$. The same observation holds for the $D K^{*}$ channel, suggesting that isospin breaking may be important in the $D_{s}(2460)$ as well.

The molecular assignment for the $D_{s 0}$ can be tested by measuring the radiative decay $D_{s 0} \rightarrow D_{s}^{*} \gamma$. A simple quark model computation gives $1 \mathrm{keV}$ for this E1 transition [26]. Similarly the $D_{s}(2460)$ transitions are $D_{s 1} \rightarrow D_{s} \gamma=$ $7.3 \sin ^{2} \phi \mathrm{keV}$ and $D_{s 1} \rightarrow D_{s}^{*} \gamma=4.2 \sin ^{2} \phi \mathrm{keV}$. Using the heavy quark mixing angle $\sin ^{2} \phi=2 / 3$ yields predicted rates of $4.5 \mathrm{keV}$ and $2.8 \mathrm{keV}$ respectively.

Alternatively, the radiative decay of molecular $D_{s J}$ proceeds via the annihilation diagram shown in Fig. 17 (right panel). A simple estimate of these rates gives 26.

$$
\begin{aligned}
& \Gamma\left(D_{s 0}(D K)\right.\left.\rightarrow D_{s}^{*} \gamma\right) \approx 35 \mathrm{keV} \\
& \Gamma\left(D_{s 1}\left(D K^{*}\right) \rightarrow D_{s}^{*} \gamma\right) \approx 45 \mathrm{keV} \\
& \Gamma\left(D_{s 1}\left(D K^{*}\right) \rightarrow D_{s} \gamma\right) \approx 50 \mathrm{keV}
\end{aligned}
$$

Thus it is possible that such a measurement will serve to distinguish the structure of the anomalous $D_{s}$ states. There is also the intriguing possibility of a radiative transition between molecular $D_{s 1}\left(D K^{*}\right)$ and $D_{s 0}(D K)$ states which would be driven by the virtual transition $K^{*} \rightarrow K \gamma$. This rate is estimated to be $17 \mathrm{keV}$ in Ref. [26], which may be compared to the analogous $c \bar{s}$ rate of $(0.3 \cos \phi+1.4 \sin \phi)^{2} \approx 1 \mathrm{keV}$. Recent computations of strong decay rates are presented in Ref. 152.

Finally, if the $D_{s 0}$ and $D_{s 1}$ really are molecular states, extra $D_{s J}$ states should appear in the spectrum around 2500 $\mathrm{MeV}$. Searching for these is of obvious importance.

Szczepaniak also considers a molecular assignment for the $D_{s}(2317)$ as $D_{s} \pi$ bound state with a binding energy of approximately $40 \mathrm{MeV}[153]$. He examines the properties of such a state with an effective lagrangian which incorporates the nonlinear realisation of chiral symmetry and $U_{A}(1)$ symmetry breaking via coupling of pions to the field $Q=F \tilde{F}$. The coupling of $D_{s}$ mesons to pions is affected via a $Q^{2} D_{s}^{2}$ term in the lagrangian. Parameters and cutoffs are fit to the mass of the $D_{s}(2317)$, and the formalism is used to predict other charged $D_{s} \pi^{ \pm}$modes, a scalar $D \pi$ at $2150-2300$ $\mathrm{MeV}$, and a scalar $D K$ molecule at $2440-2550 \mathrm{MeV}$. All of these molecules are predicted to have widths of order $10 \mathrm{MeV}$. Finally, Szczepaniak also suggests that $D^{*} \pi$ and $D^{*} K$ molecules may exist as well. Unfortunately, little evidence exists for this collection of predicted molecules. Generally, molecules containing pions are not considered viable because the small mass of the pion leads to very small molecular reduced masses, which tends to destabilise the system.

\section{Coupled Channels}

A less extreme version of the molecular picture postulates that the $D_{s}(2317)$ and $D_{s}(2460)$ are dominantly $c \bar{s}$ states which are heavily renormalised by mixing with the $D K$ and $D K^{*}$ continua. This approach has been followed by Hwang and Kim 154 who use the Cornell coupled channel model discussed in Sect. [B3 to compute the mass shifts experienced by bare $c \bar{s}$ states upon coupling to the $D K$ and $D K^{*}$ continua. The authors find that the bare scalar $D_{s}$ at $2480 \mathrm{MeV}$ has its mass lowered to $2320 \mathrm{MeV}$, in agreement with the $D_{s}(2317)$. The same philosophy has been followed by van Beveren and Rupp [155] except that the ${ }^{3} P_{0}$ model is used to drive the transitions between $c \bar{s}$ and the $D K$ channel. Note, however, that both papers do not consider coupling to other channels or renormalisation (see Sect. IB 4 for further discussion on this issue).

\section{Tetraquarks}

In general the $D_{s}$ has a Fock space expansion:

$$
D_{s}(2317)=Z_{1}^{1 / 2}|c \bar{s}\rangle+Z_{2}^{1 / 2}|c \bar{s}(u \bar{u}+d \bar{d})\rangle+Z_{3}^{1 / 2}\left|D^{0} K^{+}\right\rangle+Z_{4}^{1 / 2}\left|D^{+} K^{0}\right\rangle+\ldots
$$

The models presented here amount to different estimations of the relative strengths of the different channels. Tetraquark models assume $Z_{2} \gg Z_{1}, Z_{3}, Z_{4}$ and impose a specific structure on the internal configuration. Several groups have pursued this scenario $49,156,157,158,159$. Note that this is an old idea 160, 161] which builds on the proposed light tetraquarks of Jaffe $[51,162$.

A large spectrum of $[c q]\left[\bar{s} \bar{q}^{\prime}\right]$ tetraquark states exists and it is natural to attempt identifying some of these with the anomalous $D_{s}$ states. The $\left.[c q] \bar{s} \bar{q}^{\prime}\right]$ states may be obtained from Eq. 44 upon making the replacement $\bar{c} \rightarrow \bar{s}$. The resulting spectrum of Maiani et al. [49] is shown in Fig. [32 Possible assignments of the $D_{s}(2317), D_{s}(2460)$, and $D_{s}(2632)$ (to be discussed in the following section) are also indicated. 


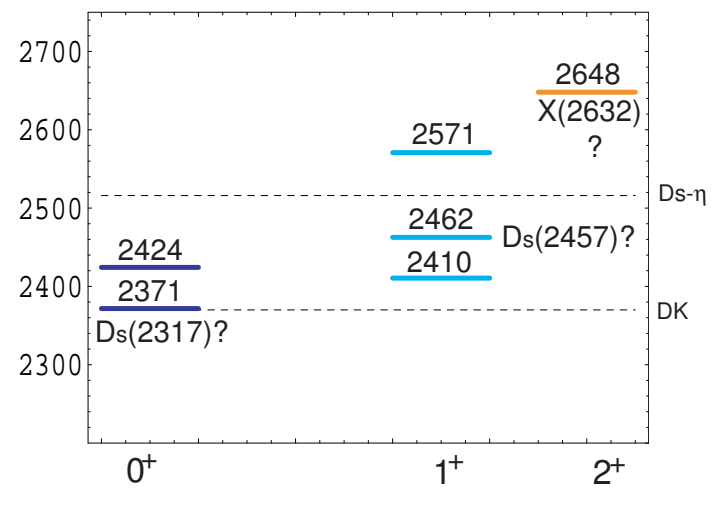

FIG. 32: $c \bar{s}$ Tetraquark Spectrum[49].

Along these lines, Chen and Li[163] suggest modified four quark configurations of $c \bar{s}(u \bar{u}+d \bar{d})$ for the $D_{s}(2317)$ and $D_{s}(2460)$. Nussinov [164] suggests that if the $D_{s}(2317)$ is a four quark state then a new isoscalar $D \bar{D}$ bound state with mass less than $3660 \mathrm{MeV}$ must exist.

Browder et al. 165 present a modified version of the tetraquark scenario, by assuming that tetraquark states lie above $2400 \mathrm{MeV}$ and mix with bare $c \bar{s}$ states at a similar mass. In this picture, the $D_{s}(2317)$ and $D_{s}(2460)$ are predominantly $c \bar{s}$ states and narrow, while the four-quark states lie above $D K$ threshold and are broad.

Lattice

UKQCD 166] have computed the $D_{s}$ spectrum in quenched and $N_{f}=2$ unquenched configurations. Their results are shown in Fig. 33] The figure illustrates that it is difficult to come to definitive conclusions on masses in this sector due to statistical errors of order $100 \mathrm{MeV}$ and systematic errors due to unquenching of similar size. Notice that the $D_{s 0}$ mass is approximately $100 \mathrm{MeV}$ higher than experiment - in keeping with naive quark model estimates (see the discussion in Sect. [B 4). We remark that it is extremely unlikely that the lattice can differentiate the $D_{s 1}$ and $D_{s 1}^{\prime}$ states. Similar results are also reported by Bali 167], who notes that significant finite heavy quark mass corrections to the heavy quark mass limit are present at the charm mass scale, non-degenerate sea quarks may be required, and quenched simulations of four-quark states are desirable.

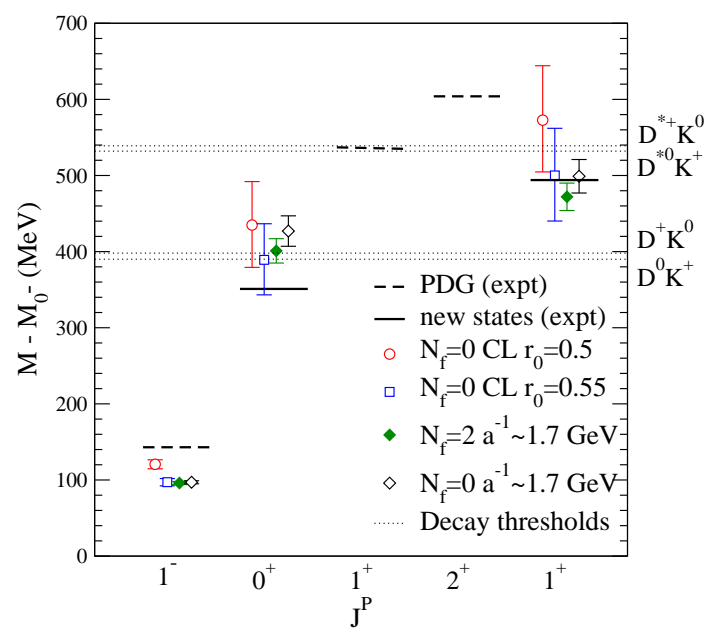

FIG. 33: Lattice $D_{s}$ Spectrum 166].

\section{Summary}

It appears likely that the $j_{q}=1 / 2\left(D_{s 0}, D_{s 1}\right)$ multiplet is difficult to interpret as simple $c \bar{s}$ states and novel dynamics are required. Of the continuum of possibilities, tetraquarks are the most speculative and least constrained by theory and experiment. Because of the unknown dynamics, tetraquark models tend to predict extensive spectra. So far there 
is little evidence for tetraquark supernumerary states and one must regard these models as unestablished.

Molecular interpretations are similar to tetraquark in the assumption that four quark configurations dominate the $D_{s 0}$ and $D_{s 1}$. However the presumed diffuse spatial configurations are very different from those of tetraquark clusters (of course, the colour and spin configurations can vary as well). Sufficiently assiduous experimental study will be able to distinguish these scenarios. Typical molecular models also suffer from poor knowledge of the relevant dynamics in this case the nonperturbative gluodynamics that drives meson coupling to the continuum. Thus, for example, if S-wave channels dominate the putative $D K$ molecule one expects no charged partners. However, these can exist if the $D K$ binding is driven by t-channel exchange.

The scenario which requires the weakest extension of the naive quark model assumes that mixing of scalar (and axial) bare $c \bar{s}$ states to the $D K$ (and $D K^{*}$ ) continua substantially modify the structure of these states. This mechanism is thought to be responsible for the anomalous properties of the $f_{0}(975)$ and $a_{0}(980)$ and in general, should be important whenever bare quark states couple in S-wave to nearby continua. I regard this scenario as the most likely explanation of the new $D_{s}$ states.

Unfortunately, theoretical progress will be difficult due to the previously mentioned unknown gluodynamics which drives Fock sector mixing, and the difficulty in consistently renormalising the quark model. But it is becoming clear that this effort will be required to understand a slowly expanding subset of the hadronic spectrum.

\section{D. $D_{s}(2630)$}

The $D_{s}(2630)$ was discovered by the SELEX collaboration at FNAL[168] in the final states $D^{0} K^{+}$and $D_{s} \eta$. The peak in the $D K$ mode can be seen in Fig. 34] The combined measured mass is $2632.6 \pm 1.6 \mathrm{MeV}$ and the state is surprisingly narrow with a width of less than $17 \mathrm{MeV}$ at the $90 \%$ confidence level.

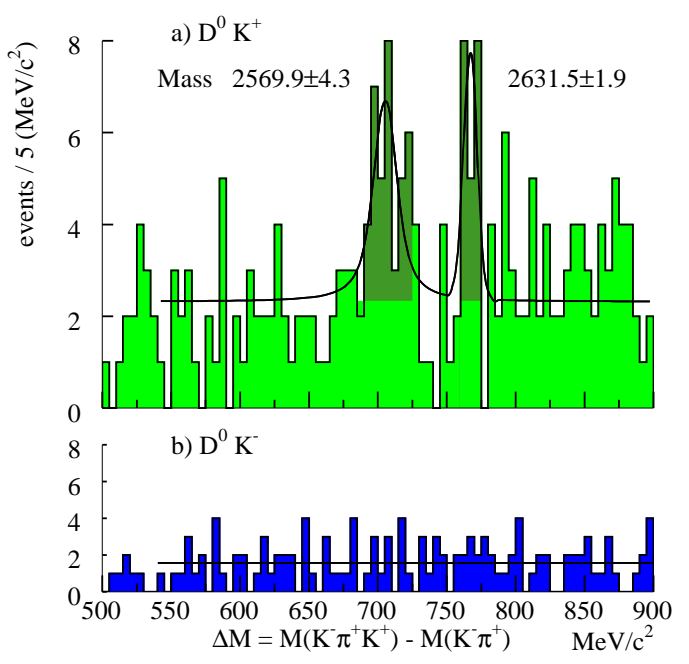

FIG. 34: Observation of the $D_{s}(2630)$ in the $D K$ Mode 168].

The ratio of the partial widths is measured to be

$$
\frac{\Gamma\left(D_{s} \rightarrow D^{0} K^{+}\right)}{\Gamma\left(D_{s} \rightarrow D_{s} \eta\right)}=0.16 \pm 0.06 .
$$

As pointed out by the SELEX collaboration, this is an unusual result since the $D K$ mode is favoured by phase space.

This observation has engendered speculation that the $D_{s}(2630)$ is an exotic state. It is unlikely that this state is a $c \bar{s}$ hybrid since the mass of such a hybrid is expected to be roughly $3170 \mathrm{MeV}$. Possible molecular states include a $D_{s}^{*} \eta$ system at $2660 \mathrm{MeV}$ or $D_{s}^{*} \omega$ or $D^{*} K^{*}$ states at $2900 \mathrm{MeV}$. However the former is a P-wave which is not favoured for binding, while the latter are too high in mass to be plausible.

The large number of possible tetraquarks makes it simple to fit the $D_{s}(2630)$ into tetraquark spectra. For example, Chen and $\mathrm{Li}\left[137\right.$ regard the new $D_{s}$ as a $[c s][\bar{s} \bar{s}]$ state - an $\mathrm{SU}(3)$ partner to the $D_{s}(2317)$. Claimed destructive interference in the decay amplitude leads to a narrow width for the state. The authors predict an axial-vector 
tetraquark partner of the $D_{s}(2630)$ with a mass $M\left(D_{s 1}\right) \approx 2770 \mathrm{MeV}$. Note that the maximally attractive tetraquark channel, which is antisymmetric in colour and in spin, is forbidden because of the identical anti-strange quarks [169.

Alternatively, Maiani et al. suggest [170] that the $D_{s}(2630)$ is a $[c d][\bar{d} \bar{s}]$ tetraquark (see Fig. [32). They speculate that this state does not mix with $[c u][\bar{u} \bar{s}]$ and hence breaks isospin symmetry. The decay to $D^{0} K^{+}$would then be OZI suppressed. However the state should decay to $D_{s} \pi^{0}$ and $D^{+} K^{0}$ with sizable branching fraction. Discovering the $D_{s} \pi$ mode (along with the observed $D_{s} \eta$ mode) would indicate isospin breaking, as required in this scenario. Maiani et al. also predict the existence of a charge +2 state with mass near $2630 \mathrm{MeV}$, which decays to $D_{s} \pi^{+}$and $D^{+} K^{+}$.

Finally, van Beveren and Rupp[171] suggest that the $D_{s}(2630)$ is a heavily renormalised $c \bar{s}$ state with the aid of a $\mathrm{K}$ matrix coupled channel model. The model also predicts $D_{s}^{* \prime}$ states at 2720,3030 , and $3080 \mathrm{MeV}$ with widths of 2, 50-60, and $8 \mathrm{MeV}$ respectively.

If the predicted decay modes or partner states of the $D_{s}$ are not found, the only remaining possibility is that the $D_{s}(2630)$ is a radially excited $c \bar{s}$ vector 172 (although it is some $100 \mathrm{MeV}$ lighter than the quark model predictions of $2730 \mathrm{MeV})$. The peculiar decay ratio remains to be explained. Experience with the decay modes of the $\psi(3 S)$ to $D D, D D^{*}$ and $D^{*} D^{*}$ points to a possible resolution: transition matrix elements for excited hadrons may have zeroes due to wavefunction nodes. It is possible that such a node is suppressing the $D K$ decay mode. Computation [169], shown in Fig. 35] reveals that there is indeed a node but that it occurs at a wavefunction scale which is $20 \%$ lower than the scale expected in quark models. Furthermore, the $D K$ mode is always larger than the $D_{s} \eta$ mode so that explaining the data is difficult in this scenario.

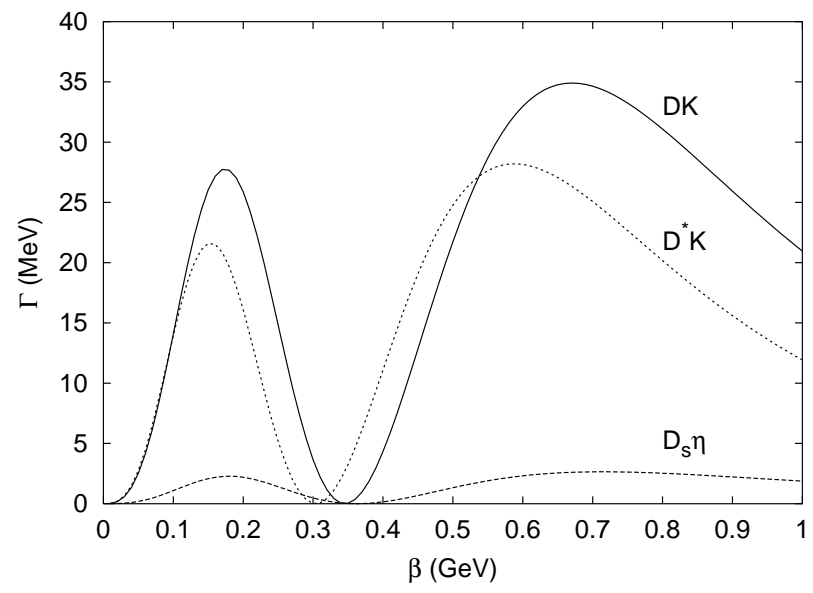

FIG. 35: Theoretical Partial Widths for the $D_{s}(2630)$ as a Function of the SHO $D_{s}$ Scale $\beta$ [169].

We have run out of options and must conclude that the $D_{S}(2630)$ is an experimental artefact. This conclusion now appears likely because searches by FOCUS [173], BaBar 174] (see Fig. [36), and CLEO [175] have found no evidence for the $D_{s}(2630)$.

\section{E. P-wave $B$ Mesons}

Preliminary results from $\mathrm{CDF}$ and $\mathrm{D} \varnothing$ on orbitally excited $\mathrm{B}$ mesons were presented at Hadron 05 [177]. The $B$ mesons were reconstructed in $J / \psi K \pi$ and sufficient events were collected to discern the $B_{2}$ and $B_{1}$ contributions to the rate (see Fig. 37).

The DØ mass results are

$$
\begin{gathered}
M\left(B_{1}\right)=5724 \pm 4 \pm 7 \mathrm{MeV}, \\
M\left(B_{2}\right)-M\left(B_{1}\right)=23.6 \pm 7.7 \pm 3.9 \mathrm{MeV} .
\end{gathered}
$$

Godfrey and Isgur [18] predict $M\left(B_{2}\right)=5800 \mathrm{MeV}, 52 \pm 12 \mathrm{MeV}$ too high. The $B_{1}$ s are predicted to be "within 40 $\mathrm{MeV}$ of the $B_{2}$ ". Alternatively, the model of Matsuki and Morii 147], which was so noticeably successful for the $D_{s}$ system, predicts $M\left(B_{2}\right)=5692 \mathrm{MeV}$ and $M\left(B_{1}\right)=5740$ or $5679 \mathrm{MeV}$.

The total widths, constrained in the fit to be equal (and predicted to be comparable theoretically) are

$$
\Gamma=23 \pm 12 \mathrm{MeV}
$$




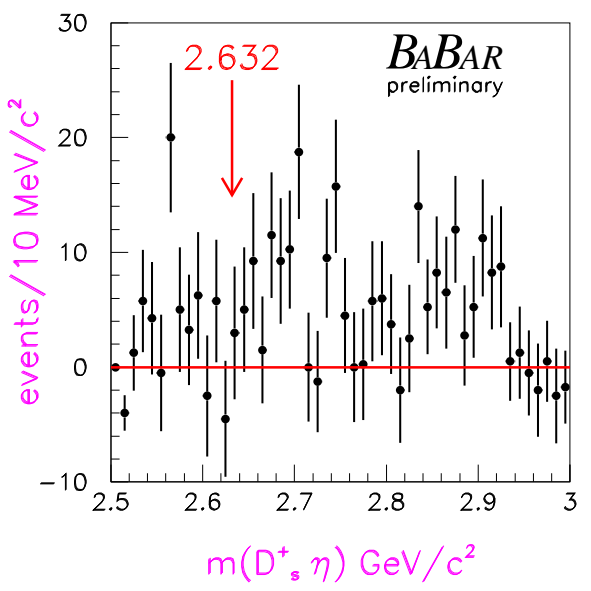

FIG. 36: Non-observation of the $D_{s}(2630)$ in the $D_{s} \eta$ Mode by BaBar 174].

which is consistent with theoretical expectations for the two narrow $\mathrm{P}$-wave $\mathrm{B}$ mesons. The experimental resolution is estimated to be about $10 \mathrm{MeV}$.

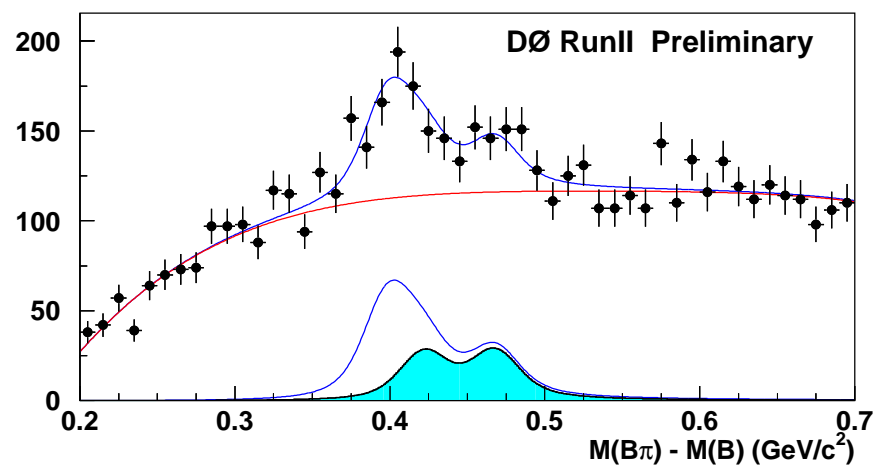

FIG. 37: Orbitally-excited P-wave B mesons, as reported by DØ. Fitted contributions from $\mathrm{B}_{1} \rightarrow \mathrm{B} \pi, \mathrm{B}_{2}^{*} \rightarrow \mathrm{B} \pi$ and $\mathrm{B}_{2}^{*} \rightarrow \mathrm{B}^{*} \pi$ are shown [178].

F. $\quad B_{c}$

The $B_{c}$ pseudoscalar meson was originally seen in the semileptonic decay channels $B_{c} \rightarrow J / \psi \ell \nu_{\ell} X$ at FNAL[179]. Unfortunately the low statistics and unreconstructed final state did not permit an accurate determination of the mass:

$$
M\left(B_{c}\right)=6400 \pm 390 \pm 130 \mathrm{MeV} .
$$

In May 2005 the CDF collaboration [180] announced an accurate determination of the $B_{c}$ mass. The state was seen in $14.6 \pm 4.6$ events in the $J / \psi \pi^{ \pm}$mode (see Fig. (38) with a reported mass of

$$
M\left(B_{c}\right)=6285.7 \pm 5.3 \pm 1.2 \mathrm{MeV} .
$$




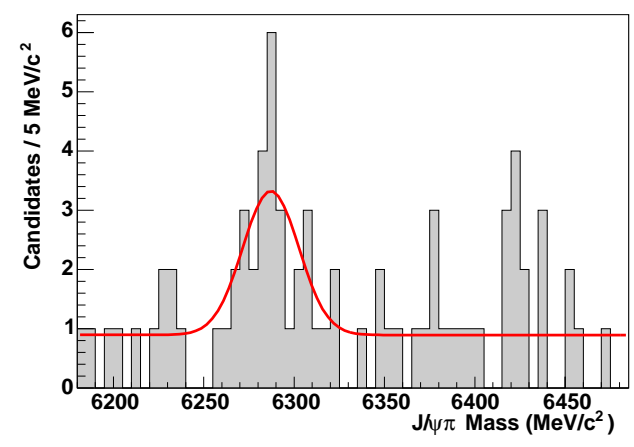

FIG. 38: $B_{c}$ Signal [180].

Subsequently, the CDF collaboration has also reconstructed [181] the $B_{c}$ in the decay mode $B_{c} \rightarrow J / \psi \ell$ with a significance of $5.2 \sigma$ for $\ell=\mu$ (or $5.9 \sigma$ for $\ell=e$ ). The following ratios of branching fractions have been measured:

$$
\frac{\sigma\left(B_{c}\right) B r\left(B_{c} \rightarrow J / \psi \mu\right)}{\sigma(B) B r(B \rightarrow J / \psi K)}=0.249 \pm 0.045_{-0.076}^{+0.107}
$$

and

$$
\frac{\sigma\left(B_{c}\right) B r\left(B_{c} \rightarrow J / \psi e\right)}{\sigma(B) B r(B \rightarrow J / \psi K)}=0.282 \pm 0.038 \pm 0.074
$$

An updated fit to the $B_{c} \rightarrow J / \psi \pi$ decay mode yields 181

$$
M\left(B_{c}\right)=6287.0 \pm 4.8 \pm 1.1 \mathrm{MeV} .
$$

Lastly, the lifetime of the $B_{c}$ has been measured to be

$$
\tau\left(B_{c}\right)=0.474_{-0.066}^{+0.073} \pm 0.033 \mathrm{ps} .
$$

This may be compared to theoretical expectations [182] of $0.55 \pm 0.15$ ps. Remarkably, the inclusive computation of this lifetime considers 65 different decay modes.

The timing of the CDF announcement was arranged with the HPQCD lattice group 183 to permit them a prediction of its mass. The lattice prediction was $6304 \pm 12_{-0}^{+18} \mathrm{MeV}$. Thus CDF may claim a state, the lattice may claim a victory, and the $B_{c}$ appears to carry no surprises. While there is justifiable pride in the present predictions and experiment, some perspective may be gained when it is recalled that Godfrey and Isgur predicted a $B_{c}$ of $6270 \mathrm{MeV}$ some twenty years ago [18]. As stated in Sect. [B 4 this should not astonish, the quark model and lattice gauge theory tend to agree on states with a simple Fock space expansion.

\section{CONCLUSIONS}

The moderately heavy charm quark makes charming spectroscopy especially interesting since it lends confidence that the behaviour of soft QCD can be extracted from the data, yet it is not so heavy that one is simply exploring an analogue of the positronium spectrum. Thus the new charming mesons have generated much enthusiasm. The $X(3872), D_{s}(2317)$, and $D_{s}(2460)$ remain enigmatic and highlight a less than perfect understanding of QCD. Can we be seeing the expected breakdown of the naive quark model? Where and how do gluonic degrees of freedom manifest themselves? Have we entered a new era of 'mesonic nuclear physics'?

If the $X(3872)$ is not canonical charmonium then the simplest explanation lies in expanding the Fock space description of the state. With $1^{++}$quantum numbers this is likely to mean $c \bar{c} q \bar{q}$. Whether a diffuse four-quark state dominates the $X$, as in the molecular interpretation, or a compact state dominates as with tetraquarks, or the fourquark component heavily renormalises an erstwhile $\chi_{c 1}^{\prime}$ is a matter of dynamics. This dynamics can be subtle, but 
often leads to different and testable predictions. Thus, for example, an entire spectrum of tetraquarks is predicted while a modest number of additional molecular states are expected. Alternatively, the idea that the $X$ is a purely kinematical cusp effect can be checked by measuring the rate for $B^{+} \rightarrow K^{0} D^{+} \bar{D}^{* 0}$. If no enhancement is seen in this channel it implies that significant final state interactions are creating the $X$ signal. Finally, pinning down the $D D \pi$ mode will be vital in establishing the magnitude of $c \bar{c}-c \bar{c} q \bar{q}$ mixing. And this will tell us something about the gluodynamics which generates mixing.

The $X, Y, Z$ charmonium complex near $3940 \mathrm{MeV}$ poses interesting problems. Prima facie, it has some of the expected features of a $2 \mathrm{P}$ multiplet. However, if the $Z$ is interpreted as the $\chi_{c 2}^{\prime}$ and the $X(3940)$ is the $\chi_{c 1}^{\prime}$ then the multiplet displays an inverted spin orbit splitting. This unusual situation would then highlight significant confusion in our understanding of the Dirac structure of confinement. Alternatively, the unusual $\omega J / \psi$ decay mode of the $Y(3940)$ has led to suggestions that it is a charmonium hybrid. This suggestion does fit with (rather shaky) expectations for hybrid decays; however, the mass is much too low compared to (somewhat less shaky) quenched lattice computations and prudence - and more data - are called for. The evidence for the $Y(3940)$ is not especially robust and one must hope for additional experimental effort in determining the properties of this state. Lastly, if the $X(3940)$ is the $\chi_{c 1}^{\prime}$ then why is the $\chi_{c 1}$ not apparent in the same data set?

The $Y(4260)$ is an intriguing candidate for a vector charmonium hybrid. This possibility may be pursued by searching for other $S+P$ decay modes such as $\chi_{c 0} \omega$ and for nearby pseudoscalar and $J^{P C}=1^{-+}$hybrids. The resonance interpretation of the $Y$ may be confounded by the proximity of the $D_{1} \bar{D}$ channel which will cause an enhancement due to the threshold cusp effect and possible strong $D_{1} \bar{D}$ rescattering. This possibility can be tested by measuring analogous strange systems such as $D_{s 1} \bar{D}$ and $D_{s 1} \bar{D}_{s}$. Finding manifestations of glue in the spectrum is a primary goal of nonperturbative QCD and one must hope that the $Y$ will be studied in greater detail. CLEO-c may be able to assist substantially in this effort.

The masses of the $D_{s 0}(2317)$ and $D_{s 1}(2460)$ point to a significant lack of understanding of QCD dynamics in the $c \bar{s}$ system. Possible interpretations include tetraquark states or molecular $D K$ or $D K^{*}$ states. The scenario which requires the weakest extension of the naive quark model assumes that mixing of scalar (and axial) bare $c \bar{s}$ states to the $D K$ (and $D K^{*}$ ) continua substantially modify the structure of these states. An alternative explanation is provided by the chiral doublet model, but it has been argued that this model is likely not relevant to low-lying heavy-light states in Section IVB2 Firmly establishing the analogous $D$ states will assist in understanding the novel dynamics which appears to be present in $P$-wave heavy-light hadrons [184].

The advent of the $\Theta$ pentaquark states and the new mesons has led to increased interest in diquark correlations as effective degrees of freedom. However, the subsequent collapse of the pentaquark states raises serious concerns about the reliability of chiral soliton and other pentaquark models. Similarly, an infinite collection of tetraquark states has been predicted. However, there is no evidence for any of the predicted low lying states. Clearly a degree of circumspection is called for.

Alternatively, many of the proffered explanations of the unusual states involve mixing with higher Fock components. These explanations are economical in that new dynamics or degrees of freedom need not be postulated. However, they must be carefully constructed because it is relatively easy to destroy previous agreement with experiment. A simple organising principle seems to be emerging: the presence of nearby S-wave meson-meson thresholds can strongly affect meson structure. While this observation can serve as a useful rule of thumb, turning it into a quantitative theory is a daunting task because it is driven by unknown nonperturbative gluodynamics. Of course, understanding gluodynamics is one of the great remaining challenges in the development of the Standard Model, and the effort is worthwhile.

More than a decade ago Isgur stated [185], "Twenty five years after the birth of the quark model, light quark ... spectroscopy is in a deplorable state". While the situation is slowly improving, it is the formerly comfortable heavy quark sector which is now under siege. And new data seem to reveal new ignorance with each passing month. It is clear that the simple constituent quark model must fail somewhere - are we seeing this? The new heavy meson spectroscopy challenges our understanding of QCD. Can we meet the challenge?

\section{Acknowledgments}

I am grateful to the organisers of the International Conference on QCD and Hadronic Physics and the International Conference on Hadron Spectroscopy for invitations to speak about this fascinating topic. These talks formed the nucleus of this review. I would like to thank Ted Barnes, Denis Bernard, Eric Braaten, Frank Close, Alexey Petrov, Walter Toki, Steve Dytman, Steve Godfrey, Steve Olsen, and Steve Yzerman for inspiration and many interesting discussions on heavy hadron phenomenology. This work was supported by the US Department of Energy under contract DE-FG02-00ER41135, the National Science Foundation under grant NSF-PHY-244668, and PPARC grant 
$\mathrm{PP} / \mathrm{B} 500607$ in the UK.

[1] C. Quigg, "Hadronic Physics and Exotics", hep-ph/0509332

[2] T. Appelquist and H.D. Politzer, Phys. Rev. Lett. 34, 43 (1975); A. De Rujula and S.L. Glashow, Phys. Rev. Lett. 34, $46(1975)$

[3] E. Eichten, K. Gottfried, T. Kinoshita, K. D. Lane and T. M. Yan, Phys. Rev. D 17, 3090 (1978) [Erratum-ibid. D 21, 313 (1980)]; Phys. Rev. D 21, 203 (1980).

[4] M. E. Peskin, Nucl. Phys. B 156, 365 (1979). For earlier related work see K. Gottfried, Phys. Rev. Lett. 40, 598 (1978) and H. Goldberg, Phys. Rev. Lett. 35, 605 (1975).

[5] G. T. Bodwin, E. Braaten and G. P. Lepage, Phys. Rev. D 51, 1125 (1995) [Erratum-ibid. D 55, 5853 (1997)].

[6] A. Pineda and F. J. Yndurain, Phys. Rev. D 58, 094022 (1998); N. Brambilla, A. Pineda, J. Soto and A. Vairo, Nucl. Phys. B 566, 275 (2000); N. Brambilla et al., "Heavy quarkonium physics", arXiv:hep-ph/0412158 N. Brambilla, "QCD Effective Field Theories for Heavy Quarkonium", arXiv:/hep-ph/0510194.

[7] G. P. Lepage, "How to renormalize the Schroedinger equation", arXiv:nucl-th/9706029

[8] E. Eichten and F. Feinberg, Phys. Rev. D23, 2724 (1981). For earlier work see L. S. Brown and W. I. Weisberger, Phys. Rev. D20, 3239 (1979).

[9] J. T. Pantaleone, S. H. H. Tye and Y. J. Ng, Phys. Rev. D 33, 777 (1986).

[10] D. Gromes, Z. Phys. C11, 147 (1981); erratum C14, 94 (1982).

[11] H.J. Schnitzer, Phys. Rev. Lett. 35, 1540 (1975).

[12] W. Lucha, F. F. Schöberl, and D. Gromes, Phys. Rep. 200, 127 (1991).

[13] T. Barnes, S. Godfrey and E. S. Swanson, Phys. Rev. D72, 054026 (2005).

[14] D. Ebert, R. N. Faustov and V. O. Galkin, Phys. Rev. D 67, 014027 (2003) arXiv:hep-ph/0210381. A fully relativistic computation is presented in D. Ebert, R. N. Faustov and V. O. Galkin, Mod. Phys. Lett. A 20, 875 (2005) arXiv:hep-ph/0503012.

[15] M. Okamato et al. [CP-PACS], Phys. Rev. D65, 094508 (2002).

[16] P. Chen, Phys. Rev. D64, 034509 (2001).

[17] S. Eidelman et al. [RPP], Phys. Lett. B592, 1 (2004).

[18] S. Godfrey and N. Isgur, Phys. Rev. D 32 (1985) 189.

[19] W. Buchmüller, Phys. Lett. 112B, 479 (1982). For extensions of this idea see M.G. Olsson, S. Veseli, and K. Williams, Phys. Rev. D53, 4006 (1996) and references therein.

[20] W. J. Lee and D. Weingarten, Phys. Rev. D 61, 014015 (2000) arXiv:hep-lat/9910008; C. McNeile, C. Michael and G. Thompson [UKQCD Collaboration], Phys. Rev. D 70, 054501 (2004) arXiv:hep-lat/0404010; R. D. Loft and T. A. DeGrand, Phys. Rev. D 39, 2692 (1989); see also Ref. [119].

[21] L. Micu, Nucl. Phys. B10, 521 (1969); E. W. Colglazier and J. L. Rosner, Nucl. Phys. B 27, 349 (1971); W. P. Petersen and J. L. Rosner, Phys. Rev. D 6 (1972) 820; A. Le Yaouanc, L. Oliver, O. Pène and J.-C. Raynal, Phys. Rev. D8, (1973) 2223; A. Le Yaouanc, L. Oliver, O. Pène and J.-C. Raynal, Phys. Rev. D9, 1415 (1974); R. Kokoski and N. Isgur, Phys. Rev. D35, 907 (1987).

[22] E. S. Ackleh, T. Barnes and E. S. Swanson, Phys. Rev. D 54, 6811 (1996).

[23] P. Geiger and E. S. Swanson, Phys. Rev. D 50, 6855 (1994).

[24] C. Quigg, Nucl. Phys. Proc. Suppl. 142, 87 (2005).

[25] K. K. Seth, "A new analysis of the R measurements: Resonance parameters of the higher vector states of charmonium", arXiv:hep-ex/0405007

[26] F. E. Close and E. S. Swanson, Phys. Rev. B72, 094004 (2005).

[27] E. S. Swanson, J. Phys. G 31, 845 (2005).

[28] T. D. Cohen and L. Y. Glozman, Phys. Rev. D 65, 016006 (2002); E. S. Swanson, Phys. Lett. B 582, 167 (2004).

[29] A. P. Szczepaniak and E. S. Swanson, Phys. Rev. D 55, 3987 (1997).

[30] A. P. Szczepaniak and E. S. Swanson, Phys. Rev. Lett. 87, 072001 (2001).

[31] A. Le Yaouanc, L. Oliver, S. Ono, O. Pene and J. C. Raynal, Phys. Rev. D 31, 137 (1985); N. Ligterink and E. S. Swanson, Phys. Rev. C 69, 025204 (2004); F. J. Llanes-Estrada, S. R. Cotanch, A. P. Szczepaniak and E. S. Swanson, Phys. Rev. C 70, 035202 (2004); P. Maris and C. D. Roberts, Int. J. Mod. Phys. E 12, 297 (2003).

[32] S.-K. Choi et al. [Belle Collaboration], Phys. Rev. Lett. 91, 262001 (2003).

[33] D. Acosta et al. [CDF II Collaboration], Phys. Rev. Lett. 93, 072001 (2004).

[34] V. M. Abazov et al. [DØ Collaboration], Phys. Rev. Lett. 93, 162002 (2004) arXiv:hep-ex/0405004.

[35] B. Aubert et al. [BaBar Collaboration], Phys. Rev. D 71, 071103 (2005).

[36] CDF Note 7570. An updated analysis is presented in A. Abulencia, D. Acosta and J. Adelman, arXiv:hep-ex/0512074

[37] B. Aubert et al. [BABAR Collaboration], Phys. Rev. Lett. 93, 041801 (2004).

[38] B. Aubert et al. [BaBar Collaboration], Phys. Rev. D 71, 031501 (2005).

[39] D. Bernard, talk at International Conference on QCD and Hadronic Physics, Beijing, June 2005. See also [110].

[40] D. Bernard, private communication.

[41] B. Aubert et al. [BaBar Collaboration], Phys. Rev. D 71, 052011, (2005). 
[42] E. S. Swanson, Phys. Lett. B 598, 197 (2004).

[43] K. Abe et al., "Evidence for X(3872) $\rightarrow$ gamma J/psi and the sub-threshold decay X(3872) $\rightarrow$ omega J/psi", arXiv:hep-ex/0505037 K. Abe et al. [Belle Collaboration], "Properties of the X(3872) at Belle", arXiv:hep-ex/0408116 BELLE-CONF-0540.

[44] K. Abe et al., "Experimental constraints on the possible J(PC) quantum numbers of the X(3872)", arXiv:hep-ex/0505038

[45] G. Bauer, arXiv:hep-ex/0505083 BELLE-CONF-0568; S. Olsen, private communication.

[46] C. Z. Yuan, X. H. Mo and P. Wang, Phys. Lett. B 579, 74 (2004).

[47] G. Bauer [CDF II Collaboration], Int. J. Mod. Phys. A 20, 3765 (2005) arXiv:hep-ex/0409052.

[48] T. Barnes and S. Godfrey, Phys. Rev. D 69, 054008 (2004).

[49] L. Maiani, F. Piccinini, A. D. Polosa and V. Riquer, Phys. Rev. D 71, 014028 (2005).

[50] D. Ebert, R. N. Faustov and V. O. Galkin, arXiv:hep-ph/0512230

[51] For early work on diquarks see R. L. Jaffe, Phys. Rev. D 15, 267 (1977).

[52] M. Karliner and H. J. Lipkin, arXiv:hep-ph/0601193

[53] B. A. Li, Phys. Lett. B 605, 306 (2005).

[54] X. Liao and T. Manke, "Excited charmonium spectrum from anisotropic lattices", arXiv:hep-lat/0210030

[55] R. Giles and S. H. H. Tye, Phys. Rev. Lett. 37, 1175 (1976); T. Barnes, Caltech PhD thesis (1977); D. Horn and J. Mandula, Phys. Rev. D 17, 898 (1978); N. Isgur and J. Paton, Phys. Rev. D 31, 2910 (1985); T. Barnes, F. E. Close and E. S. Swanson, Phys. Rev. D 52, 5242 (1995).

[56] K. K. Seth, Phys. Lett. B 612, 1 (2005).

[57] H. Hogaasen, J. M. Richard and P. Sorba, arXiv:hep-ph/0511039

[58] Y. S. Kalashnikova, Phys. Rev. D 72, 034010 (2005).

[59] D. V. Bugg, Phys. Lett. B 598, 8 (2004).

[60] Molecular $\psi(4040)$ : M.B. Voloshin and L.B. Okun, JETP Lett. 23, 333 (1976); A. de Rujula, H. Georgi, and S.L. Glashow, Phys. Rev. Lett. 38, 317 (1977). Molecular $a_{0}(908)$ : J. D. Weinstein and N. Isgur, Phys. Rev. D 41, 2236 (1990). Molecular $f_{1}(1420)$ : D.G. Caldwell, Mod. Phys. Lett. A2, 771 (1987); R.S. Longacre, Phys. Rev. D42, 874 (1990); Annals Phys. 220, 73 (1992). Molecular $\eta(1440)$ : see 61] Molecular $f_{J}(1720)$ : K. Dooley, E. S. Swanson and T. Barnes, Phys. Lett. B 275, 478 (1992).

[61] N. A. Törnqvist, Z. Phys. C 61, 525 (1994).

[62] N. A. Törnqvist, "Comment on the narrow charmonium state of Belle at $3871.8 \mathrm{MeV}$ as a deuson", arXiv:hep-ph/0308277

[63] F. E. Close and P. R. Page, Phys. Lett. B 578, 119 (2004).

[64] C. Y. Wong, Phys. Rev. C 69, 055202 (2004).

[65] S. Pakvasa and M. Suzuki, Phys. Lett. B 579, 67 (2004).

[66] E. S. Swanson, Phys. Lett. B 588, 189 (2004);

[67] T. W. Chiu and T. H. Hsieh [TWQCD Collaboration], arXiv:hep-ph/0603207

[68] G.S. Bali, Phys. Rev. D62, 114503 (2000).

[69] A.M. Green, J. Koponen, and P. Pennanen, Phys. Rev. D61, 014014 (2000).

[70] E. S. Swanson, Annals Phys. 220, 73 (1992); T. Barnes and E. S. Swanson, Phys. Rev. D 46, 131 (1992); E. S. Swanson, "Hadron-hadron interactions in the constituent quark model: results and extensions", arXiv:hep-ph/0102267

[71] T. Barnes and E. S. Swanson, Phys. Rev. C 49, 1166 (1994);

[72] C. Y. Wong, E. S. Swanson and T. Barnes, Phys. Rev. C 65, 014903 (2002) [Erratum-ibid. C 66, 029901 (2002)]; C. Y. Wong, E. S. Swanson and T. Barnes, Phys. Rev. C 62, 045201 (2000).

[73] S. Weinberg, Nucl. Phys. B363, 3 (1991).

[74] M. B. Voloshin, Phys. Lett. B 579, 316 (2004).

[75] E. Braaten and M. Kusunoki, Phys. Rev. D 69, 074005 (2004).

[76] E. Braaten and M. Kusunoki, Phys. Rev. D 69, 114012 (2004).

[77] E. Braaten, M. Kusunoki and S. Nussinov, Phys. Rev. Lett. 93, 162001 (2004).

[78] E. Braaten and M. Kusunoki, Phys. Rev. D 71, 074005 (2005).

[79] E. Braaten and M. Kusunoki, Phys. Rev. D 72, 014012 (2005).

[80] E. Braaten and M. Kusunoki, Phys. Rev. D 72, 054022 (2005).

[81] M. T. Al Fiky, F. Gabbiani and A. A. Petrov, "X(3872): Hadronic molecules in effective field theory", arXiv:hep-ph/0506141

[82] A. A. Petrov, private communication.

[83] K. Abe et al. [Belle Collaboration], Phys. Rev. Lett. 93, 051803 (2004).

[84] B. Aubert et al. [BABAR Collaboration], arXiv:hep-ex/0510070

[85] J. Hilbert and E.S. Swanson, work in progress.

[86] M. Suzuki, Phys. Rev. D 72, 114013 (2005) arXiv:hep-ph/0508258.

[87] J. L. Rosner et al. [CLEO Collaboration], Phys. Rev. Lett. 95, 102003 (2005).

[88] S. Godfrey and J. L. Rosner, Phys. Rev. D 66, 014012 (2002) arXiv:hep-ph/0205255. See also Y. P. Kuang, Phys. Rev. D 65, 094024 (2002) arXiv:hep-ph/0201210.

[89] C. Edwards et al. [CBALL], Phys. Rev. Lett. 48, 70 (1982).

[90] S.-K. Choi et al. [Belle Collaboration], Phys. Rev. Lett. 89, 102001 (2002); P. Rubin et al. [CLEO Collaboration], Phys. Rev. D 72, 092004 (2005) arXiv:hep-ex/0508037.

[91] K. T. Chao, Y. F. Gu and S. F. Tuan, Commun. Theor. Phys. 25, 471 (1996).

[92] O. Lakhina and E. S. Swanson, arXiv:hep-ph/0603164 See also Z. Z. Song, C. Meng and K. T. Chao, Eur. Phys. J. C 
36, 365 (2004) arXiv:hep-ph/0209257.

[93] D.M. Asner et al. [CLEO Collaboration], Phys. Rev. Lett. 92, 142001 (2004).

[94] B. Aubert et al. [BABAR Collaboration], Phys. Rev. Lett. 92, 142002 (2004).

[95] E. J. Eichten, K. Lane and C. Quigg, Phys. Rev. D 69, 094019 (2004).

[96] K. Abe et al., arXiv:hep-ex/0507019

[97] But K. Trabelsi quotes a width of 39(26) $\mathrm{MeV}$ at Hadron05.

[98] S. Olsen, private communication.

[99] J. L. Rosner, "Hadron spectroscopy - a 2005 snapshot", arXiv:hep-ph/0508155

[100] K. Abe et al. [Belle Collaboration], Phys. Rev. D 70, 071102 (2004); P. Pakhlov [Belle Collaboration], arXiv:hep-ex/0412041

[101] E. Braaten and J. Lee, Phys. Rev. D. 67, 054007 (2003); see also G. T. Bodwin, J. Lee and E. Braaten, Phys. Rev. D 67, 054023 (2003) [Erratum-ibid. D 72, 099904 (2005)] arXiv:hep-ph/0212352.

[102] K. Y. Liu, Z. G. He and K. T. Chao, arXiv:hep-ph/0408141 K. Y. Liu, Z. G. He and K. T. Chao, Phys. Lett. B 557, 45 (2003) arXiv:hep-ph/0211181.

[103] A. E. Bondar and V. L. Chernyak, Phys. Lett. B 612, 215 (2005).

[104] V. V. Braguta, A. K. Likhoded and A. V. Luchinsky, Phys. Rev. D 72, 074019 (2005) arXiv:hep-ph/0507275.

[105] S.K. Choi et al. [Belle Collaboration], Phys. Rev. Lett. 94, 182002 (2005).

[106] N. Isgur, R. Kokoski and J. Paton, Phys. Rev. Lett. 54, 869 (1985); F. E. Close and P. R. Page, Nucl. Phys. B 443, 233 (1995); P. R. Page, E. S. Swanson and A. P. Szczepaniak, Phys. Rev. D 59, 034016 (1999).

[107] K. Abe et al., arXiv:hep-ex/0507033 S. Uehara et al. [Belle Collaboration], arXiv:hep-ex/0512035

[108] T. Barnes, Proc. IXth Intl. Conf. on Photon-Photon Collisions, (La Jolla, CA, 22-26 March 1992); T. Barnes, arXiv:hep-ph/0510365

[109] B. Aubert et al. [BABAR Collaboration], Phys. Rev. Lett. 95, 142001 (2005) arXiv:hep-ex/0506081.

[110] B. Aubert et al. [BABAR Collaboration], Phys. Rev. D73, 011101 (2006) arXiv:hep-ex/0507090.

[111] F. J. Llanes-Estrada, Phys. Rev. D 72, 031503 (2005).

[112] C. F. Qiao, arXiv:hep-ph/0510228

[113] L. Maiani, V. Riquer, F. Piccinini and A. D. Polosa, Phys. Rev. D 72, 031502 (2005).

[114] X. Liu, X. Q. Zeng and X. Q. Li, Phys. Rev. D 72, 054023 (2005)

[115] S. L. Zhu, Phys. Lett. B 625, 212 (2005);

[116] F. E. Close and P. R. Page, arXiv:hep-ph/0507199

[117] E. Kou and O. Pene, arXiv:hep-ph/0507119

[118] E. S. Swanson and A. P. Szczepaniak, Phys. Rev. D 59, 014035 (1999).

[119] C. McNeile, C. Michael and P. Pennanen [UKQCD Collaboration], Phys. Rev. D 65, 094505 (2002).

[120] T. W. Chiu and T. H. Hsieh [TWQCD Collaboration], arXiv:hep-lat/0512029

[121] B. Aubert et al. [BABAR Collaboration], Phys. Rev. Lett. 90, 242001 (2003);

[122] S. Godfrey and R. Kokoski, Phys. Rev. D43, 1679 (1991).

[123] There is a long and evolving history of the application of this idea. See, for example, A. DeRujula, H. Georgi, and S. Glashow, Phys. Rev. Lett. 37, 785 (1976); J. Rosner, C. Nucl. Part. Phys. 16, 109 (1986); N. Isgur and M. Wise, Phys. Rev. D45, 1553 (1992); and the previous reference.

[124] M.A. Nowak, M. Rho, and I. Zahed, Phys. Rev. D48, 4370 (1993).

[125] W.A. Bardeen and C.T. Hill, Phys. Rev. D49, 409 (1994).

[126] D. Ebert, T. Feldmann, R. Friedrich and H. Reinhardt, Nucl. Phys. B 434, 619 (1995) arXiv:hep-ph/9406220; D. Ebert, T. Feldmann and H. Reinhardt, Phys. Lett. B 388, 154 (1996) arXiv:hep-ph/9608223.

[127] W. A. Bardeen, E. J. Eichten and C. T. Hill, Phys. Rev. D 68, 054024 (2003); M. A. Nowak, M. Rho and I. Zahed, Acta Phys. Polon. B 35, 2377 (2004).

[128] T. D. Cohen and L. Y. Glozman, Phys. Rev. D 65, 016006 (2002); E. S. Swanson, Phys. Lett. B 582, 167 (2004); L. Y. Glozman, Phys. Lett. B 587, 69 (2004).

[129] N. Ligterink and E. S. Swanson, Phys. Rev. C 69, 025204 (2004) arXiv:hep-ph/0310070.

[130] R. L. Jaffe, D. Pirjol, and A. Scardicchio, "Why Massless Pions Preclude SU $(2)_{L} \times$ SU $(2)_{R}$ Restoration in the Hadron Spectrum", arXiv:hep-ph/0511081

[131] A review of charmed mesons is presented in P. Colangelo, F. De Fazio and R. Ferrandes, Mod. Phys. Lett. A 19, 2083 (2004) arXiv:hep-ph/0407137.

[132] D. Besson et al. [CLEO collaboration], Phys. Rev. D68, 032002 (2003).

[133] B. Aubert et al. [BABAR Collaboration], "Measurement of the D/sJ*(2317)+ and D/sJ(2460)+ properties in e+ e- $\rightarrow$ c anti-c production", arXiv:hep-ex/0408067

[134] B. Aubert et al. [BABAR Collaboration], Phys. Rev. D 69, 031101 (2004) arXiv:hep-ex/0310050.

[135] A. Drutskoy et al. [Belle collaboration], Phys. Rev. Lett. 94, 061802 (2004).

[136] K. Abe et al., "Improved measurements of anti-B0 $\rightarrow$ D/sJ+ K- decays", arXiv:hep-ex/0507064

[137] C.-H. Chen and H.-N. Li, Phys. Rev. D69, 054002 (2004).

[138] A. Drutskoy, Charm Hadronic Physics at Belle, HEP2005, Lisbon, July 21-27.

[139] P. Krokovny et al. [Belle Collaboration], Phys. Rev. Lett. 91, 262002 (2003) arXiv:hep-ex/0308019.

[140] P. Colangelo, F. De Fazio and A. Ozpineci, Phys. Rev. D 72, 074004 (2005) arXiv:hep-ph/0505195; P. Colangelo and F. De Fazio, Phys. Lett. B 570, 180 (2003) arXiv:hep-ph/0305140.

[141] R. Cahn and J.D. Jackson, Phys. Rev. D68, 037502 (2003). 
[142] J. Zeng, J. Van Orden, and W. Roberts, Phys. Rev. D52, 5229 (1995).

[143] S. Gupta and J. Johnson, Phys. Rev. D51, 168 (1995).

[144] D. Ebert, V. Galkin, and R. Faustov, Phys. Rev. D57, 5663 (1998).

[145] T. Lahde, C. Nyfalt, and D. Riska, Nucl. Phys. A674, 141 (2000).

[146] M. Di Pierro and E. Eichten, Phys. Rev. D64, 114004 (2001).

[147] T. Matsuki and T. Morii, Phys. Rev. D56, 5646 (1997).

[148] T. Matsuki, T. Morii and K. Sudoh, arXiv:hep-ph/0510269

[149] T. Matsuki, private communication.

[150] S. Godfrey, Phys. Lett. B 568, 254 (2003) arXiv:hep-ph/0305122.

[151] T. Barnes, F. E. Close and H. J. Lipkin, Phys. Rev. D 68, 054006 (2003) arXiv:hep-ph/0305025.

[152] X. Liu, Y. M. Yu, S. M. Zhao and X. Q. Li, arXiv:hep-ph/0601017

[153] A. P. Szczepaniak, Phys. Lett. B 567, 23 (2003) arXiv:hep-ph/0305060.

[154] D. S. Hwang and D. W. Kim, Phys. Lett. B 601, 137 (2004) arXiv:hep-ph/0408154. D.S. Hwang and D. W. Kim, Phys. Lett. B 606, 116 (2005) arXiv:hep-ph/0410301. D. S. Hwang and D. W. Kim, J. Phys. Conf. Ser. 9 (2005) 63.

[155] E. van Beveren and G. Rupp, Phys. Rev. Lett. 91, 012003 (2003) arXiv:hep-ph/0305035.

[156] K. Terasaki, Phys. Rev. D 68, 011501 (2003) arXiv:hep-ph/0305213; K. Terasaki, Phys. Rev. D59, 114001 (1999); A. Hayashigaki and K. Terasaki, "Isospin quantum number of D/s0(2317)+", arXiv:hep-ph/0410393 A. Hayashigaki and K. Terasaki, "Charmed-meson spectroscopy in QCD sum rule", arXiv:hep-ph/0411285.

[157] H. Y. Cheng and W. S. Hou, Phys. Lett. B 566, 193 (2003) arXiv:hep-ph/0305038.

[158] M. Nielsen, R. D. Matheus, F. S. Navarra, M. E. Bracco, and A. Lozea, "Diquark antidiquark with open charm in QCD sum rules", arXiv:hep-ph/0509131

[159] U. Dmitrasinovic, Phys. Rev. Lett. 94, 162002 (2005).

[160] H.J. Lipkin, Phys. Lett. 70B, 113 (1977).

[161] M. Suzuki and S.F. Tuan, Phys. Lett. 133B, 125 (1983).

[162] L. Maiani, F. Piccinini, A. D. Polosa and V. Riquer, Phys. Rev. Lett. 93, 212002 (2004) arXiv:hep-ph/0407017.

[163] Y. Q. Chen and X. Q. Li, Phys. Rev. Lett. 93, 232001 (2004) arXiv:hep-ph/0407062.

[164] S. Nussinov, "QCD inequalities and the D/s(2320)", arXiv:hep-ph/0306187

[165] T. E. Browder, S. Pakvasa, and A. A. Petrov, Phys. Lett. B 578, 365 (2004) arXiv:hep-ph/0307054.

[166] A. Dougall, R. D. Kenway, C. M. Maynard, and C. McNeile [UKQCD Collaboration], Phys. Lett. B 569, 41 (2003) arXiv:hep-lat/0307001.

[167] G. S. Bali, Phys. Rev. D 68, 071501 (2003) arXiv:hep-ph/0305209.

[168] A. V. Evdokimov et al. [SELEX Collaboration], Phys. Rev. Lett. 93, 242001 (2004) arXiv:hep-ex/0406045].

[169] T. Barnes, F. E. Close, J. J. Dudek, S. Godfrey and E. S. Swanson, Phys. Lett. B 600, 223 (2004) arXiv:hep-ph/0407120.

[170] L. Maiani, F. Piccinini, A.D. Polosa, and V. Riquer, Phys. Rev. D70, 054009 (2004).

[171] E. van Beveren and G. Rupp, Phys. Rev. Lett. 93, 202001 (2004) arXiv:hep-ph/0407281.

[172] K.-T. Chao, Phys. Lett. B599, 43 (2004).

[173] R. Kutschke, personal communication; E381-doc-701-v2.

[174] B. Aubert et al. [BABAR Collaboration], "Search for the D/sJ*(2632)+ at BaBar", arXiv:hep-ex/0408087

[175] R. Galik, talk presented at PIC2004.

[176] S. Godfrey, Phys. Rev. D 72, 054029 (2005) arXiv:hep-ph/0508078.

[177] T. Barnes, "HADRON05 summary: Heavy quark hadrons and theory", arXiv:hep-ph/0510365

[178] [DØ Collaboration], "Study of excited B mesons", D0 note 4517 (1 Aug 2004).

[179] F. Abe et al. [CDF Collaboration], Phys. Rev. Lett. 81, 2432 (1998); F. Abe et al. [CDF Collaboration], Phys. Rev. D58, 112004 (1998).

[180] D. Acosta et al. [CDF Collaboration], "Evidence for the exclusive decay B/c+- $\rightarrow \mathrm{J} / \mathrm{psi}$ pi+- and measurement of the mass of the B/c meson," arXiv:hep-ex/0505076

[181] M. Spezziga, "Studies of the Bc Meson at CDF," arXiv:hep-ex/0511007

[182] V. V. Kiselev, "Decays of the B/c meson", arXiv:hep-ph/0308214

[183] I. F. Allison, C. T. H. Davies, A. Gray, A. S. Kronfeld, P. B. Mackenzie and J. N. Simone [HPQCD Collaboration], Phys. Rev. Lett. 94, 172001 (2005) arXiv:hep-lat/0411027.

[184] S. Bianco, "Spectroscopy of D Mesons", hep-ex/0512073

[185] N. Isgur, "Hadron Spectroscopy: An Overview With Strings Attached", UTPT-89-13.

[186] T. E. O. Ericson and G. Karl, Phys. Lett. B 309, 426 (1993).

\section{APPENDIX A: BOUND STATE DECAY FORMALISM}

While the use of the weak binding decay relations of Sect. IID 1 are undoubtedly accurate for the $X(3872)$, it is useful to examine molecular decays in more depth to assess the accuracy of the weak binding approximation. Furthermore, it was assumed that the $\rho J / \psi$ decay to $\pi \pi J / \psi$ proceeded as if the $\rho J / \psi$ channel belonged in the bound state instead of in the continuum. This simplifying assumption will also be justified in the following.

We first consider the generic strong decay of a molecule, $X$ to a final state $\alpha \beta B$ via a bound state component, $A B$ illustrated in Fig. 39, In the ${ }^{3} P_{0}$ model this amplitude is 


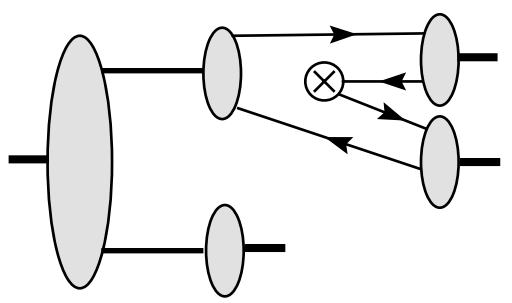

FIG. 39: Generic $X \rightarrow(A B) \rightarrow \alpha \beta B$ Decay.

$$
\mathcal{A}(X \rightarrow A B ; A \rightarrow \alpha \beta)=Z_{A B}^{1 / 2} \phi_{X(A B)}\left(q_{\alpha}+q_{\beta}\right) \int \frac{d^{3} k}{(2 \pi)^{3}} \phi_{A}(k) \phi_{\alpha}^{*}\left(k+\frac{q_{\beta}}{2}\right) \phi_{\beta}^{*}\left(k-\frac{q_{\alpha}}{2}\right)\langle\sigma\rangle \cdot\left(\vec{k}-\frac{\vec{q}_{\alpha}}{2}+\frac{\vec{q}_{\beta}}{2}\right)
$$

where $\langle\sigma\rangle$ is the matrix element of the Pauli matrices in the $A \alpha \beta$ spin states and $q_{\alpha}$ and $q_{\beta}$ are the momenta of the mesons $\alpha$ and $\beta$ respectively.

The differential decay rate is given by

$$
d \Gamma=|\mathcal{A}|^{2} d \phi_{3}=\left.Z_{A B}\left|\phi_{X(A B)}\left(q_{\alpha}+q_{\beta}\right)\right|^{2} \mathcal{A}(A \rightarrow \alpha \beta)\right|^{2}
$$

where $d \phi_{3}$ is the three particle phase space. Notice that the use of the ${ }^{3} P_{0}$ model is no longer relevant. Integrating and using the recursion property of phase space (Eq. 37.12 of Ref. [17]) gives

$$
\Gamma(X \rightarrow \alpha \beta B)=Z_{A B} \int \frac{d^{3} q}{(2 \pi)^{3}}\left|\phi_{X(A B)}(q)\right|^{2} \Gamma(A \rightarrow \alpha \beta)
$$

where the $A$ meson is taken to have energy $E_{A}=M(X)-E_{B}(q)$ and momentum $\vec{q}$. In the weak binding limit the $X$ wavefunction is strongly peaked at the origin and one obtains

$$
\Gamma(X \rightarrow \alpha \beta B) \approx Z_{A B} \Gamma(A \rightarrow \alpha \beta)
$$

where now $\Gamma(A \rightarrow \alpha \beta)$ is the experimentally determined free space partial width of the $A$. This identification requires $M(X)-M(B) \approx M(A)$ which is of course also true in the weak binding limit. Thus the weak binding formulas are justified for the case where the channel $A B$ is a bound state component of the molecule.

The use of the weak binding decay relation for $X \rightarrow \pi \pi J / \psi$ is more problematic because the $\rho J / \psi$ channel should be considered part of the continuum rather than part of the $X$ wavefunction as assumed in Sect. IID 1 Indeed, this process is second order in perturbation theory, as illustrated in Fig. 40 rather than first order as above. In this figure, the boxes represent intermediate states which are summed.

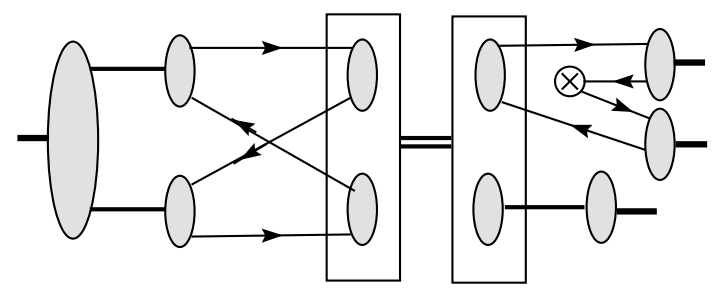

FIG. 40: Second Order Transition $X(A B) \rightarrow C D \rightarrow \alpha \beta D$.

The perturbative expression for the decay amplitude is

$$
\mathcal{A}(X(A B) \rightarrow C D \rightarrow \alpha \beta D)=\tilde{T}\left(q_{\alpha}+q_{\beta}\right) \frac{\mathcal{A}(B \rightarrow \alpha \beta))}{M(X)-E_{C D}+i \epsilon}
$$

where 


$$
\tilde{T}(q)=\int \frac{d^{3} p}{(2 \pi)^{3}} \phi_{X(A B)}(p) T_{A B \rightarrow C D}(p \rightarrow q) .
$$

and $T$ is the perturbative scattering matrix representing the subprocess $A B \rightarrow C D$ in Fig. 40,

Integrating over three body phase space yields the following expression for the width

$$
\Gamma(X \rightarrow \alpha \beta D)=\int \frac{d^{3} Q}{(2 \pi)^{3}} \Gamma(C \rightarrow \alpha \beta) \frac{|\tilde{T}(Q)|^{2}}{\left(M(X)-E_{C D}(Q)\right)^{2}+\Gamma_{C}^{2} / 4}
$$

where the total width of the $C$ meson has been restored to the propagator and it assumed that the $D$ meson is stable. The width for the $C$ is to be evaluated at the kinematical point $E_{C}=M(X)-E_{D}(Q)$.

In the limit where the $C$ particle is narrow this approaches

$$
\Gamma(X \rightarrow C D)=2 \pi Q \frac{E_{C} E_{D}}{M(X)} B r \int|\tilde{T}|^{2} d \Omega
$$

as expected. Furthermore, if the secondary amplitude $C \rightarrow \alpha \beta$ is independent of momentum Eq. A7 simplifies to

$$
\Gamma(X \rightarrow C D \rightarrow \alpha \beta D)=\Gamma(C \rightarrow \alpha \beta) \int \frac{|\tilde{T}|^{2}}{\left(M(X)-E_{C D}\right)^{2}}=Z_{C D} \Gamma(C \rightarrow \alpha \beta) .
$$

Thus the weak binding expression is recovered under the conditions that the production side of Fig. 40 and the decay side factorise. In general one expects no dependence of the $C$ width on the momentum of the $C$ so the constraint is certainly plausible.

\section{APPENDIX B: PION EXCHANGE POTENTIALS}

The standard quark-pion interaction (as used in nuclear physics) is given by

$$
L=-\frac{g}{\sqrt{2} f_{\pi}} \int d^{3} x \bar{\psi} \gamma_{\mu} \gamma_{5} \vec{\tau} \psi \cdot \partial^{\mu} \vec{\pi}
$$

where $f_{\pi}=92 \mathrm{MeV}$ and $g$ is the pion-quark coupling constant 61 . Effective pion exchange potentials are derived from the nonrelativistic limit of the appropriate $t$-channel scattering amplitude where the vertices are computed from the pion-quark interaction given above.

Extraction of the $\pi H H$ vertex is simplified by using the Hamiltonian 186 .

$$
H=i \sum_{q} \frac{g}{\sqrt{2} f_{\pi}} \vec{\sigma} \cdot \vec{k} \vec{\tau} \cdot \vec{\pi}
$$

where the sum refers to a sum over quark flavours. The computations may be simplified further by considering forward propagating neutral pions:

$$
H=i \sum_{q} \frac{g}{\sqrt{2} f_{\pi}} \sigma_{z} k \tau_{z} \pi^{0}
$$

For example, the effective one-pion-exchange induced NN interaction is obtained from the $\pi N N$ vertex which is given by

$$
\left\langle p_{\uparrow}|H| p_{\uparrow}\right\rangle=\frac{5}{3} i \frac{g}{\sqrt{2} f_{\pi}} k \pi^{0} .
$$


TABLE XV: One-pion-exchange $N N$ Interactions

\begin{tabular}{clcl}
\hline isospin & $J^{P}$ & channels & potential \\
\hline \hline 1 & $0^{+}$ & ${ }^{1} S_{0}$ & $-\frac{25}{3} V_{0} C(r)$ \\
1 & $0^{-}$ & ${ }^{3} P_{0}$ & $\frac{25}{9} V_{0}(C(r)-4 T(r))$ \\
0 & $1^{-}$ & ${ }^{1} P_{1}$ & $25 V_{0} C(r)$ \\
0 & $1^{+}{ }^{3} S_{1} /{ }^{3} D_{1}$ (the deuteron) & Eq. B11 \\
\hline \hline
\end{tabular}

This relation has been derived using the proton wavefunction

$$
\left|p_{\uparrow}\right\rangle=\frac{1}{\sqrt{6}}\left(\left|u_{\uparrow} u_{\downarrow} d_{\uparrow}\right\rangle+\left|u_{\downarrow} u_{\uparrow} d_{\uparrow}\right\rangle-2\left|u_{\uparrow} u_{\uparrow} d_{\downarrow}\right\rangle\right) .
$$

The NN scattering amplitude is then given by

$$
\mathcal{M}=-\frac{25}{9}\left(\frac{g}{\sqrt{2} f_{\pi}}\right)^{2} \vec{\tau}_{1} \cdot \vec{\tau}_{2} \frac{\sigma_{1} \cdot \vec{k} \sigma_{2} \cdot \vec{k}}{k^{2}+m_{\pi}^{2}}
$$

which yields the relationship $g_{N N \pi}=\frac{5}{3} \frac{g m_{N} \sqrt{2}}{f_{\pi}}$.

Fourier transforming gives the generic pion-induced interaction

$$
V_{\text {ope }}=\xi V_{0} \vec{\tau}_{1} \cdot \vec{\tau}_{2}\left[\sigma_{1} \cdot \sigma_{2} C(r)+\left(3 \sigma_{1} \cdot \hat{r} \vec{\sigma}_{2} \cdot \hat{r}-\sigma_{1} \cdot \sigma_{2}\right) T(r)\right]
$$

where $\xi$ is a numerical factor determined by the channel in question and

$$
\begin{gathered}
V_{0}=\frac{g^{2} m_{\pi}^{3}}{24 \pi f_{\pi}^{2}} \approx 1.3 \mathrm{MeV} \\
C(r)=\frac{\mathrm{e}^{-m_{\pi} r}}{m_{\pi} r}
\end{gathered}
$$

and

$$
T(r)=\left(\frac{3}{m_{\pi}^{2} r^{2}}+\frac{3}{m_{\pi} r}+1\right) C(r) .
$$

Nucleon-nucleon interactions may now be evaluated using $\xi=\frac{25}{9}$ and $\left\langle\vec{\tau}_{1} \cdot \vec{\tau}_{2}\right\rangle=2 I(I+1)-3$. Thus all possible $N N$ interactions are as listed in Table $\mathrm{XV}$

$$
V=-\frac{25}{3} V_{0}\left[\left(\begin{array}{ll}
1 & 0 \\
0 & 1
\end{array}\right) C(r)+\left(\begin{array}{cc}
0 & \sqrt{8} \\
\sqrt{8} & -2
\end{array}\right) T(r)\right]
$$

This corrects errors in Table 1 and Eq. 30 of Ref. 61.

The interaction is often regulated in an attempt to mimic unknown short range dynamics. It is traditional to do so with a dipole form factor such as $\left(\Lambda^{2}-m_{\pi}^{2}\right) /\left(\Lambda^{2}+t\right)$. The resulting softening of the potential is crucial to reproducing deuteron properties. In principle, the form factor can be computed by convoluting the spatial pion and nucleon wavefunctions. However, attempts to do so yield unsatisfactory results and the dipole form factor has been retained in $X$ the molecular computations reported here. Finally, we note that the form factor induces a very short range repulsive core (figures 1 and 2 of Ref. 61] are misleading) that, in part, explains the phenomenological usefulness of this method. The cutoff has the further benefit of providing a factorisation scale between long range pion exchange and short range quark exchange dynamics.

Computations for meson-meson interactions proceed in a similar way. For example vertices for $K$ or $K^{*}$ scattering are given by 
TABLE XVI: One-pion-exchange $M M$ Interactions

\begin{tabular}{|c|c|c|c|c|}
\hline state & isospin & $J^{P C}$ & channels & potential \\
\hline$D \bar{D}^{*}$ & 0 & $0^{-+}$ & ${ }^{3} P_{0}$ & $-3 V_{0}(C(r)+2 T(r))$ \\
\hline$D \bar{D}^{*}$ & 0 & $0^{--}$ & ${ }^{3} P_{0}$ & $+3 V_{0}(C(r)+2 T(r))$ \\
\hline$D \bar{D}^{*}$ & 0 & $1^{-+}$ & ${ }^{3} P_{1}$ & $-3 V_{0}(C(r)-T(r))$ \\
\hline$D \bar{D}^{*}$ & 0 & $1^{--}$ & ${ }^{3} P_{1}$ & $+3 V_{0}(C(r)-T(r))$ \\
\hline$D \bar{D}^{*}$ & 0 & $1^{++}$ & ${ }^{3} S_{1},{ }^{3} D_{1}$ & $+3 \cdot(\mathrm{Eq} \mathrm{B} 14$ \\
\hline$D \bar{D}^{*}$ & 0 & $1^{+-}$ & ${ }^{3} S_{1},{ }^{3} D_{1}$ & $-3 \cdot(\mathrm{Eq} \mathrm{B} 14$ \\
\hline$D D^{*}$ & 1 & $0^{-+}$ & ${ }^{3} P_{0}$ & $+V_{0}(C(r)+2 T(r))$ \\
\hline$D \bar{D}^{*}$ & 1 & $0^{--}$ & ${ }^{3} P_{0}$ & $-V_{0}(C(r)+2 T(r))$ \\
\hline$D \bar{D}^{*}$ & 1 & $1^{-+}$ & ${ }^{3} P_{1}$ & $+V_{0}(C(r)-T(r))$ \\
\hline$D \bar{D}^{*}$ & 1 & $1^{--}$ & ${ }^{3} P_{1}$ & $-V_{0}(C(r)-T(r))$ \\
\hline$D \bar{D}^{*}$ & 0 & $1^{++}$ & ${ }^{3} S_{1},{ }^{3} D_{1}$ & $-(\mathrm{Eq} \mathrm{B} 14)$ \\
\hline$D \bar{D}^{*}$ & 0 & $1^{+-}$ & ${ }^{3} S_{1},{ }^{3} D_{1}$ & $+(\mathrm{Eq} \mathrm{B14}$ \\
\hline$D^{*} \bar{D}^{*}$ & 0 & $0^{++}$ & ${ }^{\mathrm{T}} S_{0},{ }^{5} D_{0}$ & $6 \cdot(\mathrm{Eq} \mathrm{B} 15)$ \\
\hline$D^{*} \bar{D}^{*}$ & 0 & $0^{-+}$ & ${ }^{3} P_{0}$ & $-3 V_{0}(C(r)+2 T(r))$ \\
\hline$D^{*} \bar{D}^{*}$ & 0 & $1^{++}$ & ${ }^{5} D_{1}$ & $+3 V_{0}(C(r)-T(r))$ \\
\hline$D^{*} \bar{D}^{*}$ & 0 & $1^{-+}$ & ${ }^{3} P_{1}$ & $-3 V_{0}(C(r)-T(r))$ \\
\hline$D^{*} \bar{D}^{*}$ & 0 & $1^{+-}$ & ${ }^{3} S_{1},{ }^{3} D_{1}$ & $3 \cdot(\mathrm{Eq} \mathrm{B} 14)$ \\
\hline$D^{*} \bar{D}^{*}$ & 0 & $1^{--}$ & ${ }^{1} P_{1},{ }^{5} P_{1},{ }^{5} F_{1}$ & 6. (Eq $\overline{\mathrm{B} 16}$ \\
\hline$D^{*} \bar{D}^{*}$ & 0 & $2^{++}$ & ${ }^{1} D_{2},{ }^{5} S_{2},{ }^{5} D_{2},{ }^{5} G_{2}$ & 6. (Eq B17) \\
\hline$D^{*} \bar{D}^{*}$ & 0 & $2^{+-}$ & ${ }^{3} D_{2}$ & $-3 V_{0}(C(r)-T(r))$ \\
\hline$D^{*} \bar{D}^{*}$ & 1 & $0^{++}$ & ${ }^{1} S_{0},{ }^{5} D_{0}$ & $-2 \cdot(\mathrm{Eq} \mathrm{B} 15)$ \\
\hline$D^{*} \bar{D}^{*}$ & 1 & $0^{-+}$ & ${ }^{3} P_{0}$ & $+V_{0}(C(r)+2 T(r))$ \\
\hline$D^{*} \bar{D}^{*}$ & 1 & $1^{++}$ & ${ }^{5} D_{1}$ & $-V_{0}(C(r)-T(r))$ \\
\hline$D^{*} \bar{D}^{*}$ & 1 & $1^{-+}$ & ${ }^{3} P_{1}$ & $+V_{0}(C(r)-T(r))$ \\
\hline$D^{*} \bar{D}^{*}$ & 1 & $1^{+-}$ & ${ }^{3} S_{1},{ }^{3} D_{1}$ & $-(\mathrm{Eq} \mathrm{B} 14)$ \\
\hline$D^{*} \bar{D}^{*}$ & 1 & $1^{--}$ & ${ }^{1} P_{1},{ }^{5} P_{1},{ }^{5} F_{1}$ & $-2 \cdot(\mathrm{Eq} \mathrm{B} 16$ \\
\hline$D^{*} \bar{D}^{*}$ & 1 & $2^{++}$ & ${ }^{1} D_{2},{ }^{5} S_{2},{ }^{5} D_{2},{ }^{5} G_{2}$ & $-2 \cdot(\mathrm{Eq} \mathrm{B} 17$ \\
\hline$D^{*} \bar{D}^{*}$ & 1 & $2^{+-}$ & ${ }^{3} D_{2}$ & $V_{0}(C(r)-T(r))$ \\
\hline
\end{tabular}

$$
\langle K|H| K\rangle=\frac{g}{\sqrt{2} f_{\pi}} k \pi^{0}
$$

and

$$
\langle\bar{K}|H| \bar{K}\rangle=-\frac{g}{\sqrt{2} f_{\pi}} k \pi^{0} .
$$

Forming the $t$-channel scattering amplitude and Fourier transforming then yields the effective one-pion-exchange meson-meson interactions shown in Table XVI

$$
\begin{gathered}
V=-V_{0}\left[\left(\begin{array}{ll}
1 & 0 \\
0 & 1
\end{array}\right) C(r)+\left(\begin{array}{cc}
0 & -\sqrt{2} \\
-\sqrt{2} & 1
\end{array}\right) T(r)\right] . \\
V=-V_{0}\left[\left(\begin{array}{cc}
1 & 0 \\
0 & -\frac{1}{2}
\end{array}\right) C(r)+\left(\begin{array}{cc}
0 & \frac{1}{\sqrt{2}} \\
\frac{1}{\sqrt{2}} & 1
\end{array}\right) T(r)\right] . \\
V=-V_{0}\left[\left(\begin{array}{ccc}
1 & 0 & 0 \\
0 & -\frac{1}{2} & 0 \\
0 & 0 & -\frac{1}{2}
\end{array}\right) C(r)+\left(\begin{array}{ccc}
0 & \sqrt{\frac{4}{5}} & -\sqrt{\frac{6}{5}} \\
\sqrt{\frac{4}{5}} & -\frac{7}{5} & \sqrt{\frac{6}{25}} \\
-\sqrt{\frac{6}{5}} & \sqrt{\frac{6}{25}} & -\frac{8}{5}
\end{array}\right) T(r)\right]
\end{gathered}
$$




$$
\left.V=-V_{0}\left[\begin{array}{cccc}
1 & 0 & 0 & 0 \\
0 & -\frac{1}{2} & 0 & 0 \\
0 & 0 & -\frac{1}{2} & 0 \\
0 & 0 & 0 & -\frac{1}{2}
\end{array}\right) C(r)+\left(\begin{array}{cccc}
0 & \sqrt{\frac{1}{10}} & -\sqrt{\frac{1}{7}} & \sqrt{\frac{9}{35}} \\
\sqrt{\frac{1}{10}} & 0 & -\sqrt{\frac{7}{10}} & 0 \\
-\sqrt{\frac{1}{7}} & -\sqrt{\frac{7}{10}} & -\frac{3}{14} & -\frac{6 \sqrt{5}}{35} \\
\sqrt{\frac{9}{35}} & 0 & -\frac{6 \sqrt{5}}{35} & \frac{5}{7}
\end{array}\right) T(r)\right]
$$

